\title{
Mixed correlator dispersive CFT sum rules
}

\author{
Anh-Khoi Trinh \\ Department of Physics, McGill University, \\ 3600 Rue University, Montréal, QC H3A 2T8, Canada \\ E-mail: anh-khoi.trinh@mail.mcgill.ca
}

ABstract: Conformal field theory (CFT) dispersion relations reconstruct correlators in terms of their double discontinuity. When applied to the crossing equation, such dispersive transforms lead to sum rules that suppress the double-twist sector of the spectrum and enjoy positivity properties at large twist. In this paper, we construct dispersive CFT functionals for correlators of unequal scalar operators in position- and Mellin-space. We then evaluate these functionals in the Regge limit to construct mixed correlator holographic CFT functionals which probe scalar particle scattering in Anti-de Sitter spacetime. Finally, we test properties of these dispersive sum rules when applied to the 3D Ising model, and we use truncated sum rules to find approximate solutions to the crossing equation.

KEywords: AdS-CFT Correspondence, Conformal and W Symmetry, Conformal Field Theory

ArXiv EPrint: 2111.14731 


\section{Contents}

1 Introduction $\quad 2$

2 Overview of dispersive CFT sum rules 3

3 Position-space functionals 5

3.1 Generalities 5

3.2 Deriving the position-space $\mathfrak{B}_{\mathbf{k} \mid m n}^{\mathfrak{a}, \mathfrak{b}}$ kernel 7

$\begin{array}{ll}3.3 \text { Convergence and positivity properties: a plethora of subtraction schemes } & 12\end{array}$

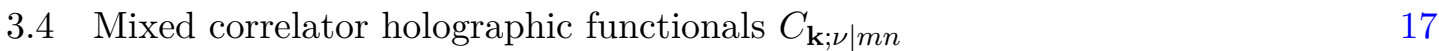

4 Mellin-space functionals $\quad 21$

4.1 Deriving the $\widehat{B}_{\mathbf{k} ; t \mid m n}^{s, t}$ functional 22

4.2 Mellin-space representation of holographic functionals 25

5 Applications to the 3D Ising model 26

5.1 Evaluating 3D Ising model sum rules 27

$\begin{array}{lll}5.1 .1 & \langle\sigma \sigma \sigma \sigma\rangle & 28\end{array}$

$\begin{array}{lll}5.1 .2\langle\epsilon \epsilon \epsilon\rangle & 29\end{array}$

$\begin{array}{lll}5.1 .3\langle\epsilon \sigma \epsilon \sigma\rangle & 31\end{array}$

5.1.4 $\langle\epsilon \sigma \sigma \epsilon\rangle \quad 33$

5.1.5 $\langle\sigma \sigma \epsilon \epsilon\rangle$ and $\langle\epsilon \epsilon \sigma \sigma\rangle \quad 34$

5.2 Approximate solutions to crossing from truncated sum rules 38

6 Discussion 40

A Detailed derivation of the $\mathfrak{B}_{\mathrm{k} \mid m n}^{\mathfrak{a}, \mathfrak{b}}$ kernel $\quad 42$

B $\mathfrak{B}_{\mathbf{k} \mid m n}^{\mathfrak{a}, \mathfrak{b}}$ kernel simplification $\quad 43$

C Mack polynomials $\quad 43$

D Mixed correlator analytic double-twist functionals $\quad 45$

$\begin{array}{lll}\mathrm{E} & \text { Varying } v \text { for the }\langle\sigma \sigma \sigma \sigma\rangle & \text { sum rule }\end{array}$ 


\section{Introduction}

The success of the modern conformal bootstrap stands upon the pillars of high precision numerical methods $[1,2]$, and deeper understanding of the analytic structure of the bootstrap equations (see $[3,4]$ and references therein). Numerical methods have led to high precision measurements of critical exponents of conformal field theories (CFTs) such as the 3D Ising [5-8] and the $O(N)$ models [9-11], while analytical methods have proven essential to the study of quantum gravity in Anti-de Sitter (AdS) spacetime [12-18] and perturbative CFTs $[19,20]$. In recent years, with the introduction of analytic functionals [21-28] and dispersion relation methods [29-35], the gap between numerics and analytics has greatly narrowed revealing new horizons for the bootstrap program. This paper builds upon this bridge by introducing new analytic dispersive functionals for correlators with unequal external scalar operators.

The numerical bootstrap relies upon a set of linear functionals that act on the crossing equation to determine whether crossing can be satisfied. The derivative functional has long served as the cornerstone for the numerical bootstrap, but progress in the field has encouraged the pursuit of new methods with more desirable positivity properties and computational efficiency. A potential candidate with such features is a so-called dispersive CFT functional first introduced in [30], which reconstructs functions in terms of their absorptive part [29]. Such a functional can be written as a dispersive transform acting on a stripped correlator $\mathcal{G}(u, v)$ :

$$
\mathcal{G}(u, v)=\mathcal{G}^{s}(u, v)+\mathcal{G}^{t}(u, v), \quad \mathcal{G}^{s, t}(u, v)=\iint d u^{\prime} d v^{\prime} K\left(u, v ; u^{\prime}, v^{\prime}\right) \operatorname{dDisc}_{s, t}\left[\mathcal{G}\left(u^{\prime}, v^{\prime}\right)\right]
$$

where we define the double discontinuity $\operatorname{dDisc}\left[\mathcal{G}\left(u^{\prime}, v^{\prime}\right)\right]$ in section 3.1 , and the cross-ratio variables are

$$
\begin{aligned}
u & =z \bar{z}, \quad v=(1-z)(1-\bar{z}), \\
u^{\prime} & =w \bar{w}, \quad v^{\prime}=(1-w)(1-\bar{w}) .
\end{aligned}
$$

In this example, $\mathcal{G}^{s, t}(u, v)$ can be thought as a functional acting on $\mathcal{G}(u, v){ }^{1}$

For equal scalar operators of scaling dimension $\Delta_{\phi}$, the authors in [30] introduced the collinear $B_{k, v}$ dispersive functional which enjoys the following three key properties:

- they commute with the OPE,

- they are sign definite above a twist threshold $\tau \geq 2 \Delta_{\phi}+k-2$, where $k \in 2 \mathbb{Z}$,

- and they return double-zeros when acting on double-twist operators.

The first property is referred to as swappability, and when combined with the second property, they lead to sum rules which balance the contribution of low twist operators to that of heavy operators:

$$
\sum_{\mathcal{O}_{\text {light }}} a_{\mathcal{O}_{\text {light }}} w[\mathcal{G}]+\sum_{\mathcal{O}_{\text {heavy }}} a_{\mathcal{O}_{\text {heavy }}} w[\mathcal{G}]=0
$$

\footnotetext{
${ }^{1}$ We will refer to these transforms interchangeably as functionals and dispersive transforms.
} 
where $a_{\mathcal{O}}=f_{12 \mathcal{O}} f_{43 \mathcal{O}}$ is the OPE coefficient square, and $w[\cdot]$ is some dispersive functional. If the set of heavy operators consists mostly of double-twist operators, the last property suppresses their contribution to the sum rule effectively allowing one to probe non-perturbative properties of CFTs. Together, these features represent an enticing addition to the CFT bootstrap toolkit.

When evaluated in the Regge limit, these dispersive functionals are further capable of probing bulk AdS physics. This insight led to the construction of holographic functionals in [31] where the collinear $B_{k, v}$ functional served as a seed in their construction. Such holographic functionals are capable of bounding couplings in AdS, and they become dispersion relations in the flat-space limit [36, 37].

Unfortunately, evaluating these $B_{k, v}$ functionals in position-space can become expensive in certain limits such as for large number of derivatives, or for low twist operators (near the unitarity bound). An alternative approach is to construct them in Mellin-space [38-42] where they can be computationally more efficient, and where projected functionals such as the $\Phi_{\ell}$ functional introduced in [30] are easier to construct. This paper builds upon the construction of dispersion relations in both position- and Mellin-space by extending previous results to mixed correlators.

In section 2, we briefly review the fundamental features of dispersive CFT sum rules before constructing the position-space kernel $B_{\mathbf{k} ; v \mid m n}^{s, t}$ in section 3 , where we further expound on their convergence and positivity properties. We then use our results to derive holographic dispersive functionals for mixed correlators in section 3.4. In section 4, we perform a corresponding analysis in Mellin-space which benefits from improved computational efficiency and manifest zero structure. Finally, in section 5, we apply these functionals to study the 3D Ising model. We first evaluate sum rules using our functionals on mixed correlators to verify their convergence and precision properties. We then use insight gained from this analysis to build a system of truncated sum rules to estimate approximate solutions to crossing for the 3D Ising model.

\section{Overview of dispersive CFT sum rules}

Dispersive transforms repackage information encoded in the usual OPE and crossing equations into dispersive sum rules:

$$
\begin{aligned}
& \sum_{\mathcal{O}_{s}} f_{\phi_{1} \phi_{2} \mathcal{O}_{s}} f_{\phi_{4} \phi_{3} \mathcal{O}_{s}} G_{\Delta_{\mathcal{O}_{s}}, J_{\mathcal{O}_{s}}}^{s}=\sum_{\mathcal{O}_{t}} f_{\phi_{3} \phi_{2} \mathcal{O}_{t}} f_{\phi_{4} \phi_{1} \mathcal{O}_{t}} G_{\Delta_{\mathcal{O}_{t}}, J_{\mathcal{O}_{t}}}^{t} \\
& \rightarrow \sum_{\mathcal{O}_{s}} f_{\phi_{1} \phi_{2} \mathcal{O}_{s}} f_{\phi_{4} \phi_{3} \mathcal{O}_{s}} w\left[G_{\Delta_{\mathcal{O}_{s}}, J_{\mathcal{O}_{s}}}^{s}\right]-\sum_{\mathcal{O}_{t}} f_{\phi_{3} \phi_{2} \mathcal{O}_{t}} f_{\phi_{4} \phi_{1} \mathcal{O}_{t}} w\left[G_{\Delta_{\mathcal{O}_{t}}, J_{\mathcal{O}_{t}}}^{t}\right]=0
\end{aligned}
$$

where $f_{i j k}$ are OPE coefficients, $G_{\Delta, J}^{s, t}$ are $s$ - and $t$-channel blocks respectively defined at the beginning of the next section, and $w[\cdot]$ is a dispersive functional with the key property that the action on double-twist operators is suppressed. Swappability ensures that the action of the functional commutes with the OPE. We will briefly sketch how to obtain dispersive sum rules in this section. 
A transform is said to be dispersive if it reconstructs the correlator in terms of its absorptive part. For CFTs, the latter corresponds to the double discontinuity of the correlator. Therefore, by construction, we seek the following position-space dispersive transform:

$$
\mathcal{G}^{s, t}(u, v)=\iint d u^{\prime} d v^{\prime} K\left(u, v ; u^{\prime}, v^{\prime}\right) \operatorname{dDisc}_{s, t}\left[\mathcal{G}\left(u^{\prime}, v^{\prime}\right)\right],
$$

where $\mathcal{G}(u, v)$ is the stripped correlator corresponding to eq. (3.1). We aim to derive kernels $K\left(u, v ; u^{\prime}, v^{\prime}\right)$ valid for any CFT correlator. To achieve this, one must ensure that such transforms are convergent, in particular in the Regge limit (see section 3.3 for an extended discussion). Convergence in the Regge limit is best understood in Mellin-space.

Our dispersive functionals are inspired by boundedness of the Mellin amplitude:

$$
\oint \frac{d \mathrm{~s}^{\prime}}{2 \pi i} \frac{M(\mathrm{~s}, \mathrm{t})}{\mathrm{s}-\mathrm{s}^{\prime}}=0
$$

where s, t are Mellin-mandelstam variables. Boundedness as s, t $\rightarrow i \infty$ corresponds to boundedness in the (u-channel) Regge limit. As shown in [32, 43], Mellin amplitudes are bounded as follows:

$$
\lim _{|\mathrm{s}| \rightarrow \infty}|M(\mathrm{~s}, \mathrm{t})|_{\mathrm{s}+\mathrm{t} \text { fixed }}=|\mathrm{s}|^{J},
$$

where $J=1$ for physical correlators. Therefore, Regge bounded Mellin amplitudes can be obtained by an appropriate rescaling:

$$
M(\mathrm{~s}, \mathrm{t}) \rightarrow \frac{M(\mathrm{~s}, \mathrm{t})}{\prod_{m, n=0}^{m_{\max , n_{\max }}\left(\mathrm{s}-\mathrm{s}_{m}\right)\left(\mathrm{t}-\mathrm{t}_{n}\right)}}
$$

These rescaled amplitudes lead to spin- $k$ subtracted dispersion relations denoted by subscripts $k$ where $k=n_{\max }+m_{\max }$. By performing an inverse Mellin transform, one can obtain a position-space representation of these subtracted dispersion relations valid for any spin- $k$ bounded CFT correlators (by following the nomenclature of eq. (2.4)):

$$
\mathcal{G}_{k}^{s, t}(u, v)=\iint d u^{\prime} d v^{\prime} K_{k}\left(u, v ; u^{\prime}, v^{\prime}\right) \operatorname{dDisc}_{s, t}\left[\mathcal{G}_{k}\left(u^{\prime}, v^{\prime}\right)\right] .
$$

If one applies these dispersive transforms on individual conformal blocks, the result is a so-called Polyakov-Regge block denoted as

$$
\mathcal{P}_{k ; \Delta, J}^{s, t}(u, v)=\iint d u^{\prime} d v^{\prime} K_{k}\left(u, v ; u^{\prime}, v^{\prime}\right) \operatorname{dDisc}_{s, t}\left[G_{\Delta, J}^{s, t}\left(u^{\prime}, v^{\prime}\right)\right] .
$$

By expanding the OPE in eq. (2.1), one concludes that such Regge-bounded correlators can be written in terms of a basis of $s$ - and $t$-channel Polyakov-Regge blocks:

$$
\mathcal{G}_{k}(u, v)=\sum_{\mathcal{O}_{s}} f_{12 \mathcal{O}_{s}} f_{43 \mathcal{O}_{s}} \mathcal{P}_{k ; \Delta_{\mathcal{O}_{s}}, J_{\mathcal{O}_{s}}}^{s}(u, v)+\sum_{\mathcal{O}_{t}} f_{32 \mathcal{O}_{t}} f_{41 \mathcal{O}_{t}} \mathcal{P}_{k ; \Delta_{\mathcal{O}_{t}}, J_{\mathcal{O}_{t}}}^{t}(u, v)
$$

The above is an example of a dispersive sum rule.

While the dispersion relation above allows one to fully reconstruct a correlator from its absorptive part, our goal is to constrain CFT data using the least number of assumptions. 
Therefore, one can obtain simpler dispersion relation by taking the $s$-channel collinear limit $u \rightarrow 0$ of these Polyakov-Regge blocks:

$$
\mathcal{P}_{k ; \Delta, J}^{s, t}(u, v) \underset{u \rightarrow 0}{\rightarrow} B_{k ; v \mid m n}^{s, t} .
$$

For equal external operators, there is a unique collinear expansion. This is no longer true for mixed correlators where the collinear limit allows for two $u$ expansions:

$$
\mathcal{P}(u, v)=\left(u^{\frac{\Delta_{3}+\Delta_{4}-\Delta_{1}-\Delta_{2}}{2}}+\ldots\right) f(v)+\left(u^{0}+\ldots\right) g(v)
$$

where the $\Delta_{i}$ dependence is fixed by our convention given in eq. (3.1). The ellipses denote subleading powers of $u$. We identify the expansions of $u$ by their positive $\Delta_{i}$ powers i.e. $(m n)=\{(34),(12)\}$ corresponding to $u \frac{\frac{\Delta_{m}+\Delta_{n}-\Delta_{1}-\Delta_{2}}{2}}{2}$.

Taking the $s$-channel collinear limit leads to a new family of functionals labelled by $(m n)$ and $v$ with their own kernels:

$$
B_{\mathbf{k} ; v \mid m n}^{s, t}=\iint d u^{\prime} d v^{\prime} \mathfrak{B}_{\mathbf{k} \mid m n}^{\mathfrak{a}, \mathfrak{b}}\left(v ; u^{\prime}, v^{\prime}\right) \operatorname{dDisc}_{s, t}\left[G_{\Delta, J}^{s, t}\left(u^{\prime}, v^{\prime}\right)\right] .
$$

We will show that these dispersive collinear functionals lead to the following sum rule:

$$
\sum_{\mathcal{O}_{s}} f_{12 \mathcal{O}_{s}} f_{43 \mathcal{O}_{s}} B_{\mathbf{k} ; v \mid m n}^{s}+\sum_{\mathcal{O}_{t}} f_{32 \mathcal{O}_{t}} f_{41 \mathcal{O}_{t}} B_{\mathbf{k} ; v \mid m n}^{t}=\mathbf{1}_{\mathrm{u}},
$$

where $\mathbf{1}_{\mathrm{u}}=1$ is the $\mathrm{u}$-channel identity. ${ }^{2}$ The derivation and analysis of these collinear $B_{\mathbf{k} ; v \mid 34}$ functionals are the main goals of this paper.

As mentioned in the introduction, these dispersive functionals enjoy appealing features. Firstly, these functionals are constructed in terms of their double discontinuity rather than the full correlator. Moreover, the presence of the double discontinuity suppresses doubletwist operators thereby allowing us to probe non-perturbative features of CFTs. Finally, they enjoy positivity properties due to the double-zeros that make them desirable for the numerical bootstrap. That being said, from eq. (2.12), it is apparent that positivity properties can no longer be exploited as straightforwardly for mixed correlators: individually, the $s$ - and $t$-channel functionals enjoy sign definiteness, however the sum does not. We will verify these statements in this paper.

\section{Position-space functionals}

\subsection{Generalities}

We consider the four-point function of unequal scalar operators with scaling dimensions $\Delta_{i}$ written as

$$
\left\langle\phi_{1}\left(x_{1}\right) \phi_{2}\left(x_{2}\right) \phi_{3}\left(x_{3}\right) \phi_{4}\left(x_{4}\right)\right\rangle=\frac{1}{\left(x_{13}^{2}\right)^{\frac{\Delta_{1}+\Delta_{2}+\Delta_{3}-\Delta_{4}}{2}}\left(x_{24}^{2}\right)^{\Delta_{2}}} \frac{\left(x_{34}^{2}\right)^{\frac{\Delta_{1}+\Delta_{2}-\Delta_{3}-\Delta_{4}}{2}}}{\left(x_{14}^{2}\right)^{\frac{\Delta_{1}+\Delta_{4}-\Delta_{2}-\Delta_{3}}{2}}} \mathcal{G}(u, v) .
$$

\footnotetext{
${ }^{2}$ Our convention differs from that of [30, 31]: for equal operators, the u-channel identity is always present. Therefore, they define their sum rules to be normalized as follows:

$$
\sum_{\mathcal{O}} f_{\phi \phi \mathcal{O}}^{2} B_{k, v}\left[G_{\Delta_{\mathcal{O}}, J_{\mathcal{O}}}^{s}\right]=(-1)^{k / 2-1}
$$


where $u, v$ correspond to the usual cross-ratios

$$
u=z \bar{z}=\frac{x_{12}^{2} x_{34}^{2}}{x_{13}^{2} x_{24}^{2}}, \quad v=(1-z)(1-\bar{z})=\frac{x_{14}^{2} x_{23}^{2}}{x_{13}^{2} x_{24}^{2}} .
$$

This unconventional prefactor is chosen to fix the u-channel identity to be $u^{-\frac{\Delta_{2}-\Delta_{4}}{2}}$; we will highlight certain key distinctions in the following paragraphs. This stripped prefactor fixes the normalization of the $s$ - and $t$-channel conformal blocks as follows:

$$
\begin{aligned}
& G_{\Delta, J}^{s ; \Delta_{1} \Delta_{2} \Delta_{3} \Delta_{4}}(z, \bar{z}) \sim(z \bar{z})^{-\frac{\Delta_{1}+\Delta_{2}}{2}} \times z^{\frac{\Delta-J}{2}} \bar{z}^{\frac{\Delta+J}{2}}, \quad \text { for } 0<z \ll \bar{z} \ll 1, \\
& G_{\Delta, J}^{t ; \Delta_{1} \Delta_{2} \Delta_{3} \Delta_{4}}(z, \bar{z})=G_{\Delta, J}^{s ; \Delta_{3} \Delta_{2} \Delta_{1} \Delta_{4}}(1-z, 1-\bar{z}) .
\end{aligned}
$$

We will abstain from writing the superscripts $\Delta_{i}$ in the future and refer to the definitions above for the $s$ - and $t$-channel conformal blocks $G_{\Delta, J}^{s}$ and $G_{\Delta, J}^{t}$. The blocks are further parametrized by the difference in scaling dimensions

$$
a=\frac{\Delta_{21}}{2}, \quad b=\frac{\Delta_{34}}{2},
$$

where $\Delta_{i j}=\Delta_{i}-\Delta_{j}$.

Let us record the behaviour of conformal blocks in the u-channel Regge limit. The Regge limit is reached by taking $x_{1} \rightarrow x_{3}$ and $x_{2} \rightarrow x_{4}$ along the lightcone; in terms of the cross-ratio variables, we take $z, \bar{z} \rightarrow+i \infty$ while fixing $z / \bar{z}$. In this limit, we find

$$
\begin{aligned}
G_{\Delta, J}^{s}(z, \bar{z}), G_{\Delta, J}^{t}(z, \bar{z}) & \sim z^{-2 \Delta_{2}}+z^{-\left(\Delta_{1}+\Delta_{2}+\Delta_{3}-\Delta_{4}\right)}+z^{-\frac{\Delta_{1}+3 \Delta_{2}+\Delta_{3}-\Delta_{4}}{2}} \\
G_{\Delta, J}^{u}(z, \bar{z}) & \sim z^{-\Delta_{2}+\Delta_{4}+j-1}
\end{aligned}
$$

Notably, $s$ - and $t$-channel conformal blocks are more convergent in the Regge limit than their u-channel counterpart for arbitrary spin $J$. We present these limits to highlight the overall $\Delta_{i}$ dependence for mixed correlators which will play an important role in studying convergence properties of our dispersive transforms.

Finally, given our convention, the crossing relations now reads

$$
\begin{array}{ll}
\mathcal{G}(z, \bar{z})=\mathcal{G}^{\Delta_{1} \leftrightarrow \Delta_{3}}(1-z, 1-\bar{z}) & (\mathrm{s} \leftrightarrow \mathrm{t}) \\
\mathcal{G}(z, \bar{z})=(z \bar{z})^{\Delta_{2}} \mathcal{G}^{\Delta_{1} \leftrightarrow \Delta_{4}}(1 / z, 1 / \bar{z}) & (\mathrm{s} \leftrightarrow \mathrm{u}), \\
\mathcal{G}(z, \bar{z})=((1-z)(1-\bar{z}))^{\frac{\Delta_{1}+\Delta_{2}+\Delta_{3}-\Delta_{4}}{2}} \mathcal{G}^{\Delta_{1} \leftrightarrow \Delta_{2}}\left(\frac{z}{1-z}, \frac{\bar{z}}{1-\bar{z}}\right) & (\mathrm{t} \leftrightarrow \mathrm{u})
\end{array}
$$

The stripped prefactor in eq. (3.1) was chosen given the desired properties of our dispersive functionals. In particular, the latter exhibit manifest crossing symmetry by construction since they are derived in the crossing region $\mathcal{R} \times \mathcal{R}$, where $\mathcal{R}=\mathbb{C} \backslash((-\infty, 0] \cup[1, \infty))$ is the cut plane; this is further illustrated by the lack of overall $z, \bar{z}$ factors in eq. (3.6a). In this domain, the $s$-channel OPE converges in $z \in \mathbb{C} \backslash[1, \infty)$, while the $t$-channel OPE converges in $\mathbb{C} \backslash(-\infty, 0]$. The domain of convergence for both $s$ - and $t$-channels are a subset of the Lorentzian lightcones $L_{u s}$ and $L_{t u}$ respectively, where the notation highlights the channels where the OPE converges; this notation is further elaborated in section 3.3 
where we discuss the integration region of these dispersive transforms. We underscore the fact that these domains encode information about the u-channel OPE.

For a two-variable function $G(w, \bar{w})$ defined in the crossing region, we define the double discontinuity as follows:

$$
\begin{aligned}
\operatorname{dDisc}_{s}[G(w, \bar{w})] & =\frac{1}{2}\left(G\left(w_{+}, \bar{w}_{-}\right)+G\left(w_{-}, \bar{w}_{+}\right)-G\left(w_{-}, \bar{w}_{-}\right)-G\left(w_{+}, \bar{w}_{+}\right)\right) \text {for } w, \bar{w}<0 \\
\operatorname{dDisc}_{t}[G(w, \bar{w})] & =\frac{1}{2}\left(G\left(w_{+}, \bar{w}_{-}\right)+G\left(w_{-}, \bar{w}_{+}\right)-G\left(w_{-}, \bar{w}_{-}\right)-G\left(w_{+}, \bar{w}_{+}\right)\right) \text {for } w, \bar{w}>1 .
\end{aligned}
$$

When $G(w, \bar{w})$ is the stripped correlator $\mathcal{G}(w, \bar{w})$ on the r.h.s. of eq. (3.1), one can show that for $w, \bar{w}<0$, the above reduces to the usual definition of the double discontinuity used in the Lorentzian inversion formula [44]:

$$
\begin{aligned}
\operatorname{dDisc}_{s} G(w, \bar{w}) \equiv & \cos (\pi(a+b)) G_{E}(w, \bar{w}) \\
& -\frac{e^{i \pi(a+b)}}{2} G_{E}(w, \bar{w} \circlearrowright 0)-\frac{e^{-i \pi(a+b)}}{2} G_{E}(w, \bar{w} \circlearrowleft 0) .
\end{aligned}
$$

A similar relation exists for the double discontinuity of the $t$-channel when $w, \bar{w}>1$. The relations above are made possible because the stripped correlator is Euclidean singlevalued $[16,26]{ }^{3}$ which assigns a Lorentzian interpretation to the discontinuities in the crossing region as originating from the double commutator of four operators in Lorentzian kinematics. The latter can be interpreted as a scattering matrix where pairs of operators are time-like from each other in different Rindler wedges. This interpretation makes it clear that it is a positive definite matrix with respect to the two pairs 12 and 34 .

Indeed, when acting on a conformal block, the double discontinuity multiplies the blocks by sine functions:

$$
\operatorname{dDisc}_{s}\left[G_{\Delta, J}^{s}(w, \bar{w})\right]=2 \sin \left(\frac{\Delta-J-\Delta_{1}-\Delta_{2}}{2} \pi\right) \sin \left(\frac{\Delta-J-\Delta_{3}-\Delta_{4}}{2} \pi\right) G_{\Delta, J}^{s}(w, \bar{w}) .
$$

An analogous relation exists for the double discontinuity across the $t$-channel cut. Crucially, if $\Delta_{1}+\Delta_{2}-\Delta_{3}-\Delta_{4} \in 2 \mathbb{Z}$, the $s$-channel double discontinuity returns double-zeros when $\Delta$ takes on $s$-channel double-twist values $\Delta_{n, J}=\Delta_{1}+\Delta_{2}+J+2 n$ thereby suppressing the double-twist sector of the spectrum, and ensuring that the double discontinuity is positive definite. This feature plays a key role in probing non-perturbative features of CFTs. For mixed correlators, simple zeros are allowed which causes the double discontinuity to no longer be positive definite.

\subsection{Deriving the position-space $\mathfrak{B}_{\mathbf{k} \mid m n}^{\mathfrak{a}, \mathfrak{b}}$ kernel}

After detailing our convention, we are now ready to derive the dispersion kernel associated with the collinear functional $B_{\mathbf{k} ; v}^{s, t}$. As discussed in the previous section, these functionals are defined as the coefficient of the $u \rightarrow 0$ power of the full dispersion kernel in

\footnotetext{
${ }^{3}$ Given a branch point at $(w, \bar{w})=(p, p)$, a function is Euclidean single-valued if and only if

$$
G\left(w_{+}, \bar{w}_{-}\right)=G\left(w_{-}, \bar{w}_{+}\right), \quad w_{ \pm}=w \pm i 0 .
$$
}

For the remainder of this paper, Euclidean single-valued objects are simply referred to as being single-valued. 
eq. (1.1). For generic external scalars, the small $u$ limit leads to two expansions as shown in eq. (2.10). We label the functionals by pairs of integers $(m n) \in\{(34),(12)\}$ associated with the corresponding double-twist family. Subleading $s$-channel double-twist trajectories can be measured by virtue of the soon-to-be presented subtraction schemes.

We seek to compute the kernel associated with eq. (2.11) which we rewrite here for convenience:

$$
B_{\mathbf{k} ; v \mid m n}^{s, t}=\iint d u^{\prime} d v^{\prime} \mathfrak{B}_{\mathbf{k} \mid m n}^{\mathfrak{a}, \mathfrak{b}}\left(v ; u^{\prime}, v^{\prime}\right) \operatorname{dDisc}_{s, t}\left[G_{\Delta, J}^{s, t}\left(u^{\prime}, v^{\prime}\right)\right],
$$

where the labels will be defined later near eq. (3.18). To achieve this goal, we will proceed by starting with a subtracted Mellin-space dispersion relation.

As discussed in the previous section, boundedness of the correlator in the u-channel Regge limit is necessary to obtain convergent dispersive sum rules; this in turn translates into the derivation of spin- $k$ bounded kernels. To derive such a kernel, the authors of [30] proposed to apply Cauchy's formula to a rescaled crossing-symmetric Mellin amplitude as shown by eq. (2.5) where the $\mathrm{s}$ and $\mathrm{t}$ poles are located at $s$ - and $t$-channel double-twist values. For equal external operators, there is a single double-twist family $\tau=2 \Delta_{\phi}-\ell+2 m$ for both $s$ - and $t$-channels. For generic correlators, we are free to subtract operators with different double-twist families $\tau_{i j}=\Delta_{i}+\Delta_{j}-\ell+2 m$. Therefore, we promote the spin- $k$ convergence label to a vector $k \rightarrow \mathbf{k}=\left(k_{12}, k_{23}, k_{34}, k_{14}\right)$ where the vector components $k_{i j}$ are associated with the double-twist pairs $\tau_{i j}$ and the sum of the vector components determines the u-channel Regge decay rate of the kernel. This can be understood in Mellin space as shown in eq. (2.5).

We therefore define the following ratios of Pochhammer symbols which will allow us to implement various subtractions schemes:

$$
P_{\mathbf{k}}\left(\mathrm{s}, \mathrm{t} ; \mathrm{s}^{\prime}, \mathrm{t}^{\prime}\right)=\frac{\left(\frac{\Delta_{1}+\Delta_{2}-\mathrm{s}}{2}\right)_{k_{12}}\left(\frac{\Delta_{2}+\Delta_{3}-\mathrm{t}}{2}\right)_{k_{23}}\left(\frac{\Delta_{3}+\Delta_{4}-\mathrm{s}}{2}\right)_{k_{34}}\left(\frac{\Delta_{1}+\Delta_{4}-\mathrm{t}}{2}\right)_{k_{14}}}{\left(\frac{\Delta_{1}+\Delta_{2}-\mathrm{s}^{\prime}}{2}\right)_{k_{12}}\left(\frac{\Delta_{2}+\Delta_{3}-\mathrm{t}^{\prime}}{2}\right)_{k_{23}}\left(\frac{\Delta_{3}+\Delta_{4}-\mathrm{s}^{\prime}}{2}\right)_{k_{34}}\left(\frac{\Delta_{1}+\Delta_{4}-\mathrm{t}^{\prime}}{2}\right)_{k_{14}}}
$$

Zeros in $\mathrm{s}$ and $\mathrm{t}$ cancel poles from the Mellin amplitude, while poles in $\mathrm{s}^{\prime}, \mathrm{t}^{\prime}$ strengthens the convergence in the Regge limit.

By construction, $k_{i j} \in \mathbb{Z} .{ }^{4}$ However, the range of $k_{i j}$ can be further restricted depending on the scaling dimensions of the external operators; this is detailled in section 3.3 when we discuss convergence properties. Between the two collinear expansion towers in $u$ and the various subtraction combinations labelled by $\mathbf{k}$, it might appear that the space of dispersive sum rules has greatly expanded. However, we will find relations between different subtraction schemes and operator ordering.

We now derive the $\mathfrak{B}_{\mathbf{k} \mid m n}^{\mathfrak{a}, \mathfrak{b}}$ dispersion kernel for mixed correlators in position-space based on the Mellin-inspired dispersion relation of [30]. The Mellin-space dispersion relation can be written as a contour integral in the s'-plane as follows:

$$
0=\oint \frac{d s^{\prime}}{2 \pi i} \frac{M_{\mathrm{k}}\left(\mathrm{s}, \mathrm{t} ; \mathrm{s}^{\prime}, \mathrm{t}^{\prime}\right)}{\mathrm{s}-\mathrm{s}^{\prime}}=M_{\mathrm{k}}^{s}\left(\mathrm{~s}, \mathrm{t} ; \mathrm{s}, \mathrm{t}^{\prime}\right)+M_{\mathbf{k}}^{t}\left(\mathrm{~s}, \mathrm{t} ; \mathrm{s}^{\prime}, \mathrm{t}^{\prime}\right)
$$

\footnotetext{
${ }^{4}$ In [30], they consider subtraction units of $k \in 2 \mathbb{Z}$ which parametrize both $s$ - and $t$-channel subtractions.
} 


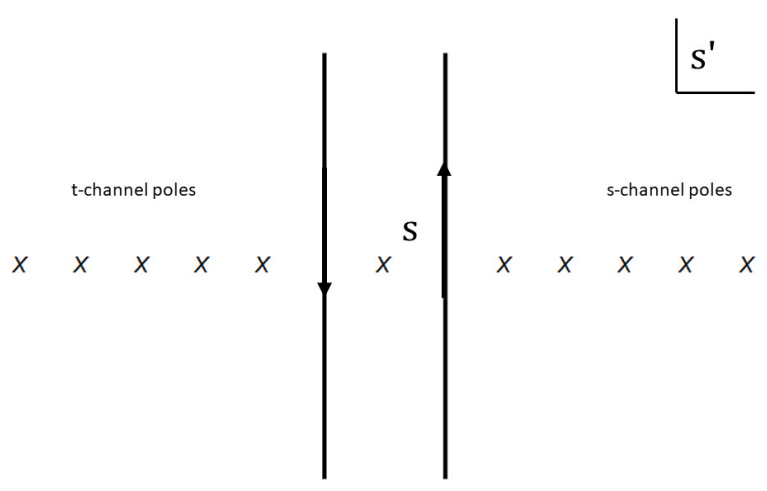

Figure 1. Mellin contours for $M_{\mathbf{k}}^{s}$ and $M_{\mathbf{k}}^{t}$. $s$-channel poles are located at $\mathrm{s}^{\prime}=\tau+2 m$ while $t$-channel poles are located at $\mathrm{s}^{\prime}=s+t-\tau-2 m$ for $m \in \mathbb{Z}$.

where the $\mathrm{s}^{\prime}$-plane is illustrated in figure 1 , and

$$
\begin{aligned}
& M_{\mathbf{k}}^{s}\left(\mathrm{~s}, \mathrm{t} ; \mathrm{s}^{\prime}, \mathrm{t}^{\prime}\right)=\int_{\mathrm{s}+\epsilon-i \infty}^{\mathrm{s}+\epsilon+i \infty} \frac{d \mathrm{~s}^{\prime}}{2 \pi i} \frac{P_{\mathbf{k}}\left(\mathrm{s}, \mathrm{t} ; \mathrm{s}^{\prime}, \mathrm{t}^{\prime}\right) M\left(\mathrm{~s}^{\prime}, \mathrm{t}^{\prime}\right)}{\mathrm{s}-\mathrm{s}^{\prime}} \\
& M_{\mathbf{k}}^{t}\left(\mathrm{~s}, \mathrm{t} ; \mathrm{s}^{\prime}, \mathrm{t}^{\prime}\right)=\int_{\mathrm{s}-\epsilon-i \infty}^{\mathrm{s}-\epsilon+i \infty} \frac{d \mathrm{~s}^{\prime}}{2 \pi i} \frac{P_{\mathbf{k}}\left(\mathrm{s}, \mathrm{t} ; \mathrm{s}^{\prime}, \mathrm{t}^{\prime}\right) M\left(\mathrm{~s}^{\prime}, \mathrm{t}^{\prime}\right)}{\mathrm{s}-\mathrm{s}^{\prime}}=-\int_{\mathrm{t}+\epsilon-i \infty}^{\mathrm{t}+\epsilon+i \infty} \frac{d t^{\prime}}{2 \pi i} \frac{P_{\mathbf{k}}\left(\mathrm{s}, \mathrm{t} ; \mathrm{s}^{\prime}, \mathrm{t}^{\prime}\right) M\left(\mathrm{~s}^{\prime}, \mathrm{t}^{\prime}\right)}{\mathrm{t}-\mathrm{t}^{\prime}} .
\end{aligned}
$$

In the above, we used the identity $\mathrm{s}+\mathrm{t}=\mathrm{s}^{\prime}+\mathrm{t}^{\prime}$ for a fixed-u dispersion relations. To sketch the derivation of the kernel, we restrict ourselves to $\mathbf{k}=0$ for the moment. We seek to express the Mellin amplitude $M\left(\mathrm{~s}^{\prime}, \mathrm{t}^{\prime}\right)$ as the inverse Mellin transform of a positions-space double discontinuity $\operatorname{dDisc}_{s}\left[\mathcal{G}\left(u^{\prime}, v^{\prime}\right)\right]$. The Mellin representation of a correlator $\mathcal{G}(u, v)$ is defined as follows:

$$
\mathcal{G}(u, v)=\iint_{\gamma} \frac{d \mathrm{~s} d \mathrm{t}}{(4 \pi i)^{2}} \Gamma_{\Delta_{i}}^{6}(\mathrm{~s}, \mathrm{t}) u^{\frac{\mathrm{s}-\Delta_{1}-\Delta_{2}}{2}} v^{\frac{\mathrm{t}-\Delta_{2}-\Delta_{3}}{2}} M(\mathrm{~s}, \mathrm{t}),
$$

where $\Gamma_{\Delta_{i}}^{6}(\mathrm{~s}, \mathrm{t})$ is given by eq. (4.3). We wish to substitute the Mellin amplitude defined by eq. (3.14) in the above. Since the double discontinuity multiplies the block by sine factors with zeros at double-twist values, the Mellin amplitude must include corresponding factors that "undo" this feature. We therefore write $M\left(\mathrm{~s}^{\prime}, \mathrm{t}^{\prime}\right)$ evaluated on the $\mathrm{s}^{\prime}$-channel poles as

$$
\begin{aligned}
\left.M\left(\mathrm{~s}^{\prime}, \mathrm{t}^{\prime}\right)\right|_{\mathrm{s}^{\prime}-\text { channel poles }}= & \iint \frac{d u^{\prime} d v^{\prime}}{u^{\prime} v^{\prime}} \frac{u^{\prime \frac{\Delta_{1}+\Delta_{2}-\mathrm{s}^{\prime}}{2}} v^{\prime} \frac{\Delta_{2}+\Delta_{3}-\mathrm{t}^{\prime}}{2}}{2 \sin \left[\frac{\pi}{2}\left(\mathrm{~s}^{\prime}-\Delta_{1}-\Delta_{2}\right)\right] \sin \left[\frac{\pi}{2}\left(\mathrm{~s}^{\prime}-\Delta_{3}-\Delta_{4}\right)\right]} \\
& \times \frac{\mathrm{dDisc}_{s}\left[\mathcal{G}\left(u^{\prime}, v^{\prime}\right)\right]}{\Gamma_{\Delta_{i}}^{6}\left(\mathrm{~s}^{\prime}, \mathrm{t}^{\prime}\right)}
\end{aligned}
$$

After inserting eq. (3.16) into (3.14), we can perform an inverse Mellin transform to return to position-space. By doing so, we arrive at the position-space dispersion relation 
of eq. (1.1) with the following dispersion kernel:

$$
\begin{aligned}
& K_{\mathbf{k}}\left(u, v ; u^{\prime}, v^{\prime}\right)=\frac{1}{u^{\prime} v^{\prime}} \iiint \frac{d \mathrm{~s} d \mathrm{t} d \mathrm{~s}^{\prime}}{(4 \pi i)^{2}(2 \pi i)} \frac{\Gamma_{\Delta_{i}}^{6}(\mathrm{~s}, \mathrm{t}) / \Gamma_{\Delta_{i}}^{6}\left(\mathrm{~s}^{\prime}, \mathrm{t}^{\prime}\right)}{2 \sin \left[\frac{\pi}{2}\left(\mathrm{~s}^{\prime}-\Delta_{1}-\Delta_{2}\right)\right] \sin \left[\frac{\pi}{2}\left(\mathrm{~s}^{\prime}-\Delta_{3}-\Delta_{4}\right)\right]} \\
& \times \frac{u^{\frac{\mathrm{s}-\Delta_{1}-\Delta_{2}}{2}} v^{\frac{\mathrm{t}-\Delta_{2}-\Delta_{3}}{2}}}{u^{\prime \frac{\mathrm{s}^{\prime}-\Delta_{1}-\Delta_{2}}{2}} v^{\frac{\mathrm{t}^{\prime}-\Delta_{2}-\Delta_{3}}{2}}} \frac{P_{\mathbf{k}}\left(\mathrm{s}, \mathrm{t} ; \mathrm{s}^{\prime}, \mathrm{t}^{\prime}\right)}{\mathrm{s}-\mathrm{s}^{\prime}} .
\end{aligned}
$$

The subscript $\mathbf{k}$ emphasizes that this subtracted kernel reconstructs spin-k bounded correlators for any subtraction scheme. The $t$-channel dispersion kernel is obtained by substituting $\frac{d \mathrm{~s}^{\prime}}{\mathrm{s}-\mathrm{s}^{\prime}}$ with $\frac{d \mathrm{t}^{\prime}}{-\mathrm{t}+\mathrm{t}^{\prime}}$ and by replacing the arguments of the sine functions for those of $t$-channel double-twists. From eq. (3.13), it is clear that there is a single kernel for both the $s$ - and $t$-channel correlators, and they must be related to each other by an overall minus sign.

In principle, one can compute $K_{\mathbf{k}}$ to obtain a dispersion relation analogous to that of [29] for spin-k bounded mixed correlators. However, this can be challenging as discussed in appendix A. Our goal is more modest: we aim to compute the collinear functional $B_{\mathbf{k} ; v \mid m n}^{s, t}$ whose kernel is simply eq. (3.17) evaluated on the desired leading $s$-channel pole at $\mathrm{s}=\Delta_{m}+\Delta_{n}+2 k_{m n}$. A closed-form expression for the kernel $\mathfrak{B}_{\mathbf{k} \mid m n}^{\mathfrak{a} \mathfrak{b}}$ is then obtained by deforming the remaining $\mathrm{s}^{\prime}$ and $\mathrm{t}^{\prime}$ contours and re-summing the series; see appendix $\mathrm{A}$ for computational details. By doing so, we find the following expression for the subtracted kernel valid for spin-k bounded mixed correlators:

$$
\begin{aligned}
\mathfrak{B}_{\mathbf{k} \mid 34}^{\mathfrak{a}, \mathfrak{b}}\left(v ; u^{\prime}, v^{\prime}\right)= & \frac{(-1)^{1+k_{12}+k_{34}}}{4 \pi^{3 / 2} u^{\mathfrak{a}+\mathfrak{b}}}\left(\frac{u}{u^{\prime}}\right)^{k_{12}} \frac{v^{\mathfrak{a}+k_{23}} v^{\prime \mathfrak{b}-k_{23}}}{\left(v v^{\prime}\right)^{3 / 2}} \frac{\Gamma(\mathfrak{a}+\mathfrak{b})}{\Gamma\left(\mathfrak{a}+\mathfrak{b}-\frac{1}{2}\right)}\left(u^{\prime}-v-v^{\prime}\right) \chi^{3 / 2-\mathfrak{a}-\mathfrak{b}} \\
& \times{ }_{2} F_{1}\left(\mathfrak{a}, \mathfrak{b},-\frac{1}{2}+\mathfrak{a}+\mathfrak{b},-\frac{1}{\chi}\right)
\end{aligned}
$$

where

$$
\chi=\frac{4 v v^{\prime}}{\left(v-\left(\sqrt{u^{\prime}}+\sqrt{v^{\prime}}\right)^{2}\right)\left(v-\left(\sqrt{u^{\prime}}-\sqrt{v^{\prime}}\right)^{2}\right)},
$$

and

$$
\mathfrak{a}=\frac{1}{2}\left(\Delta_{1}-\Delta_{3}+k_{12}-k_{23}-k_{34}+k_{14}\right), \quad \mathfrak{b}=\frac{1}{2}\left(\Delta_{2}-\Delta_{4}+k_{12}+k_{23}-k_{34}-k_{14}\right) .
$$

The kernel associated with the expansion powers $(m n)=(12)$ can be similarly derived. One can show that the following relation holds:

$$
\mathfrak{B}_{\mathbf{k} \mid 12}^{\mathfrak{a}, \mathfrak{b}}\left(v ; u^{\prime}, v^{\prime}\right)=\left(\frac{u^{\prime}}{u}\right)^{\mathfrak{a}+\mathfrak{b}} \mathfrak{B}_{\mathbf{k} \mid 34}^{\mathfrak{a}, \mathfrak{b}}\left(v ; u^{\prime}, v^{\prime}\right) .
$$

Therefore, the two $s$-channel collinear expansions encode the same information.

A few comments are in order. Firstly, we note that the kernels are antisymmetric under the swapping of $w \leftrightarrow \bar{w}$; this is necessary to be consistent with the sum of $B_{\mathbf{k} ; v \mid m n}^{s}$ and $B_{\mathbf{k} ; v \mid m n}^{t}$ in eq. (2.12). Furthermore, the combination of cross-ratios $\chi$ in eq. (3.18) naturally arises in the $u \rightarrow 0$ limit of the full equal operator dispersion kernel. As shown 
in [29], the equal operator dispersion kernel can be conveniently packaged in terms of the following special combination of cross-ratio variables:

$$
\frac{16 \sqrt{u u^{\prime} v v^{\prime}}}{\left((\sqrt{u}+\sqrt{v})^{2}-\left(\sqrt{u^{\prime}}+\sqrt{v^{\prime}}\right)^{2}\right)\left((\sqrt{u}-\sqrt{v})^{2}-\left(\sqrt{u^{\prime}}-\sqrt{v^{\prime}}\right)^{2}\right)} \underset{u \rightarrow 0}{=} 4 \chi \sqrt{\frac{u u^{\prime}}{v v^{\prime}}}+O(u) .
$$

We expect the special combination of cross-ratios on the l.h.s. to appear in the full mixed correlator Polyakov-Regge block dispersion kernel.

Moreover, we find that the $u$ dependence correctly tracks the corresponding doubletwist trajectories:

$$
\mathfrak{B}_{\mathbf{k} \mid 12}^{\mathfrak{a}, \mathfrak{b}}\left(u, v ; u^{\prime}, v^{\prime}\right) \propto u^{k_{12}}, \quad \mathfrak{B}_{\mathbf{k} \mid 34}^{\mathfrak{a}, \mathfrak{b}}\left(u, v ; u^{\prime}, v^{\prime}\right) \propto u^{\frac{\Delta_{3}+\Delta_{4}-\Delta_{1}-\Delta_{2}+2 k_{34}}{2}} .
$$

We see that subtractions along the $\tau_{12}$ and $\tau_{34}$ trajectories probe subleading double-twists trajectories. We further note that $k_{m n}<0$ is allowed leading to "anti-subtracted" functionals such as the $B_{-2}$ functional introduced in [31] which lead to superconvergent sum rules [45].

Since $\mathfrak{a}, \mathfrak{b} \in \mathbb{R}$, positivity properties are obscured in general. We will revisit this statement in the next subsection after constraining the space of subtraction schemes and operator ordering by verifying convergence conditions. However, we can immediately address the range of allowed $s$-channel subtractions: the latter is determined by the gamma function in the numerator of the kernels. This gamma function arises from the contour deformation in eq. (3.16) of the Mellin-Mandelstam variable s evaluated on the desired $s$-channel double-twist trajectory. It is therefore fixed by the OPE and it is an artefact of taking the $s$-channel collinear limit. For generic subtractions, we have

$$
\mathfrak{B}_{\mathbf{k} \mid 34}^{\mathfrak{a}, \mathfrak{b}} \propto \Gamma\left(\frac{\Delta_{13}+\Delta_{24}}{2}+k_{12}-k_{34}\right), \quad \mathfrak{B}_{\mathbf{k} \mid 12}^{\mathfrak{a}, \mathfrak{b}} \propto \Gamma\left(\frac{-\Delta_{13}-\Delta_{24}}{2}-k_{12}+k_{34}\right) .
$$

The gamma functions diverge for non-positive integer arguments, and therefore the kernels are non-divergent under the following conditions:

$$
\begin{array}{ll}
B_{\mathbf{k} ; v \mid 34}^{s, t}: & k_{12}-k_{34}+\frac{\Delta_{1}+\Delta_{2}-\Delta_{3}-\Delta_{4}}{2} \notin \mathbb{Z}_{\leq 0} \\
B_{\mathbf{k} ; v \mid 12}^{s, t}: & k_{34}-k_{12}+\frac{-\Delta_{1}-\Delta_{2}+\Delta_{3}+\Delta_{4}}{2} \notin \mathbb{Z}_{\leq 0} .
\end{array}
$$

We note that the $\Delta_{i}$ dependence drops out when double-twist operators are exchanged in the $s$-channel. Thus, at least one $s$-channel subtraction is necessary in such cases. In particular, only the $\langle A A B B\rangle$ correlator has unconstrained $s$-channel subtractions.

The conditions above are necessary to obtain non-singular kernels. The divergence caused by the gamma function is a signature of the $\lim _{z \rightarrow 0} \log (z)$ term in the collinear expansion of the correlator. Hence, singular kernels can still be useful sum rules since they define the log coefficient in the $s$-channel collinear expansion. An example of such a functional is the one obtained from the unsubtracted kernel. Log coefficient sum rules are consistent sum rules by themselves, however they may be less practical since the identity is absent in the sum rule. In summary, eq. (3.25) isn't a convergence condition, but rather 
a condition to derive either log or non-log functionals. For the reminder of this paper, we focus on non-log functionals in order to leverage the contribution of the identity.

As an additional consistency check, one can verify that the above expression reduces to the equal operator kernel derived in $[30]^{5}$ in the limit where $\mathfrak{a}=0, \mathfrak{b} \rightarrow k / 2$ for $\mathbf{k}=(k / 2, k / 2,0,0), k \neq 0$ and $(m n)=(34) \cdot{ }^{6} \quad$ Furthermore, we verified the following equivalences:

$$
\mathfrak{B}_{(k / 2, k / 2,0,0) \mid 34}^{0, k / 2}=\mathfrak{B}_{(k / 2,0,0, k / 2) \mid 34}^{k / 2,0}=\mathfrak{B}_{(0, k / 2, k / 2,0) \mid 12}^{-k / 2,0}=\mathfrak{B}_{(0,0, k / 2, k / 2) \mid 12}^{0,-k} .
$$

These equalities foreshadow relations between subtraction schemes.

Lastly, we note that the mixed correlator kernel includes a hypergeometric ${ }_{2} F_{1}$ while the equal operator kernel was a simple function of $u^{\prime}, v^{\prime}$. We explain how the kernel can be simplified for certain pairwise equal operator correlators in appendix B.

\subsection{Convergence and positivity properties: a plethora of subtraction schemes}

In this subsection, we examine the convergence conditions of these functionals associated with different operator ordering and subtraction schemes. It will be helpful to consider the three pairwise equal operator cases separately: $\langle A A B B\rangle,\langle A B B A\rangle,\langle A B A B\rangle$.

The integration region for eq. (3.18) is shown in figure 2. As discussed in [29, 30, 32], the $s$-channel integration region is determined by $\sqrt{v}^{\prime} \geq \sqrt{u}^{\prime}+\sqrt{u}+\sqrt{v}$; this covers a subset of the $L_{u s}$ lightcone provided $\sqrt{u}+\sqrt{v} \geq 1$. Since the $B_{\mathbf{k} ; v \mid m n}$ functional is defined in the $s$-channel collinear limit, we set $u=0$ in the above. If this bound is violated, the integration region includes other spacetime regions and the integral is not guaranteed to be sign definite. The $v^{\prime} \rightarrow \infty$ limit probes the u-channel collinear limit. However, by virtue of the double discontinuity which probes physics on the second sheet, these dispersive transform also captures information from the u-channel Regge limit. The integration boundary therefore includes five dangerous limits: the $s^{-}, t$ - and u-channel collinear limits, the u-channel Regge limit, and the boundary determined by $\sqrt{v}^{\prime} \geq \sqrt{u}^{\prime}+$ $\sqrt{v}$ which we refer to as the lightcone boundary. We summarize all convergence conditions for each of these correlators in table 2 at the end of this subsection.

We first discuss the u-channel Regge limit. We remind the reader that a spin- $J \mathrm{u}-$ channel conformal block scales as $u^{-\Delta_{2}+\Delta 4+J-1}$ in the Regge limit as shown by eq. (3.5). In this limit, the subtracted kernel scales as

$$
\mathfrak{B}_{\mathbf{k} \mid m n}^{\mathfrak{a}, \mathfrak{b}}\left(v ; u^{\prime}, v^{\prime}\right) \underset{\bar{w} \rightarrow+i \infty}{\sim}(\bar{w})^{-1+\Delta_{2}-\Delta_{4}-k_{12}-k_{23}-k_{34}-k_{14}}, \quad w / \bar{w} \text { fixed }
$$

${ }^{5}$ In [30], the $B_{k, v}$ functional is defined as

$$
B_{k, v} \equiv B_{k, v}^{s}+B_{k, v}^{t},
$$

and therefore our results differ by a factor of $1 / 2$. This factor carries over in future results, notably in the derivation of holographic functionals $C_{\mathbf{k} ; \nu \mid 34}^{s, t}$ in eq. (3.57) where the equal operator limit differs by a factor of 2 .

${ }^{6}$ To obtain the unsubtracted kernel associated with the collinear functional $B_{v}^{s}$ in [30], we must take the limit $p \rightarrow 0$ of the sum of both $\mathfrak{B}_{0 \mid 34}^{0, k / 2}$ and $\mathfrak{B}_{0 \mid 12}^{0, k / 2}$ due to the degeneracy of $s$ - and $t$-channel double-twist families in the equal operator limit. 


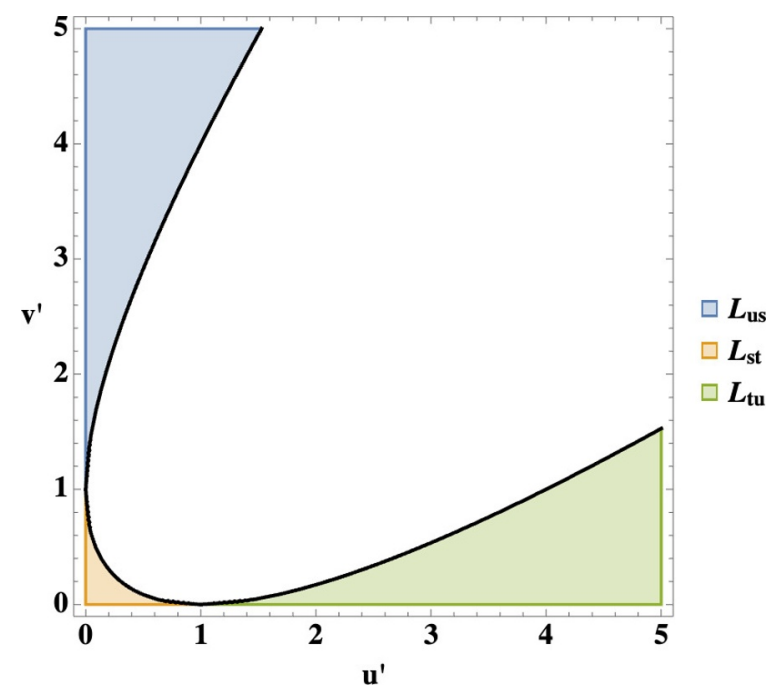

Figure 2. Integration region of dispersive transforms. The black line defined as $\sqrt{v^{\prime}}=\sqrt{u^{\prime}}+\left.\sqrt{v}\right|_{v=1}$ separates the Euclidean region (in white) where $w^{*}=\bar{w}$ and the three lightcones $L_{u s}, L_{s t}$ and $L_{t u}$.
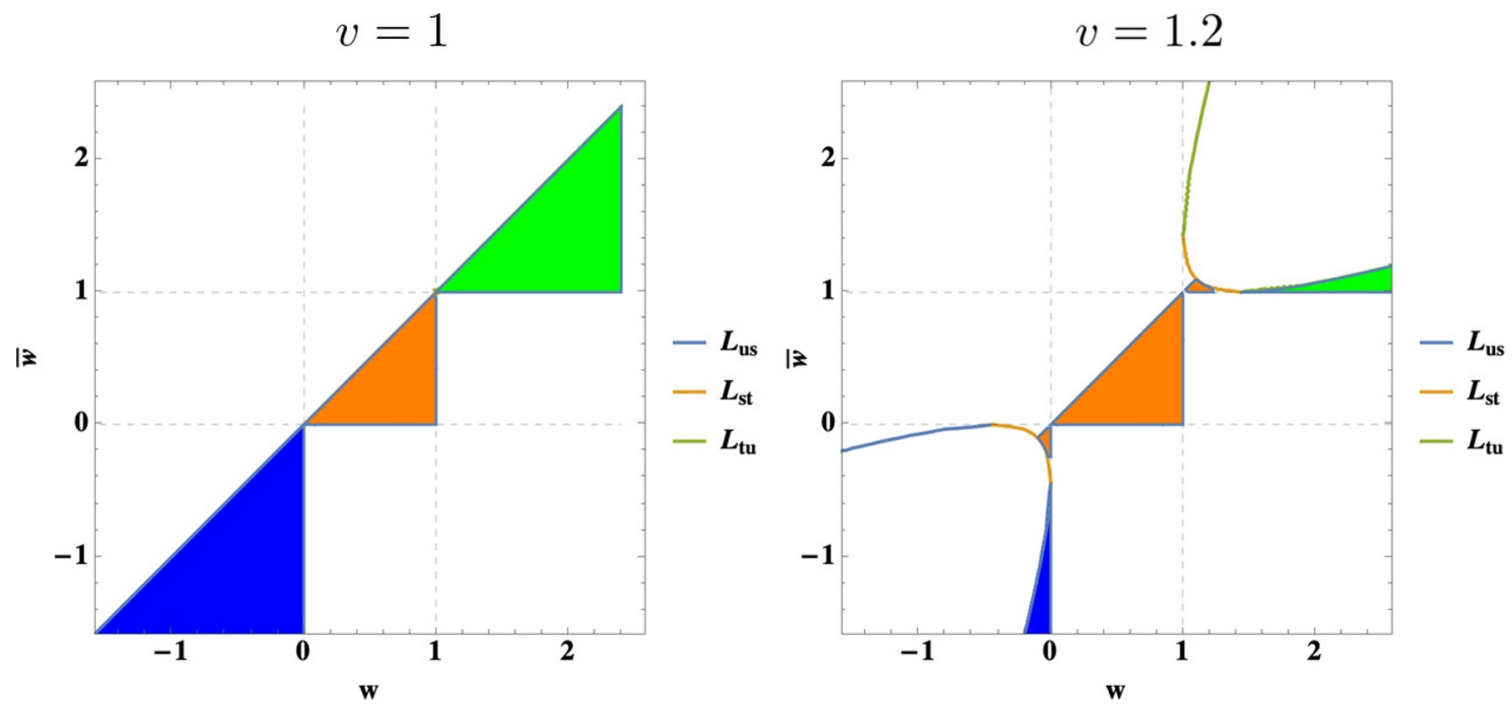

Figure 3. The dispersive transform integration region correspond to the area under the curve where we have illustrated the three Lorentzian regions $L_{u s}=w, \bar{w}<0, L_{s t}=w, \bar{w} \in(0,1)$ and $L_{t u}=w, \bar{w}>1$ for $\bar{w}<w$. The left panel corresponds to $v=1$ while the right panel corresponds to $v=1.2$. The domain of integration lies within the blue $\left(L_{u s}\right)$ and green $\left(L_{t u}\right)$ regions.

and therefore, the Regge boundedness condition is independent of external operator dimensions $\Delta_{i}$.

We discussed the relevance of $s$-channel subtractions in the previous subsection where we found that they serve to differentiate between log and non-log functionals, and to track the $s$-channel collinear expansion. What about $t$-channel subtractions? We will show that the $\mathrm{u}$-channel collinear limit fixes the range of allowed $t$-channel subtractions. 


\begin{tabular}{|c|cc|}
\hline$(m n)$ & $B_{\mathbf{k} ; v \mid m n}^{s}$ & $B_{\mathbf{k} ; v \mid m n}^{t}$ \\
\hline & & \\
$(34)$ & $\tau>\Delta_{1}+\Delta_{2}+2 k_{12}-2$ & $\tau>\min \left(\Delta_{1}+\Delta_{4}+2 k_{14}, \Delta_{2}+\Delta_{3}+2 k_{23}\right)-2$ \\
& & \\
$(12)$ & $\tau>\Delta_{3}+\Delta_{4}+2 k_{34}-2$ & $\tau>\min \left(\Delta_{1}+\Delta_{4}+2 k_{14}, \Delta_{2}+\Delta_{3}+2 k_{23}\right)-2$ \\
\hline
\end{tabular}

Table 1. Collinear limit of the $B_{\mathbf{k} ; v \mid m n}^{s, t}$ functional's integrand. In the equal operator limit, the $s$ and $t$-channel conditions become degenerate.

For our analysis, it is helpful to work in different variables than $u^{\prime}$ and $v^{\prime}$. For convenience, we will map the cross-ratio variables $(w, \bar{w})$ to new variables defined between the interval $(0,1)$ where the hypergeometric functions are naturally defined. If $w, \bar{w}<0$, as shown in figure 3 , we are in the $L_{u s}$ lightcone displayed in blue. Let $(W, \bar{W})=\left(\frac{w}{w-1}, \frac{\bar{w}}{\bar{w}-1}\right)$ such that the $s$-channel dispersive transform becomes

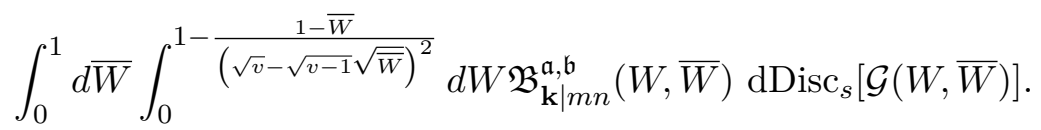

The $t$-channel kinematics are defined for $w, \bar{w}>1$ where the analogous change of variable is $(W, \bar{W})=\left(\frac{w-1}{w}, \frac{\bar{w}-1}{\bar{w}}\right)$. In both cases, the u-channel collinear limit is mapped to $\bar{W}=1$ while the $s$ - and $t$-channel collinear limits are mapped to $W=0$.

Let us evaluate the kernel at the boundaries of integration. In these kinematics, as described in the appendix of [30], the lightcone boundary can be evaluated by analytic continuation from a regime where the integrand is convergent. Therefore, convergence is unaffected by the lightcone boundary limit.

We record for convenience the behaviour of the $s$-channel collinear limit $W \rightarrow 0$ of $B_{\mathbf{k} ; v \mid m n}^{s}$ where the domain is in the lightcone $L_{u}$ :

$$
L_{u s}: \quad \mathfrak{B}_{\mathbf{k} \mid 34}^{\mathfrak{a}, \mathfrak{b}}(W, \bar{W}) \underset{W \rightarrow 0}{\sim}(W \bar{W})^{-k_{12}}, \quad \mathfrak{B}_{\mathbf{k} \mid 12}^{\mathfrak{a}, \mathfrak{b}}(W, \bar{W}) \underset{W \rightarrow 0}{\sim}(W \bar{W})^{\frac{\Delta_{1}+\Delta_{2}-\Delta_{3}-\Delta_{4}}{2}-k_{34}} .
$$

Due to the antisymmetry in $W \leftrightarrow \bar{W}$, the kernel behaves similarly in the $t$-channel collinear limit $\bar{W} \rightarrow 0$ limit. To probe the $t$-channel collinear limit of $B_{\mathbf{k} ; v \mid 34}^{t}$, we note that the change of variable for $W, \bar{W}$ is different in the lightcone $L_{t u}$ such that both kernels behave as follows:

$$
L_{t u}: \quad \mathfrak{B}_{\mathbf{k} \mid 34}^{\mathfrak{a}, \mathfrak{b}}(W, \bar{W}), \mathfrak{B}_{\mathbf{k} \mid 12}^{\mathfrak{a}, \mathfrak{b}}(W, \bar{W}) \underset{W \rightarrow 0}{\sim}(W \bar{W})^{-k_{23}}+(W \bar{W})^{\frac{\Delta_{21}+\Delta_{34}}{2}-k_{14}} .
$$

We note that for equal operators, the subtraction units $k_{i j}$ become degenerate. After accounting for the contribution of the conformal blocks from eq. (3.3), we conclude that the dispersive transform is guaranteed to be convergent and sign definite according to table 1 . Below these thresholds, the functionals are defined by analytic continuation and therefore, they are no longer guaranteed to be sign definite. Implementing the analytic continuation is subtle. The reader is encouraged to consult appendix D of [30] to learn more.

Since the kernel is antisymmetric in $W \leftrightarrow \bar{W}$, we describe the final limit of interest: the u-channel collinear limit $\bar{W} \rightarrow 1$. To determine the convergence conditions, we probe the 


\begin{tabular}{|c|c|c|}
\hline Functional & $s$-channel subtractions & $t$-channel subtractions \\
\hline & & $2 k_{14}>\Delta_{2}-\Delta_{4}-2 k_{34}$ \\
$B_{\mathbf{k} ; v \mid 34}^{s, t}$ & $k_{12}-k_{34}+\Delta_{1}+\Delta_{2}-\Delta_{3}-\Delta_{4} \notin \mathbb{Z}_{\leq 0}$ & $2 k_{23}>\Delta_{1}-\Delta_{3}-2 k_{34}$ \\
& & $2 k_{14}>-\Delta_{2}+\Delta_{4}-2 k_{12}$ \\
$B_{\mathbf{k} ; v \mid 12}^{s, t}$ & $k_{34}-k_{12}-\Delta_{1}-\Delta_{2}+\Delta_{3}+\Delta_{4} \notin \mathbb{Z}_{\leq 0}$ & $2 k_{23}>-\Delta_{1}+\Delta_{3}-2 k_{12}$ \\
\hline
\end{tabular}

Table 2. Convergence conditions for non-log functionals determined by subtraction units $k_{i j} \in$ $\mathbb{Z}$. $s$-channel subtractions determine whether the functionals are log or non-log, while $t$-channel subtractions determine convergence conditions. The $t$-channel condition becomes eq. (3.36) for the $\langle A B A B\rangle$ correlator if we consider the $\mathcal{G}-1$ correlator. Lastly, we note that "anti-subtractions" are possible for $k_{i j}<0$.

u-channel collinear limit by taking $x_{2} \rightarrow x_{4}$ and $x_{1} \rightarrow x_{3}$. The behaviour of the correlator can be easily assessed by considering eq. (3.1): by working in radial coordinates $\rho$ where $\rho \rightarrow 1$ is the $\mathrm{u}$-channel collinear limit, the stripped factor is polynomially singular ${ }^{7}$

$$
\left\langle\phi_{1} \phi_{2} \phi_{3} \phi_{4}\right\rangle \sim(1-\rho)^{-\frac{\Delta_{1}+3 \Delta_{2}+\Delta_{3}-\Delta_{4}}{2}} \mathcal{G}(\rho, \bar{\rho}) .
$$

The stripped correlator can then be bounded using Cauchy-Schwarz [3, 4]. We therefore conclude that

$$
|\mathcal{G}(\rho, \bar{\rho})| \leq C(1-\rho)^{\Delta_{2}-\Delta_{4}}
$$

for some $C>0$.

In this limit, the kernels behave as

$$
\begin{aligned}
& \frac{\mathfrak{B}_{\mathbf{k} \mid 34}^{\mathfrak{a}, \mathfrak{b}}(\rho, \bar{\rho})}{u^{-\frac{\Delta_{2}-\Delta_{4}}{2}}} \underset{\rho \rightarrow 1}{\sim}(1-\rho)^{-\Delta_{1}-3 \Delta_{2}+2 \Delta_{4}+2 k_{34}-1}\left((1-\rho)^{\Delta_{2}+\Delta_{3}+2 k_{23}}+(1-\rho)^{\Delta_{1}+\Delta_{4}+2 k_{14}}\right), \\
& \frac{\mathfrak{B}_{\mathbf{k} \mid 12}^{\mathfrak{a}, \mathfrak{b}}(\rho, \bar{\rho})}{u^{-\frac{\Delta_{2}-\Delta_{4}}{2}}} \underset{\rho \rightarrow 1}{\sim}(1-\rho)^{-2 \Delta_{2}-\Delta_{3}+2 k_{34}-1}\left((1-\rho)^{\Delta_{2}+\Delta_{3}+2 k_{23}}+(1-\rho)^{\Delta_{1}+\Delta_{4}+2 k_{14}}\right), \quad(3.35)
\end{aligned}
$$

where we have divided by the u-channel identity to make manifest the convergence conditions. We note that the symmetry between the $k_{23}$ and $k_{14}$ subtractions are due to hypergeometric function in eq. (3.18). Together, the last two equations determine convergence conditions in the $\mathrm{u}$-channel collinear limit.

By combining all of the previous discussions, we can derive the convergence conditions summarized in the following table for non-log functionals. We note that the $t$-channel condition can be relaxed for the $\langle A B A B\rangle$ correlator if we consider the $\mathcal{G}-1$ correlator. In this case where the $t$-channel convergence conditions are marginally satisfied for $k_{i j}=0$, we

\footnotetext{
${ }^{7}$ Explicitly, we work in a configuration where all the points $x_{i}$ lie in a 2-plane with complex coordinates $y=x^{1}+i x^{2}, \bar{y}=x^{1}-i x^{2}$, and the operators are positioned at

$$
\left\langle\phi_{1}(-\rho,-\bar{\rho}) \phi_{2}(\rho, \bar{\rho}) \phi_{3}(-1,-1) \phi_{4}(1,1)\right\rangle,
$$

such that $\rho \rightarrow 1$ corresponds to the u-channel collinear limit.
} 
assume that the subleading $\mathrm{u}$-channel collinear singularity comes from a $\mathrm{u}$-channel block such that the functionals $B_{\mathbf{k} ; v \mid m n}^{s, t}$ converge provided that

$$
2 k_{14}>-\min \left(\Delta_{A}, \Delta_{B}\right)-2 k_{m n}, \quad 2 k_{23}>-\min \left(\Delta_{A}, \Delta_{B}\right)-2 k_{m n} .
$$

In other words, $t$-channel subtractions are unnecessary for the $\langle A B A B\rangle$ correlator unless $k_{m n}<0$. Furthermore, the convergence conditions in table 2 illustrates how different operator ordering and subtraction schemes may be related. For example, applying a dispersive transform with the kernel $\mathfrak{B}_{\left(r_{1}, k_{23}, r_{2}, k_{14}\right) \mid 34}$ to the $\langle A A B B\rangle$ correlator is equivalent to using $\mathfrak{B}_{\left(r_{2}, k_{23}, r_{1}, k_{14}\right) \mid 12}$ kernel for the $\langle B B A A\rangle$ correlator; this follows from eq. (3.21). We will test the convergence conditions in table 2 by exploring different subtraction schemes in section 5.1 for mixed correlators of the 3D Ising model.

Let us consider an example to illustrate these convergence conditions. We consider the $B_{\mathbf{k} ; v \mid 34}^{s}$ functional acting on the $\langle A B B A\rangle$ correlator with external operators $\sigma$ and $\epsilon$ from the 3D Ising model. The collinear limit of the integrand behaves as follows:

$$
\begin{aligned}
& \mathfrak{B}_{\mathbf{k} \mid 34}\langle\sigma \epsilon \epsilon \sigma\rangle \sim(1-\rho)^{-1.9+2 k_{14}+2 k_{34}}+(1-\rho)^{-0.1+k_{23}+2 k_{34}} \\
& \mathfrak{B}_{\mathbf{k} \mid 34}\langle\epsilon \sigma \sigma \epsilon\rangle \sim(1-\rho)^{-0.1+2 k_{14}+2 k_{34}}+(1-\rho)^{-1.9+k_{23}+2 k_{34}}
\end{aligned}
$$

A single $t$-channel subtraction along the $\tau_{14}$ double-twist trajectory is necessary for the $\langle\sigma \epsilon \epsilon \sigma\rangle$ while a subtraction is necessary along the $\tau_{23}$ trajectory for the $\langle\epsilon \sigma \sigma \epsilon\rangle$. This example highlights relations between operator ordering and subtraction schemes.

We further underscore the possibility for "anti-subtractions" according to table 2, i.e. $k_{i j}<0$. In particular, for equal operators, the $\mathbf{k}=(0,-1,-1,0)$ subtraction scheme, or equivalently $(0,0,-1,-1)$, applied to the $B_{\mathbf{k} ; v \mid 34}$ functional corresponds to the antisubtracted functional $B_{-2}$ introduced in [31]. We will show that anti-subtractions change the Mellin-space domain of convergence.

Finally, we revisit the positivity properties of our dispersive transforms. First, we note that the hypergeometric function in eq. (3.18) is sign definite in the integration range, and therefore the integral is guaranteed to be positive according to the inequalities shown in table 1. As previously discussed, the double discontinuity defines a positive definite matrix in terms of the pairs of external operators 12 and 34. We therefore consider each pairwise equal operator correlator separately.

For the $\langle A B A B\rangle$ correlator, the double discontinuity returns a $\sin ^{2}$ and hence it is positive definite in both channels. Given the convergence conditions, we further conclude that the dispersive transform is also positive. Thus, any negativity stemming from this such sum rules must be due to an operator below the first double-twist value $\Delta_{A}+\Delta_{B}+\ell$, or they are due to the OPE coefficient which is proportional to $f_{B A \mathcal{O}}=f_{A B \mathcal{O}}(-1)^{\ell_{\mathcal{O}}}$. Thus, even and spin trajectories are individually sign definite, but their sum is not. Moreover, as opposed to the other two cases, the sum rule adds up to the u-channel identity rather than vanishing.

For the $\langle A B B A\rangle$ correlator, the double discontinuity also returns a $\sin ^{2}$ in the $s$ channel, but they are the product of two independent sin functions in the $t$-channel. Thus, the $s$-channel sector of the sum rule is positive for $\tau>\Delta_{A}+\Delta_{B}+2 k_{i j}-2$ for a number of $s$-channel subtractions $k_{i j}$; it is no longer sign definite in the $t$-channel. The $\langle A A B B\rangle$ 


\begin{tabular}{|ccc|}
\hline & $\mathrm{AdS}_{d+1}$ & $\mathrm{CFT}_{d}$ \\
\hline angular momentum & $J$ & $J$ \\
energy & $m$ & $(\Delta-J-d+1)(\Delta+J-1)$ \\
impact paramter & $\beta=\cosh ^{-1}\left(\eta_{\text {AdS }}\right)$ & $\log \frac{\Delta+J-1}{\Delta-J+d-1}$ \\
Regge limit & $m \gg 1$ at fixed $\beta$ & $\Delta \gg 1$ at fixed $J / \Delta$ \\
bulk-point limit & $m \gg 1$ at fixed $J$ & $\Delta \gg 1$ at fixed $J$ \\
\hline
\end{tabular}

Table 3. Dictionary between AdS and CFT quantum numbers.

correlator behaves similarly but with $s$ - and $t$-channel positivity properties exchanged. A key different between the $\langle A A B B\rangle$ and $\langle A B B A\rangle$ correlators is the allowed range of $s$ channel subtractions to produce non-log functionals. These properties will be verified in section 5.1 where we evaluate sum rules of various correlators of the 3D Ising model.

To summarize this section, $s$-channel subtractions track the $s$-channel collinear expansion and therefore distinguish between log and non-log functionals. On the other hand, $t$-channel subtractions are necessary to control the behaviour of the correlator in the uchannel collinear limit. These conditions are summarized in table 2. Positivity on the other hand is controlled by $s$ - and $t$-channel double-twist operators and their corresponding subtractions. Given our newfound understanding of these subtraction schemes and convergence properties, we will restrict ourselves to the $B_{\mathbf{k} ; v \mid 34}$ functional for the rest of this paper.

\subsection{Mixed correlator holographic functionals $C_{\mathrm{k} ; \nu \mid m n}$}

In [31], the authors introduced a dispersive functional $C_{k, \nu}$ which localizes physics in AdS spacetime motivated by previous work conducted in flat-space [37]. The goal was to relate AdS and CFT quantum numbers to probe local physics in bulk AdS. This is achieved by localizing wavepackets at fixed impact parameter in the bulk-point limit. The idea is to parametrize $2 \rightarrow 2$ scattering by labelling states with conserved quantities such as the impact parameter $b=2 \mathrm{~J} / \mathrm{m}$ conjugate to the transverse momentum $u=-p^{2}$. In AdS, pairs of particles with large center-of-mass energy $\Delta / R_{\text {AdS }}$ separated by a geodesic distance of $\beta R_{\text {AdS }}$ carry total angular momentum

$$
J=\Delta \tanh \left(\frac{\beta}{2}\right) .
$$

We therefore see that local physics, described by the bulk point limit where the r.h.s. vanishes, is obtained by taking $J / \Delta \ll 1$ for $\Delta \gg 1$. The Regge limit is reached by taking $\Delta$ large for fixed $J / \Delta$. We review other parameters of this dictionary in table 3.

These so-called holographic functionals $C_{k, \nu}$ exhibit the following properties:

- In the Regge limit where $\Delta$ is the largest parameter, they are proportional to AdS

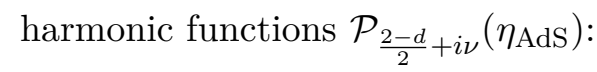

$$
C_{k, \nu}[\Delta, J] \propto \frac{\mathcal{P}_{\frac{2-d}{2}+i \nu}\left(\frac{\Delta^{2}+J^{2}}{\Delta^{2}-J^{2}}\right)}{m^{2 k}} \times\left(1+O\left(\frac{\nu^{2}}{m^{2}}, \frac{1}{m^{2}}\right)\right) .
$$


- In the bulk-point limit where $\nu \in[0, m)$, they encode the contribution of massive states in a partial wave expansion:

$$
C_{k, \nu}[\Delta, J] \propto \mathcal{P}_{J}\left(1-\frac{2 \nu^{2}}{m^{2}}\right) \times\left(1+O\left(\frac{J^{2}}{m^{2}}\right)\right),
$$

where $u R_{\text {AdS }}^{2}=-\nu^{2}$.

- The action on light operators matches that of the flat-space dispersion relation in the large $\nu$ (small impact parameter) limit

$$
-\left.C_{k, \nu}\right|_{\text {light }}=-\mathcal{C}_{k, u} \times\left(1+O\left(\frac{1}{\nu^{2}}\right)\right) .
$$

Such functionals lead to holographic sum rules providing an additional pathway to bootstrap quantum gravity in AdS from CFTs. While such a bootstrap exercise extends beyond the goal of this paper, given our goal to derive mixed correlator dispersive CFT functionals, we will generalize the previous construction to mixed correlators.

We first sketch the derivation of these holographic functionals $C_{k, \nu}$ based on the work of $[31]$ :

1. Given the physical functional $B_{k, v}$, we write position-space dispersive transform in terms of the radial and angular coordinates $r$ and $\eta$

$$
B_{k, v}=\iint d r d \eta \mathfrak{B}_{k}(v ; r, \eta) \mathrm{dDisc}\left[G_{\Delta, J}(r, \eta)\right] \propto \int \frac{\eta d \eta}{\left(\eta^{2}-v\right)^{\frac{3-k}{2}}} \Pi_{k, \eta}+O\left(\Pi_{k+2, \eta}\right),
$$

where we have expanded the integrand in terms of Regge moments

$$
\Pi_{k, \eta}[\mathcal{G}]=\int_{0}^{r_{\max }(\eta)} d r r^{k-2} \operatorname{dDisc}_{s} \mathcal{G}(r, \eta)
$$

2. We can extract a Regge moment functional by performing an inverse transform on $B_{k, v}$ :

$$
\Pi_{k, \eta}\left[G_{\Delta, J}\right] \propto \int_{\eta^{2}}^{\infty} \frac{d v}{\left[\left(v-\eta^{2}\right)^{\frac{k+1}{2}}\right]_{+}} B_{k, v},
$$

where the + subscript denotes a distribution where we have subtracted singular terms around $v=\eta^{2}$. Unfortunately, this functional is delocalized in impact parameter and therefore fails to describe bulk AdS physics.

3. To solve this, one can localize in transverse impact parameter $\eta$ by performing harmonic analysis on the transverse space $H_{d-1}$ in AdS:

$$
C_{k, \nu} \propto \int_{1}^{\infty}[d \eta] \mathcal{P}_{\frac{2-d}{2}+i \nu}(\eta) \int_{\eta^{2}}^{\infty} \frac{d v}{\left[\left(v-\eta^{2}\right)^{\frac{k+1}{2}}\right]_{+}} B_{k, v}
$$

where the proportionality factor is fixed by matching the functional with the flatspace dispersion relation $\mathcal{C}_{k, u}$. 
We will now extend this position-space derivation to mixed correlators; a Mellin-space derivation will be detailed in section 4.2 .

Let us define the radial coordinates from [46] adapted in the u-channel as follows:

$$
\rho=\frac{1}{(\sqrt{1-w}+\sqrt{-w})^{2}}, \quad \bar{\rho}=\frac{1}{(\sqrt{1-\bar{w}}+\sqrt{-\bar{w}})^{2}},
$$

We define the radial and angular coordinates as

$$
r \equiv \sqrt{\rho \bar{\rho}}, \quad \eta \equiv \frac{\rho+\bar{\rho}}{2 \sqrt{\rho \bar{\rho}}} .
$$

In these new coordinates, the dispersive transform reads

$$
\begin{aligned}
B_{\mathbf{k} ; v \mid 34}^{s, t} & =\int_{\sqrt{v}}^{\infty} d \eta \int_{0}^{r_{\max }(\eta)} d r \mathfrak{B}_{\mathbf{k} \mid 34}^{\mathfrak{a}, \mathfrak{b}}(v ; \eta, r) \operatorname{dDisc}_{s, t}[\mathcal{G}] \\
& =\int_{\sqrt{v}}^{\infty} d \eta\left(H_{0}(v, \eta) \Pi_{\mathbf{k}, 0, \eta}^{s, t}[\mathcal{G}]+H_{1}(v, \eta) \Pi_{\mathbf{k}, 1, \eta}^{s, t}[\mathcal{G}]+O\left(\Pi_{\mathbf{k}, 2, \eta}^{s, t}[\mathcal{G}]\right)\right)
\end{aligned}
$$

where $r_{\max }=\eta-\sqrt{\eta^{2}-1}$. Similar to the equal operator case, we define Regge moments of mixed correlators $\Pi_{\mathbf{k}, n, \eta}^{s, t}$ as the following expansion of the kernel in the $\mathrm{u}$-channel Regge limit $r \rightarrow 0$ :

$$
\Pi_{\mathbf{k}, n, \eta}^{s, t}[\mathcal{G}] \equiv \int_{0}^{r_{\max }(\eta)} d r r^{-\Delta_{2}+\Delta_{4}+k_{12}+k_{23}+k_{34}+k_{14}+n-2} \operatorname{dDisc}_{s, t}[\mathcal{G}] .
$$

We use a slightly different notation than in [31] since different subtraction schemes will play a role in our analysis; $n$ labels the moment relative to the total spin convergence.

The next step is to invert eq. (3.48) to extract the Regge moment functionals. To achieve this, we explicitly write the expansion of $B_{\mathbf{k} ; v \mid 34}$ up to leading order in the Regge moment:

$$
\begin{aligned}
B_{\mathbf{k} ; v \mid 34}^{s, t}= & \int_{\sqrt{v}}^{\infty} d \eta \frac{(-1)^{1+k_{12}+k_{34}}}{2 \pi^{3 / 2} v^{k_{12}}} \frac{\eta(4 \sqrt{v})^{-\Delta_{2}+\Delta_{4}+k_{12}+k_{14}+k_{23}+k_{34}}}{\left(\eta^{2}-v\right)^{\frac{3}{2}-\mathfrak{a}-\mathfrak{b}}} \frac{\Gamma(\mathfrak{a}+\mathfrak{b})}{\Gamma\left(-\frac{1}{2}+\mathfrak{a}+\mathfrak{b}\right)} \\
& \times{ }_{2} F_{1}\left(\mathfrak{a}, \mathfrak{b},-\frac{1}{2}+\mathfrak{a}+\mathfrak{b}, 1-\frac{\eta^{2}}{v}\right) \Pi_{\mathbf{k}, 0, \eta}^{s, t}+O\left(\Pi_{\mathbf{k}, 1, \eta}^{s, t}\right),
\end{aligned}
$$

where $\mathfrak{a}, \mathfrak{b}$ is given by eq. (3.20). We note that the $\eta$ dependence in the first line is analogous to the equal operator case where the authors in [31] found $\frac{\eta}{\left(\eta^{2}-v\right)^{(3-k) / 2}}$. Furthermore, the equal operator limit is given by $(\mathfrak{a}, \mathfrak{b}) \rightarrow(0, k / 2)$ or $(k / 2,0)$. The major distinction between the two cases is the presence of the hypergeometric function in the second line above. Fortunately, the $\eta$ integral can be done analytically ${ }^{8}$ and the result is simply a polynomial

\footnotetext{
${ }^{8}$ The integral can be reduced to the following form under an appropriate change of variables:
}

$$
\int_{0}^{\infty} d x(1+x)^{d} x_{2}^{c} F_{1}(a, b, c+1,-x) \text {. }
$$


in $v$ with additional gamma functions. Therefore, like the equal operator case, an inverse transform exists where for some power $p$ of $\eta^{p}$, we have

$$
\begin{aligned}
\int_{\sqrt{v}}^{\infty} d \eta & \frac{\eta^{1+p}}{\left(\eta^{2}-v\right)^{\frac{3}{2}-\mathfrak{a}-\mathfrak{b}}}{ }^{2} F_{1}\left(\mathfrak{a}, \mathfrak{b},-\frac{1}{2}+\mathfrak{a}+\mathfrak{b}, 1-\frac{\eta^{2}}{v}\right) \\
\quad= & \frac{v^{\frac{1}{2}(-1+p)+\mathfrak{a}+\mathfrak{b}}}{2} \frac{\Gamma\left(-\frac{1}{2}+\mathfrak{a}+\mathfrak{b}\right) \Gamma\left(\frac{1}{2}-\mathfrak{a}-\frac{p}{2}\right) \Gamma\left(\frac{1}{2}-\mathfrak{b}-\frac{p}{2}\right)}{\Gamma\left(\frac{1-p}{2}\right) \Gamma\left(-\frac{p}{2}\right)}
\end{aligned}
$$

and an inverse exists of the form

$$
\begin{aligned}
& \int_{\eta^{2}}^{\infty} d v \frac{v^{\frac{1}{2}(-1+p)+\mathfrak{a}+\mathfrak{b}}}{\left(v-\eta^{2}\right)^{\frac{1}{2}+\mathfrak{a}+\mathfrak{b}}}\left(\frac{v}{\eta^{2}}\right)^{-\mathfrak{b}}{ }_{2} F_{1}\left(\frac{1}{2}-\mathfrak{b},-\mathfrak{b}, \frac{1}{2}-\mathfrak{a}-\mathfrak{b}, 1-\frac{v}{\eta^{2}}\right) \\
& \quad=\eta^{p} \frac{\Gamma\left(\frac{1}{2}-\mathfrak{a}-\mathfrak{b}\right) \Gamma\left(\frac{1}{2}-\frac{p}{2}\right) \Gamma\left(-\frac{p}{2}\right)}{\Gamma\left(\frac{1-p}{2}-\mathfrak{a}\right) \Gamma\left(\frac{1-p}{2}-\mathfrak{b}\right)} .
\end{aligned}
$$

By combining the two relations above, we obtain an inverse transform for the leading Regge moment:

$$
\int_{\eta^{2}}^{\infty} d v \frac{2 v^{\frac{1}{2}\left(\Delta_{2}-\Delta_{4}+k_{12}-k_{14}-k_{23}-k_{34}\right)}\left(v / \eta^{2}\right)^{-\mathfrak{b}}}{\left(v-\eta^{2}\right)^{\frac{1}{2}+\mathfrak{a}+\mathfrak{b}}} \frac{F_{1}\left(\frac{1}{2}-\mathfrak{b},-\mathfrak{b}, \frac{1}{2}-\mathfrak{a}-\mathfrak{b}, 1-\frac{v}{\eta^{2}}\right)}{\Gamma\left(\frac{1}{2}-\mathfrak{a}-\mathfrak{b}\right) \Gamma\left(-\frac{1}{2}+\mathfrak{a}+\mathfrak{b}\right)} B_{\mathbf{k} ; v \mid 34}^{s, t} \sim \Pi_{\mathbf{k}, 0, \eta}^{s, t}
$$

The final step in our derivation consists of localizing in impact parameter which is achieved by performing harmonic analysis in the transverse space $H_{d-1}$. We proceed as in the equal operator case by first projecting the Regge moment functional against $\mathrm{SO}(d-1,1)$ partial waves

$$
\widehat{\Pi}_{\mathbf{k} ; 0, \nu \mid 34}^{s, t}=\int_{1}^{\infty}[d \eta] \mathcal{P}_{\frac{2-d}{2}+i \nu}(\eta) \Pi_{\mathbf{k}, 0 ; \eta \mid 34}^{s, t}, \quad \text { where }[d \eta] \equiv 2^{d-2}\left(\eta^{2}-1\right)^{\frac{d-3}{2}} d \eta,
$$

where

$$
\mathcal{P}_{J}={ }_{2} F_{1}\left(-J, J+d-2, \frac{d-1}{2}, \frac{1-x}{2}\right) .
$$

Thus, our holographic functional reads

$$
\begin{aligned}
C_{\mathbf{k} ; \nu \mid 34}^{s, t}= & (-1)^{k_{12}+k_{34}+1} 4^{1+\Delta_{2}-\Delta_{4}-k_{12}-k_{23}-k_{34}-k_{14}} \pi^{3 / 2} a_{\Delta_{i}, k_{i j}}(\nu) \int_{1}^{\infty}[d \eta] \mathcal{P}_{\frac{2-d}{2}+i \nu}(\eta) \\
& \times \int_{\eta^{2}}^{\infty} d v \frac{\left(v / \eta^{2}\right)^{-\mathfrak{b}} v^{\frac{1}{2}\left(\Delta_{2}-\Delta_{4}+k_{12}-k_{14}-k_{23}-k_{34}\right)}}{\left(v-\eta^{2}\right)^{\frac{1}{2}+\mathfrak{a}+\mathfrak{b}}} \frac{{ }_{2} F_{1}\left(\frac{1}{2}-\mathfrak{b},-\mathfrak{b}, \frac{1}{2}-\mathfrak{a}-\mathfrak{b}, 1-\frac{v}{\eta^{2}}\right)}{\Gamma\left(\frac{1}{2}-\mathfrak{a}-\mathfrak{b}\right) \Gamma(\mathfrak{a}+\mathfrak{b})} B_{\mathbf{k} ; v \mid 34}^{s, t}
\end{aligned}
$$

where the normalization factor $a_{\Delta_{i}, k_{i j}}(\nu)$ is defined as

$$
a_{\Delta_{i}, k_{i j}}(\nu)=\frac{\pi^{\frac{d-3}{2}}\left(\prod_{i=1}^{4} \sqrt{\Gamma\left(\Delta_{i}\right) \Gamma\left(\Delta_{i}+1-d / 2\right)}\right) /\left(2^{d-4} \Gamma\left(\frac{d-1}{2}\right)\right)}{\gamma_{\Delta_{1}+\Delta_{3}+k_{12}+k_{14}+k_{23}-k_{34}-1}(\nu) \gamma_{\Delta_{2}+\Delta_{4}+k_{12}+k_{14}+k_{23}-k_{34}-1}(\nu)},
$$


and

$$
\gamma_{a}(\nu) \equiv \Gamma\left(\frac{1+a-d / 2-i \nu}{2}\right) \Gamma\left(\frac{1+a-d / 2+i \nu}{2}\right) .
$$

This normalization factor is fixed by relating the Mellin amplitude to the flat-space scattering amplitude based on the convention in [13]:

$$
M(\mathrm{~s}, \mathrm{t})=\frac{1}{8 \pi^{d / 2} \prod_{i=1}^{4} \sqrt{\Gamma\left(\Delta_{i}\right) \Gamma\left(\Delta_{i}+1-d / 2\right)}} \int_{0}^{\infty} d \beta \beta^{\frac{\sum_{i} \Delta_{i}}{2}-d / 2-1} e^{-\beta} \mathcal{M}_{\mathrm{flat}}(2 \beta \mathrm{s}, 2 \beta \mathrm{t}),
$$

and the $\gamma_{a}(\nu)$ dependence will be justified by our derivation of the Mellin-space functional in section 4.2 where we will further elaborate on the $\Delta_{i}$ dependence. One can verify that eq. (3.57) matches the result of [31] in the equal operator limit.

A similar $\gamma_{\Delta_{i}}(\nu)$ factor was also present in the equal operator case. We expect this factor to be the only $\nu$-dependent term in the Regge moment $\widehat{\Pi}_{\mathbf{k} ; 0, v \mid 34}^{s, t}$ :

$$
\widehat{\Pi}_{\mathbf{k} ; 0, v \mid 34}^{s, t} \propto \gamma_{\Delta_{1}+\Delta_{3}+k_{12}+k_{14}+k_{23}-k_{34}-1}(\nu) \gamma_{\Delta_{2}+\Delta_{4}+k_{12}+k_{14}+k_{23}-k_{34}-1}(\nu) \mathcal{P}_{\frac{2-d}{2}+i \nu}(\eta) .
$$

Physically, these gamma functions are necessary to "undo" the smearing of these dispersive transforms in order to localize the scattering processes in the bulk. This insight is made manifest in Mellin-space. Moreover, because these dispersion relations are derived in a fixed-u convention, the $\Delta_{i}$ should reflect the exchange of u-channel double-twists.

What about higher order Regge moments? By expanding the $\mathfrak{B}_{\mathbf{k} \mid 34}^{\mathfrak{a}, \mathfrak{b}}$ kernel to subleading order in $r$, we find

$$
\begin{aligned}
\left.B_{\mathbf{k} ; v \mid 34}^{s, t}\right|_{r^{1}}= & \int_{\sqrt{v}}^{\infty} d \eta \frac{(-1)^{1+k_{12}+k_{34}}}{2 \pi^{3 / 2} v^{k_{12}}} \frac{\eta(4 \sqrt{v})^{-\Delta_{2}+\Delta_{4}+k_{12}+k_{14}+k_{23}+k_{34}}}{\left(\eta^{2}-v\right)^{\frac{1}{2}(-3+\mathfrak{a}+\mathfrak{b})} \frac{\Gamma(\mathfrak{a}+\mathfrak{b})}{\Gamma\left(-\frac{1}{2}+\mathfrak{a}+\mathfrak{b}\right)}} \\
& \times\left(-\frac{8 \mathfrak{a} \mathfrak{b}\left(\eta^{2}-v\right) \eta^{2}}{v(-1+2 \mathfrak{a}+2 \mathfrak{b})}{ }_{2} F_{1}\left(1+\mathfrak{a}, 1+\mathfrak{b}, \frac{1}{2}+\mathfrak{a}+\mathfrak{b}, 1-\frac{\eta^{2}}{v}\right)\right. \\
& \left.+\left(-2 v+2 \eta^{2}\left(2 \mathfrak{a}-2 k_{12}+k_{23}\right)\right){ }_{2} F_{1}\left(\mathfrak{a}, \mathfrak{b},-\frac{1}{2}+\mathfrak{a}+\mathfrak{b}, 1-\frac{\eta^{2}}{v}\right)\right) \Pi_{\mathbf{k}, 1, \eta,}^{s, t} .
\end{aligned}
$$

In contrast to the equal operator case, there is no unique inverse transform that can extract all Regge moments. The hypergeometric functions compels us to use variations of eq. (3.54) on higher order Regge moments; we leave the study of higher Regge moments for mixed correlators to future work.

\section{Mellin-space functionals}

The position-space dispersion relation in the previous section was inspired by the Mellinspace dispersion relation of eq. (3.13). It is therefore tempting to work directly in Mellinspace by decomposing the Mellin amplitude $M(\mathrm{~s}, \mathrm{t})$ in terms of the absorptive part of the $s$ - and $t$-channel sectors as follows:

$$
\begin{aligned}
& \text { Position-space } \\
& \text { Mellin-space } \\
& \mathcal{G}_{\mathbf{k}}(u, v)=\mathcal{G}_{\mathbf{k}}^{s}(u, v)+\mathcal{G}_{\mathbf{k}}^{t}(u, v) \\
& \leftrightarrow \quad M_{\mathrm{k}}(\mathrm{s}, \mathrm{t})=M_{\mathrm{k}}^{s}(\mathrm{~s}, \mathrm{t})+M_{\mathrm{k}}^{t}(\mathrm{~s}, \mathrm{t}),
\end{aligned}
$$


This is indeed possible and we present the Mellin-representation of the Polyakov-Regge blocks and the $\widehat{B}_{\mathbf{k} ; t \mid m n}^{s, t}$ functionals in this section.

\subsection{Deriving the $\widehat{B}_{\mathrm{k} ; \mathrm{t} \mid m n}^{s, t}$ functional}

The Mellin representation of a correlator $\mathcal{G}(u, v)$ can be written as

$$
\mathcal{G}(u, v)=\iint_{\gamma} \frac{d \mathrm{~s} d \mathrm{t}}{(4 \pi i)^{2}} \Gamma_{\Delta_{i}}^{6}(\mathrm{~s}, \mathrm{t}) u^{\frac{\mathrm{s}-\Delta_{1}-\Delta_{2}}{2}} v^{\frac{\mathrm{t}-\Delta_{2}-\Delta_{3}}{2}} M(\mathrm{~s}, \mathrm{t}),
$$

where s and t are Mellin-Mandelstam variables, $\gamma=\gamma_{\mathrm{s}} \otimes \gamma_{\mathrm{t}}$ is a contour determined by $\Gamma_{\Delta_{i}}^{6}(\mathrm{~s}, \mathrm{t})$ which is defined as

$$
\begin{aligned}
\Gamma_{\Delta_{i}}^{6}(\mathrm{~s}, \mathrm{t})= & \Gamma\left(\frac{\Delta_{1}+\Delta_{2}-\mathrm{s}}{2}\right) \Gamma\left(\frac{\Delta_{3}+\Delta_{4}-\mathrm{s}}{2}\right) \Gamma\left(\frac{\Delta_{2}+\Delta_{3}-\mathrm{t}}{2}\right) \\
& \times \Gamma\left(\frac{\Delta_{1}+\Delta_{4}-\mathrm{t}}{2}\right) \Gamma\left(\frac{\mathrm{s}+\mathrm{t}-\Delta_{1}-\Delta_{3}}{2}\right) \Gamma\left(\frac{\mathrm{s}+\mathrm{t}-\Delta_{2}-\Delta_{4}}{2}\right) .
\end{aligned}
$$

These Mellin-Mandelstam variables are related to the conventional Mellin variables introduced by Mack in [38] as follows:

$$
\begin{aligned}
\gamma_{j j}=-\Delta_{j}, \quad \gamma_{12} & =\frac{\Delta_{1}+\Delta_{2}}{2}-\frac{\mathrm{s}}{2}, \quad \gamma_{13}=\frac{\Delta_{1}+\Delta_{3}}{2}-\frac{\mathrm{u}}{2}, \quad \gamma_{14}=\frac{\Delta_{1}+\Delta_{4}}{2}-\frac{\mathrm{t}}{2}, \\
\mathrm{~s}+\mathrm{t}+\mathrm{u} & =\sum_{i=1}^{4} \Delta_{i} \leftrightarrow \quad \sum_{i} \gamma_{i j}=0 .
\end{aligned}
$$

Using the above, one can write the Mellin-representation of Polyakov-Regge blocks as

$$
\mathcal{P}_{\mathbf{k} ; \Delta, J}^{s, t}(u, v)=\iint_{\gamma} \frac{d \mathrm{~s} d \mathrm{t}}{(4 \pi i)^{2}} \Gamma_{\Delta_{i}}^{6}(\mathrm{~s}, \mathrm{t}) u^{\frac{\mathrm{s}-\Delta_{1}-\Delta_{2}}{2}} v^{\frac{\mathrm{t}-\Delta_{2}-\Delta_{3}}{2}} \mathcal{P}_{\mathbf{k} ; \Delta, J}^{s, t}(\mathrm{~s}, \mathrm{t}) .
$$

The Mellin-space representation of a Polyakov-Regge block is explicitly written in eq. (4.15)-(4.16). The $s$-channel collinear limit corresponds to evaluating the Mellin amplitude at a $s$-channel double-twist value. We thus define the $\widehat{B}_{\mathbf{k} ; t \mid m n}^{s, t}$ Mellin-space functionals as the residue of a Polyakov-Regge block on the $s$-channel pole located at $s=\Delta_{m}+\Delta_{n}+2 k_{m n}$. Singularities from the gamma functions in $\Gamma_{\Delta_{i}}^{6}(\mathrm{~s}, \mathrm{t})$ will cancel zeros from the Mellin-space Polyakov-Regge block to match the OPE expansion. The position-space functionals are then obtained by performing a one-variable inverse Mellin transform: ${ }^{9}$

$$
\begin{aligned}
\widehat{B}_{\mathbf{k} ; \mathrm{t} \mid m n}^{s, t} & =\left.\oint_{\mathrm{s}=\Delta_{m}+\Delta_{n}+2 k_{m n}} \frac{d \mathrm{~s}}{4 \pi i} u^{\frac{\mathrm{s}-\Delta_{1}-\Delta_{2}}{2}} \frac{\mathcal{P}_{\mathbf{k} ; \Delta, J}^{s, t}(\mathrm{~s}, \mathrm{t})}{\Gamma_{\Delta_{i} \mid m n}^{4}(\mathrm{t})}\right|_{u=1}, \\
B_{\mathbf{k} ; v \mid m n}^{s, t} & =\int_{\gamma_{\mathrm{t}} m n} \frac{d \mathrm{t}}{4 \pi i} v^{\frac{\mathrm{t}-\Delta_{2}-\Delta_{3}}{2}} \Gamma_{\Delta_{i} \mid m n}^{4}(\mathrm{t}) \widehat{B}_{\mathbf{k} ; \mathrm{t} \mid m n}^{s, t}
\end{aligned}
$$

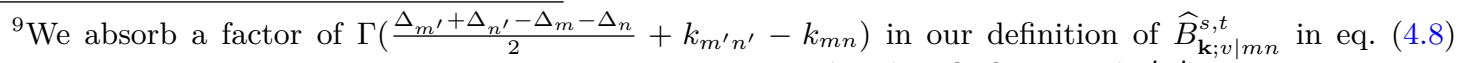
when compared to the equal operator definition given by eq. (3.41) in [31]. Here, $\left(m^{\prime} n^{\prime}\right)$ correspond to the complementary s-channel double-twist family.
} 
where $\gamma_{\mathrm{t}}^{m n}$ denotes the contour integral in t only, and $\Gamma_{\Delta_{i} \mid m n}^{4}(\mathrm{t})$ is a product of four gamma functions reduced from $\Gamma_{\Delta_{i}}^{6}(\mathrm{~s}, \mathrm{t})$ and $P_{\mathbf{k}}$ after evaluating the residue at $\mathrm{s}=\Delta_{m}+\Delta_{n}+2 k_{m n}$ :

$$
\begin{aligned}
\Gamma_{\Delta_{i} \mid m n}^{4}(\mathrm{t})= & \Gamma\left(k_{23}+\frac{\Delta_{2}+\Delta_{3}-\mathrm{t}}{2}\right) \Gamma\left(k_{14}+\frac{\Delta_{1}+\Delta_{4}-\mathrm{t}}{2}\right) \\
& \times \Gamma\left(\frac{\Delta_{m}+\Delta_{n}+2 k_{m n}+\mathrm{t}-\Delta_{1}-\Delta_{3}}{2}\right) \Gamma\left(\frac{\Delta_{m}+\Delta_{n}+2 k_{m n}+\mathrm{t}-\Delta_{2}-\Delta_{4}}{2}\right) .
\end{aligned}
$$

We recall that $(m n)=\{(12),(34)\}$ in our convention. The contour $\gamma_{t}^{m n}$ is determined by $\Gamma_{\Delta_{i} \mid m n}^{4}(\mathrm{t})$ :

$$
\begin{aligned}
\Re\left(\gamma_{\mathrm{t}}^{m n}\right) & =\max \left(-\Delta_{m}-\Delta_{n}-2 k_{m n}+\Delta_{1}+\Delta_{3},-\Delta_{m}-\Delta_{n}-2 k_{m n}+\Delta_{2}+\Delta_{4}\right) \\
& <\Re(\mathrm{t})<\min \left(\Delta_{1}+\Delta_{4}+k_{14}, \Delta_{2}+\Delta_{3}+k_{23}\right), \\
\Im\left(\gamma_{\mathrm{t}}^{m n}\right) & =\Im(\mathrm{t}) \in \mathbb{R} .
\end{aligned}
$$

We find it helpful to explicitely extract the $\Gamma_{\Delta_{i} \mid m n}^{4}(\mathrm{t})$ factor in eq. (4.7) to be consistent with the notation in [30]. The $s$ - and $t$-channel collinear limit thresholds of table 1 fall within the range above. Operators below those threshold are defined by analytic continuation where in Mellin-space, they are poles crossing the contour.

The position-space functionals are computed by deforming the t-contour to the right where it vanishes at the boundary due to boundedness condition in the Regge limit. Therefore, only the lower bound above must be considered carefully. In practice, to obtain the position-space functional $B_{\mathbf{k} ; v \mid 34}^{s, t}$, we integrate eq. (4.8) numerically along the imaginary axis within the range above. The $\gamma_{\mathrm{s}}$ contour can also be determined by $\Gamma_{\Delta_{i}}^{6}$, however we will not need it since we are only interested in computing $B_{\mathbf{k} ; v \mid m n}^{s, t}$ rather than performing the full inverse Mellin transform to obtain the position-space Polyakov-Regge blocks. We will now write explicit formulae for the Mellin-space functional $\widehat{B}_{\mathbf{k} ; t \mid m n}^{s, t}$ appearing in eq. (4.8).

Our starting point will be eq. (3.14) which we re-write here for convenience:

$$
\mathcal{G}_{\mathbf{k}}^{s, t}=\iint_{\gamma} \oint \frac{d \mathrm{~s} d \mathrm{t}}{(4 \pi i)^{2}} \frac{d \mathrm{~s}^{\prime}}{2 \pi i} P_{\mathbf{k}}\left(\mathrm{s}, \mathrm{t} ; \mathrm{s}^{\prime}, \mathrm{t}^{\prime}\right) \Gamma_{\Delta_{i}}^{6}(\mathrm{~s}, \mathrm{t}) u^{\frac{\mathrm{s}-\Delta_{1}-\Delta_{2}}{2}} v^{\frac{\mathrm{t}-\Delta_{2}-\Delta_{3}}{2}} \frac{M^{s, t}\left(\mathrm{~s}^{\prime}, \mathrm{t}^{\prime}\right)}{\mathrm{s}-\mathrm{s}^{\prime}}
$$

where we remind the reader that for fixed $\mathrm{u}$-channel dispersion relation, we have $\mathrm{s}+\mathrm{t}=$ $\mathrm{s}^{\prime}+\mathrm{t}^{\prime}$. Our primary object of interest will be the product of Pochhamer symbols with the Mellin amplitude $P_{\mathbf{k}} \times M^{s, t}\left(\mathrm{~s}^{\prime}, \mathrm{t}^{\prime}\right)$.

The defining feature of Mellin amplitudes is their pole structure: each exchanged operator give rise to an infinite sequence of poles at $\tau_{\mathcal{O}}+2 m$ for $m \in \mathbb{Z}^{0+}$. This statement holds true for both $s$ - and $t$-channel exchanged operators $\mathcal{O}_{s}$ and $\mathcal{O}_{t}$ respectively corresponding to poles at $\mathrm{s}=\tau_{\mathcal{O}_{s}}+2 \mathrm{~m}$ and $\mathrm{t}=\tau_{\mathcal{O}_{t}}+2 \mathrm{~m}$. Moreover, the residue evaluated on these poles is fixed by the OPE:

$$
\lim _{\mathrm{s} \rightarrow \tau_{\mathcal{O}}+2 m} M_{0}(\mathrm{~s}, \mathrm{t}) \sim f_{12 \mathcal{O}} f_{43 \mathcal{O}} \frac{\mathcal{Q}_{\Delta_{\mathcal{O}}, J_{\mathcal{O}}}^{m}(\mathrm{t})}{\mathrm{s}-\tau_{\mathcal{O}}-2 m}
$$

where $\mathcal{Q}_{\Delta, J}^{m}(\mathrm{t})$ are kinematical objects closely related to so-called Mack polynomials [38]. Explicit formulae for unequal external operator Mack polynomials are recorded in appendix $\mathrm{C}$ for convenience. 
Mack polynomials are degree $J$ polynomials with simple zeros at $s$-channel doubletwist values; these zeros coalesce into double-zeros for equal external operators, which are necessary to cancel the double poles from $\Gamma_{\Delta_{i}}^{6}$ in eq. (4.2) in order to produce the correct contribution to the OPE. For mixed correlators, $\Gamma_{\Delta_{i}}^{6}$ has a single pole in each channel which produces a finite result when combined with the zero from the Mack polynomial.

Let us write explicit formulae for the Mellin-space $\widehat{B}_{\mathbf{k} ; t \mid m n}^{s, t}$ functional. We start with the Mellin-space dispersion relation of eq. (3.13) such that we expand the Mellin amplitude in the integrand of eq. (3.14) in terms of both $s$ - and $t$-channel pole contributions:

$$
\begin{aligned}
& M^{s}\left(\mathrm{~s}^{\prime}, \mathrm{t}^{\prime}\right)=\sum_{m=0}^{\infty} \frac{\mathcal{Q}_{\Delta, J}^{m}\left(\mathrm{t}^{\prime}+\mathrm{s}^{\prime}-\tau-2 m-\Delta_{2}-\Delta_{3}\right)}{\mathrm{s}^{\prime}-\tau-2 m} . \\
& M^{t}\left(\mathrm{~s}^{\prime}, \mathrm{t}^{\prime}\right)=\sum_{m=0}^{\infty} \frac{\mathcal{Q}_{\Delta, J}^{m}\left(\mathrm{t}^{\prime}+\mathrm{s}^{\prime}-\tau-2 m-\Delta_{1}-\Delta_{2}\right)}{\mathrm{t}^{\prime}-\tau-2 m} .
\end{aligned}
$$

Polyakov-Regge blocks are then obtained by closing the s' contour towards the boundary to sum over all the contributions of exchanged operators. Since we are working in a fixed-u convention, poles corresponding to operators in the $s$ - and $t$-channels are located at $\mathrm{s}^{\prime}=\tau+2 m$ and $\mathrm{s}^{\prime}=\mathrm{s}+\mathrm{t}-\tau-2 m$ respectively. Formally, we have

$$
\mathcal{P}_{\mathbf{k} ; \Delta, J}^{s}(\mathrm{~s}, \mathrm{t})=\oint_{\mathrm{s}^{\prime}=\tau+2 m} \frac{d \mathrm{~s}^{\prime}}{2 \pi i} P_{\mathbf{k}}\left(\mathrm{s}, \mathrm{t} ; \mathrm{s}^{\prime}, \mathrm{t}^{\prime}\right) \frac{M^{s}\left(\mathrm{~s}^{\prime}, \mathrm{t}^{\prime}\right)}{\mathrm{s}-\mathrm{s}^{\prime}} .
$$

We can further write subtracted Polyakov-Regge blocks in Mellin-space as follows:

$$
\begin{aligned}
& \mathcal{P}_{\mathbf{k} ; \Delta, J}^{s}(\mathrm{~s}, \mathrm{t})=\sum_{m=0}^{\infty} P_{\mathbf{k}}(\mathrm{s}, \mathrm{t} ; \tau+2 m, \mathrm{~s}+\mathrm{t}-\tau-2 m) \frac{\mathcal{Q}_{\Delta, J}^{m}\left(\mathrm{~s}+\mathrm{t}-\tau-2 m-\Delta_{2}-\Delta_{3}\right)}{s-\tau-2 m}, \\
& \mathcal{P}_{\mathbf{k} ; \Delta, J}^{t}(\mathrm{~s}, \mathrm{t})=-\sum_{m=0}^{\infty} P_{\mathbf{k}}(\mathrm{s}, \mathrm{t} ; \mathrm{s}+\mathrm{t}-\tau-2 m, \tau+2 m) \frac{\mathcal{Q}_{\Delta, J}^{m}\left(\mathrm{~s}+\mathrm{t}-\tau-2 m-\Delta_{1}-\Delta_{2}\right)}{\tau+2 m-\mathrm{t}} .
\end{aligned}
$$

The Mellin-space functionals $\widehat{B}_{\mathbf{k} ; t \mid m n}^{s, t}$ are obtained by evaluating the residue of the PolyakovRegge blocks as prescribed by eq. (4.7).

Let us sketch how the Pochhammer sybols $P_{\mathbf{k}}$ and the Mack polynomials conspire to produce vanishing or finite results. The integrand of the $B_{\mathbf{k} ; v \mid m n}^{s}$ functional is given by

$$
B_{\mathbf{k} ; v \mid m n}^{s}=\int_{\gamma_{t}^{m n}} \frac{d \mathrm{t}}{4 \pi i} \oint_{\mathrm{s}=\Delta_{m}+\Delta_{n}+2 k_{m n}} \frac{d \mathrm{~s}}{4 \pi i} \oint_{\mathrm{s}^{\prime}=\tau+2 m} \frac{d \mathrm{~s}}{2 \pi i} \frac{\Gamma_{\Delta_{i}}^{6}(\mathrm{~s}, \mathrm{t}) \mathcal{Q}_{\Delta_{\mathcal{O}}, J_{\mathcal{O}}}^{m}\left(\mathrm{~s}+\mathrm{t}-\Delta_{2}-\Delta_{3}\right)}{\left(\mathrm{s}-\mathrm{s}^{\prime}\right)\left(\mathrm{s}^{\prime}-\tau_{\mathcal{O}}-2 m\right)} P_{\mathbf{k}} \times(\ldots),
$$

where the ellipses are non-singular terms. Let us consider $P_{0}=1$ first. Recall that the Mack polynomials associated with $B_{\mathbf{k} ; v \mid m n}^{s}$ have simple zeros on both $s$-channel trajectories $\Delta_{1}+\Delta_{2}+2 n$ and $\Delta_{3}+\Delta_{4}+2 n$. Evaluating the residue above converts the pole at $\mathrm{s}=\tau+2 m$ into one at $\Delta_{m}+\Delta_{n}+2 k_{m n}=\tau+2 m$. For double-twist operators along the $\tau_{m n} s$ channel trajectory, the simple zero in the Mack polynomial cancels this pole. However, the integrand is absent of poles in the other $s$-channel trajectory $\left(m^{\prime} n^{\prime}\right) \neq(m n)$ and therefore it vanishes in general. Thus, to obtain a finite result for exchanged operators along the 
other $s$-channel trajectory, we can add poles using the ratio of Pochhammer symbols $P_{\mathbf{k}}$. The zero structure described here allows us to decompose any functional $\widehat{B}_{\mathbf{k} ; t \mid m n}^{s, t}$ in terms of double-twist analytic functionals [22-24, 26, 30]:

$$
\left.\widehat{B}_{\mathbf{k} ;|t| m n}^{s}\right|_{\Delta=\Delta_{o}+\ell}=\widehat{a}_{\ell \mid m n, \Delta_{o}}^{\Delta_{i}, s}(\mathrm{t})+\widehat{b}_{\ell \mid m n, \Delta_{o}}^{\Delta_{i}, s}(\mathrm{t})\left(\Delta-\Delta_{o}-\ell\right)+O\left(\left(\Delta-\Delta_{o}-\ell\right)^{2}\right),
$$

where $\Delta_{o}$ takes on double-twist values.

There is a key difference between the equal operator and the mixed correlator cases: equal operator subtracted collinear functionals $B_{k ; v}$ can be expanded into a basis of analytic functionals with double-zeros on double-twists $n>0$. This is no longer the case for mixed correlators unless we restrict ourselves to pairwise equal operators. For the $\langle A A B B\rangle$ and $\langle A B B A\rangle$ correlators, one channel may be expanded into double-zero functional basis elements, while the other channel may be expanded into a basis of functions with simple zeros. We elaborate on this distinguishing feature in appendix D.

\subsection{Mellin-space representation of holographic functionals}

Before ending this section, we derive the Mellin representation of the mixed correlator holographic functional $C_{\mathbf{k} ; \nu \mid 34}^{s, t}$ introduced in section 3.4. To do so, one must substitute the Mellin-space integral representation of $B_{\mathbf{k} ; v \mid 34}^{s, t}$ from eq. (4.8) into eq. (3.57).

Firstly, the $v$-integral which extracts Regge moments can be done straightforwardly using eq. (3.53) by acting on the power of $v$ from the Mellin integral representation of $B_{\mathbf{k} ; v \mid 34}^{s, t}$ :

$$
\begin{aligned}
\int_{\eta^{2}}^{\infty} d v & \frac{\pi^{3 / 2} 2^{1+2\left(\Delta_{2}-\Delta_{4}-k_{12}-k_{14}-k_{23}-k_{34}\right)}\left(v / \eta^{2}\right)^{-\mathfrak{b}} v^{\left(\mathrm{t}-\Delta_{2}-\Delta_{3}\right) / 2}}{\left(v-\eta^{2}\right)^{\frac{1}{2}+\mathfrak{a}+\mathfrak{b}} v^{-\frac{1}{2}\left(\Delta_{2}-\Delta_{4}+k_{12}-k_{14}-k_{23}-k_{34}\right)}} \frac{F_{1}\left(\frac{1}{2}-\mathfrak{b},-\mathfrak{b}, \frac{1}{2}-\mathfrak{a}-\mathfrak{b}, 1-\frac{v}{\eta^{2}}\right)}{\Gamma\left(\frac{1}{2}-\mathfrak{a}-\mathfrak{b}\right) \Gamma(\mathfrak{a}+\mathfrak{b})} \\
= & \frac{2^{-\Delta_{1}+\Delta_{2}-2 \Delta_{4}-3 k_{12}-3 k_{14}-3 k_{23}-k_{34}+\mathrm{t}+3} \pi^{2} \eta^{-\Delta_{1}-\Delta_{2}-k_{12}-k_{14}-k_{23}+k_{34}+\mathfrak{t}+1}}{\Gamma\left(\frac{1}{2}\left(2 k_{12}-2 k_{34}+\Delta_{1}+\Delta_{2}-\Delta_{3}-\Delta_{4}\right)\right)} \\
& \times \frac{\Gamma\left(\Delta_{1}+\Delta_{2}+k_{12}+k_{14}+k_{23}-k_{34}-\mathrm{t}-1\right)}{\Gamma\left(\frac{1}{2}\left(-\mathrm{t}+2 k_{23}+\Delta_{2}+\Delta_{3}\right)\right) \Gamma\left(\frac{1}{2}\left(-\mathrm{t}+2 k_{14}+\Delta_{1}+\Delta_{4}\right)\right)}
\end{aligned}
$$

The $\eta$-integral, which is just a Laplace transform of a Gegenbaueur function, can also be computed analytically; we recast the result from [31] for convenience:

$$
\int_{1}^{\infty}[d \eta] \mathcal{P}_{J}(\eta) \eta^{-X}=\frac{2^{X+d-4} \Gamma\left(\frac{d-1}{2}\right)}{\sqrt{\pi} \Gamma(X)} \Gamma\left(\frac{X+J}{2}\right) \Gamma\left(\frac{X+2-d-J}{2}\right) .
$$

Importantly, $J=\frac{2-d}{2}+i \nu$ such that we obtain a $\gamma_{a}(\nu)$ factor defined by eq. (3.59).

Substituting eq. (4.8) into (3.57), and using the above, we obtain the following result for the Mellin representation of the $C_{\mathbf{k} ; \nu \mid 34}^{s, t}$ functional:

$$
\begin{aligned}
& C_{\mathbf{k} ; \nu \mid 34}^{s, t}=(-1)^{k_{12}+k_{34}+1} 2^{d+2 \Delta_{2}-2 \Delta_{4}-2 k_{12}-2 k_{14}-2 k_{23}-2 k_{34}-1} \frac{a_{\Delta_{i}, k_{i j}}(\nu) \pi^{3 / 2} \Gamma\left(\frac{d-1}{2}\right)}{\Gamma\left(\frac{\Delta_{1}+\Delta_{2}-\Delta_{3}-\Delta_{4}+2 k_{12}-2 k_{34}}{2}\right)} \\
& \times \int_{\tilde{\gamma}_{\mathrm{t}}^{34}} \frac{d \mathrm{t}}{4 \pi i} \Gamma\left(\frac{\mathrm{t}-\Delta_{2}+\Delta_{3}}{2}\right) \Gamma\left(\frac{\mathrm{t}-\Delta_{1}+\Delta_{4}}{2}\right) \gamma_{\Delta_{1}+\Delta_{2}+k_{12}+k_{14}+k_{23}-k_{34}-\mathrm{t}-1}(\nu) \widehat{B}_{\mathbf{k} ; \mathrm{t} \mid 34}^{s, t}
\end{aligned}
$$


This calculation justifies the $\Delta_{i}$ dependence of $\gamma_{a}(\nu)$ in $a_{\Delta_{i}, k_{i j}}(\nu)$ as defined by eq. (3.58): in Mellin-space, the $\gamma_{a}(\nu)$ factor naturally arises to smear the integrand. Furthermore, the $\Delta_{i}$ dependence of $\gamma_{a}(\nu)$ in the integrand is determined by the $s$-channel double-twist families where the functional is evaluated; its arguments would be $\Delta_{3}+\Delta_{4}+k_{34}-k_{12}+k_{23}+k_{14}-1-\mathrm{t}$ for $(m n)=(12)$. To localize the action of the functional in the AdS bulk, we must "unsmear" the integral appropriate $\gamma_{a}(\nu)$ factors. The latter are fixed by the lower bound of the integration region set by $\Gamma_{\Delta_{i} \mid 34}^{4}(\mathrm{t})$ which encodes information from the two u-channel double-twist families. Lastly, the domain of convergence of the integral has changed: there is only a lower bound on the real part of $t$. We leave the study of these mixed correlator holographic functionals for future work.

\section{Applications to the 3D Ising model}

Dispersive functionals have proven to be a valuable tool for the CFT bootstrap program [31, 33]: the suppression of double-twist operators allows one to probe nonperturbative properties of CFTs, while their positivity properties are an enticing feature for the numerical bootstrap. In this section, we extend such explorations by applying our mixed correlator functionals to the 3D Ising model.

In section 5.1, our first goal is to determine the optimal subtraction schemes for each correlator i.e. to determine a sum rule that is balanced by the least number of operators. We will then use this information to derive approximate solutions to crossing for the 3D Ising model in section 5.2.

Below, all computations are evaluated with the collinear functional $B_{\mathbf{k} ; v \mid 34}$, the derivative functional $\left.\partial_{v} B_{\mathbf{k} ; v \mid 34}\right|_{v=1} \equiv B_{\mathbf{k} ; v \mid 34}^{\prime}$, and the $\Phi_{2}$ functional evaluated at $v=1$ where the latter was introduced in [30]. Itt is constructed from a crossing-symmetric subtraction scheme $\mathbf{k}=(1,1,0,0)$ and its features are reviewed in appendix D. Unfortunately, the $\Phi_{2}$ functional becomes computationally expensive at high spin which represents a barrier for high precision measurements (see the discussion for the $\langle\epsilon \epsilon \epsilon \epsilon\rangle$ correlator below). As for the derivative functional, it is best understood in Mellin-space where it is clear that

$$
B_{\mathbf{k} ; v \mid 34}^{\prime s, t}=\int_{\gamma_{\mathrm{t}}^{34}} \frac{d \mathrm{t}}{4 \pi i} \frac{\mathrm{t}-\Delta_{2}-\Delta_{3}}{2} v^{\frac{t-\Delta_{2}-\Delta_{3}}{2}} \Gamma_{\Delta_{i} \mid 34}^{4}(\mathrm{t}) \widehat{B}_{\mathbf{k} ; t \mid 34}^{s, t}
$$

thus it can be viewed as a higher spin Mack polynomial.

One could further use the collinear functional for different values of $v$. We explored the effects of varying $v$ for the $\langle\sigma \sigma \sigma \sigma\rangle$ correlator but found derivative functionals to be more effective; a short discussion about $v \neq 1$ is recorded in appendix E.

We set $k_{34}=0$ to probe the leading double-twist trajectory, and we further restrict ourselves to spin-2 convergent non-log functionals (without using anti-subtractions) in order to include the identity in the OPE. Figures in section 5.1 displaying the action of the functional for varying twist $\tau$ and fixed spin $J$ are normalized with respect to the mean field theory OPE coefficients. Moreover, values shown in the tables in section 5.2 include the OPE coefficients as well. 


\begin{tabular}{|c|c|c|c|c|}
\hline$\left\langle\phi_{A} \phi_{B} \phi_{C} \phi_{D}\right\rangle$ & Functional & Subtraction & Sum rule & Error \\
\hline$\langle\sigma \sigma \sigma \sigma\rangle$ & $B_{\mathbf{k} ; 1 \mid 34}$ & $(1,1,0,0)$ & $\mathbf{1}+\underline{\epsilon}+T_{\mu \nu}-\mathbf{1}_{\mathrm{u}}=0$ & 0.008 \\
& $B_{\mathbf{k} ; 1 \mid 34}^{\prime}$ & $(1,1,0,0)$ & $\mathbf{1}+\underline{\epsilon}+T_{\mu \nu}=0$ & 0.002 \\
& $\Phi_{2}$ & $(1,1,0,0)$ & $\epsilon+T_{\mu \nu}=0$ & 0.06 \\
$\langle\epsilon \sigma \sigma \epsilon\rangle$ & $B_{\mathbf{k} ; 1 \mid 34}$ & $(1,1,0,0)$ & $\underline{\sigma}+\underline{\mathbf{1}+\epsilon}=0$ & -0.03 \\
& $B_{\mathbf{k} ; 1 \mid 34}^{\prime}$ & $(1,1,0,0)$ & $\underline{\sigma}+\mathbf{1}+\epsilon=0$ & 0.09 \\
$\langle\sigma \sigma \epsilon \epsilon\rangle$ & $B_{\mathbf{k} ; 1 \mid 34}$ & $(1,1,0,0)$ & $\underline{\sigma}+\epsilon+T_{\mu \nu}=0$ & -0.004 \\
& $B_{\mathbf{k} ; 1 \mid 34}^{\prime}$ & $(1,1,0,0)$ & $\underline{\sigma}+\epsilon+T_{\mu \nu}=0$ & -0.02 \\
\hline$\langle\epsilon \epsilon \epsilon\rangle$ & $B_{\mathbf{k} ; 1 \mid 34}^{\prime}$ & $(1,1,0,0)$ & $\mathbf{1}+\underline{T_{\mu \nu}}+\epsilon+\epsilon^{\prime}=0$ & 0.06 \\
& $B_{\mathbf{k} ; 1 \mid 34}^{\prime}$ & $(2,0,0,0)$ & $\mathbf{1}+\underline{T}_{\mu \nu}+\epsilon+\underline{\epsilon}^{\prime}=0$ & -0.01 \\
$\langle\epsilon \epsilon \sigma \sigma\rangle$ & $B_{\mathbf{k} ; 1 \mid 34}$ & $(0,1,0,1)$ & $\underline{\sigma}+\mathbf{1}+\epsilon=0$ & -0.03 \\
$\langle\epsilon \sigma \epsilon \sigma\rangle$ & $B_{\mathbf{k} ; 1 \mid 34}$ & $(2,0,0,0)$ & $-\underline{\mathbf{1}_{\mathrm{u}}}+\sigma+[\sigma \epsilon]_{0,2}=0$ & 0.03 \\
\hline
\end{tabular}

Table 4. Most effective truncated sum rules with errors rounded to one significant number based on tabulated results presented later in this section. Errors are normalized with respect to the largest contribution to the sum rule denoted by the underlined operator in the 4th column.

\subsection{Evaluating 3D Ising model sum rules}

To test the accuracy of our functionals, we will evaluate mixed correlator sum rules using the 3D Ising model data provided in [20]. Since dispersive functionals suppress the contribution of double-twist operators, one can hope to truncate the OPE sum to a small subset of operators. We therefore present our results by comparing truncated sums to the total sum of all the following operators where their values are retrieved from [20]: the stable $\mathbb{Z}_{2}$ even and odd operators, and the $[\sigma \sigma]_{0, J},[\epsilon \epsilon]_{0, J},[\sigma \sigma]_{1, J}$ and $[\sigma \epsilon]_{0, J}$ double-twist families. Heavier operators and subleading double-twist families are not considered. We summarize the most effective truncated sum rules in table 4 where we have separate the table into two parts: only sum rules in the first part of the table will be used to derive approximate solutions to crossing in section 5.2, while sum rules in the second part of the table either include new operators $\epsilon^{\prime},[\sigma \epsilon]_{0,2}$, or they are linearly dependent to those in the first part.

In hindsight, it is not surprising that the most effective functionals have crossingsymmetric subtraction schemes: the $s$-channel restriction for non-log functionals is highly constraining for most correlators, and additional $t$-channel subtractions enhances the contribution of low twist operators with twist $\tau<\Delta_{i}+\Delta_{j}+2 k_{i j}-1=\tau^{*}$. Since heavy operators in $s$ - and $t$-channel cancel amongst each other due to the suppression endowed by the dispersive nature of our functionals, only light operators below $\tau^{*}$ dominate the sum rule. Nonetheless, different subtraction schemes lead to functionals with widely different behaviours as shown below and therefore, they might still prove useful as a basis to carve out the space of allowed CFTs. One prominent barrier to such an approach is to construct a positive definite combination of these functionals; it may be possible to construct a mixed correlator matrix with desirable positivity properties as was done in [7]. 


\begin{tabular}{|c|c|c|c|c|c|c|c|c|}
\hline $\mathcal{O}$ & $\mathbb{Z}_{2}$ & $J_{\mathcal{O}}$ & $\tau_{\mathcal{O}}$ & $\Phi_{2}$ & $B_{(1,1,0,0) ; 1 \mid 34}$ & $B_{(2,0,0,0) ; 1 \mid 34}$ & $B_{(1,1,0,0) ; 1 \mid 34}^{\prime}$ & $B_{(2,0,0,0) ; 1 \mid 34}^{\prime}$ \\
\hline$-\mathbf{1}_{\mathrm{u}}$ & + & 0 & 0 & 0 & -0.478 & -0.470 & 0 & 0 \\
\hline 1 & + & 0 & 0 & 0 & -0.478 & -0.470 & 1 & -0.979 \\
\hline$T_{\mu \nu}$ & + & 2 & 1 & 1 & -0.036 & -0.018 & -0.014 & 0.011 \\
\hline$\epsilon$ & + & 0 & 1.412 & -0.944 & 1 & 1 & -0.984 & 1 \\
\hline$\epsilon^{\prime}$ & + & 0 & 3.82968 & & & -0.015 & & -0.016 \\
\hline \multicolumn{4}{|c|}{ Truncated sum } & 0.056 & 0.008 & 0.027 & 0.002 & 0.016 \\
\hline \multicolumn{4}{|c|}{ Sum of all operators } & 0.04 & -0.0008 & 0.01 & 0.0004 & -0.005 \\
\hline
\end{tabular}

Table 5. Contribution of each operator to the $\langle\sigma \sigma \sigma \sigma\rangle$ sum rule with $v=1$; these results include the contribution of the OPE coefficient square $f_{\sigma \sigma \mathcal{O}}^{2}$. The values are normalized with respect to the largest contribution to the sum rule in order to be comparable to the $\Phi_{2}$ sum rule. All other operators contribute to order $O\left(10^{-3}\right)$ and less. Finally, the sum of all operators must add up to 0 in accordance with eq. (5.2).

\subsection{1 $\langle\sigma \sigma \sigma \sigma\rangle$}

We first present sum rules associated with the correlator $\langle\sigma \sigma \sigma \sigma\rangle$. This correlator includes the identity, the $\mathrm{u}$-channel identity and even $\mathbb{Z}_{2}$ operators. Therefore, our sum rule reads

$$
\mathbf{1}-\mathbf{1}_{\mathrm{u}}+\sum_{\mathcal{O} \in \mathbb{Z}_{2}^{+}} f_{\sigma \sigma \mathcal{O}}^{2} \omega[\mathcal{G}]=0
$$

For equal operators, we need at least one $s$-channel subtraction to obtain a non-log functional. Although table 2 might suggest that we require at least one subtraction in the $t$-channel, by evaluating the functional on $\mathcal{G}-\mathbf{1}_{\mathrm{u}}$, we can relax the $t$-channel convergence conditions to allow for $k_{23}=k_{14}=0$. Therefore, we are left with the $\left(k_{12}, k_{23}, k_{34}, k_{14}\right)=(1,1,0,0)$ and $(2,0,0,0)$ subtraction schemes. Numerical results are presented in table 5 .

The $B_{\mathrm{k} ; 1}$ sum rule balances the $\epsilon$ operator against both the u-channel identity and the $s$ - and $t$-channel identity. The $(2,0,0,0)$ subtraction scheme marginally spreads the contribution of all operators across the OPE sum. In contrast, the $\Phi_{2}$ functional mostly balances the stress-tensor against the $\epsilon$ operator; we were able to reproduce table 1 of [30] which we write here again for convenience. We have further included the derivative sum rule which mostly balances the identity against the $\epsilon$ operator. We therefore conclude that different subtraction schemes yield negligeable changes the precision of the $\langle\sigma \sigma \sigma \sigma\rangle$ sum rule.

Let us understand how the values in table 5 are obtained by describing the behaviour of the functionals as a function of twist at fixed spin $J$. Since $s$-channel subtractions convert double zeros into simple zeros, the $(2,0,0,0)$ subtraction scheme is negative definite for $\tau>2 \Delta_{\sigma}+2$ and it is positive between the leading and subleading double-twist i.e. for $2 \Delta_{\sigma}<\tau<2 \Delta_{\sigma}+2$ as shown in figure 4 . We further observe that the absolute value of the $B_{\mathbf{k} \mid 1}$ functional is larger for the $(2,0,0,0)$ subtraction scheme despite the fact that the relative contribution of each operator is similar to the $(1,1,0,0)$ subtraction scheme when normalized with respect to the contribution of the $\epsilon$ operator. This larger action is suggestive of a slower OPE convergence rate which is confirmed by the green and red curves in the left panel of figure 5 . To understand the difference between the $(2,0,0,0)$ and 


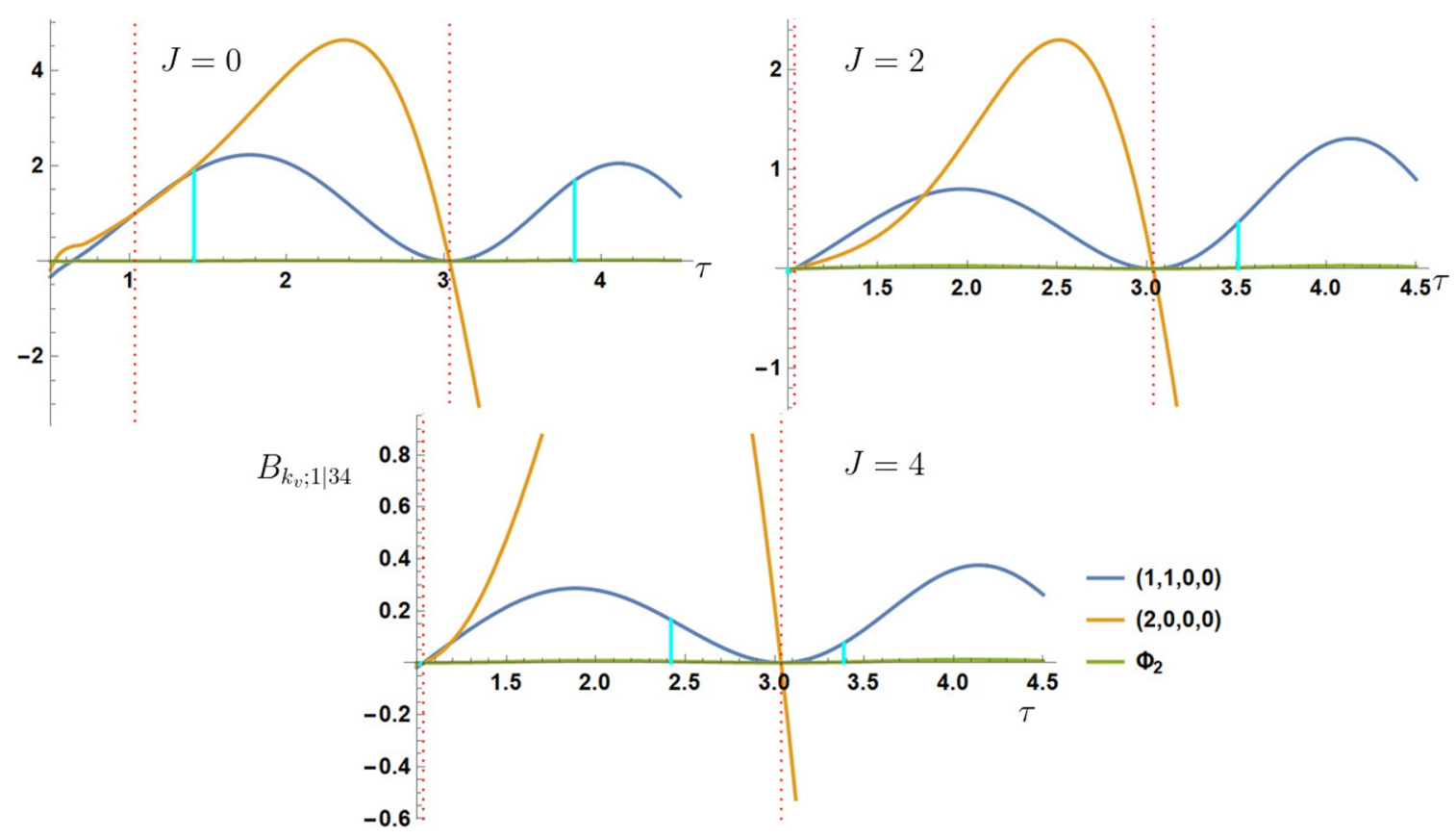

Figure 4. Action of $B_{\mathbf{k} ; 1 \mid 34}$ for the $\langle\sigma \sigma \sigma \sigma\rangle$ correlator acting on conformal blocks $G_{\Delta, J}$ of different spin $J$, twist $\tau=\Delta-J$ and subtraction schemes $\mathbf{k}=(1,1,0,0)$ and $(2,0,0,0)$ represented as blue and orange curves respectively. Red dotted vertical lines denote double-twist values while vertical cyan lines denote the Ising model operators appearing in the OPE of $\langle\sigma \sigma \sigma \sigma\rangle$.

$(1,1,0,0)$ subtraction schemes at large twist, let us analyze the mellin-space functionals:

$$
\begin{aligned}
& \widehat{B}_{(1,1,0,0) ; \mathrm{t}} \propto\left(2 \Delta_{\phi}-\mathrm{t}\right), \\
& \widehat{B}_{(2,0,0,0) ; \mathrm{t}} \propto \frac{(\Delta-J)^{2}+4 \Delta_{\phi}^{2}-2 \Delta_{\phi}(\Delta+\mathrm{t}-2-J)+\mathrm{t}^{2}-(\Delta+2-J) \mathrm{t}}{\left(-\Delta+2 \Delta_{\phi}+2 J-2\right)(-\Delta+\mathrm{t}+J-2)} .
\end{aligned}
$$

We note the $\Delta$ and t dependence in the above which enhances the action of the positionspace functional for larger twist. From this example, we learn that an ideal subtraction scheme would suppress the contribution of larger twist operators for fixed spin $J$ relative to the low twist sector.

\subsection{2 $\langle\epsilon \epsilon \epsilon \epsilon\rangle$}

One could also apply these functionals on the $\langle\epsilon \epsilon \epsilon \epsilon\rangle$ correlator. However, the OPE sum converges more slowly than for the $\langle\sigma \sigma \sigma \sigma\rangle$ correlators as illustrated in figure 5. Indeed, by comparing the red and green curves to the blue and orange ones, large spin operators are necessary to provide convergence and therefore, one cannot truncate the data as effectively as was done for the $\langle\sigma \sigma \sigma \sigma\rangle$ correlator. This leads to slowly convergent sum rules as shown in table 6 .

This slow convergence can be better understood from figure 6 . We see that both $[\sigma \sigma]_{0, J}$ and $[\epsilon \epsilon]_{0, J}$ families are negative due to their twists falling below the collinear thresholds tabulated in table 1 such that they are defined by analytic continuation (by shifting the Mellin-mandelstam $t$ contour). Therefore, the slow convergence can be remedied by adding 

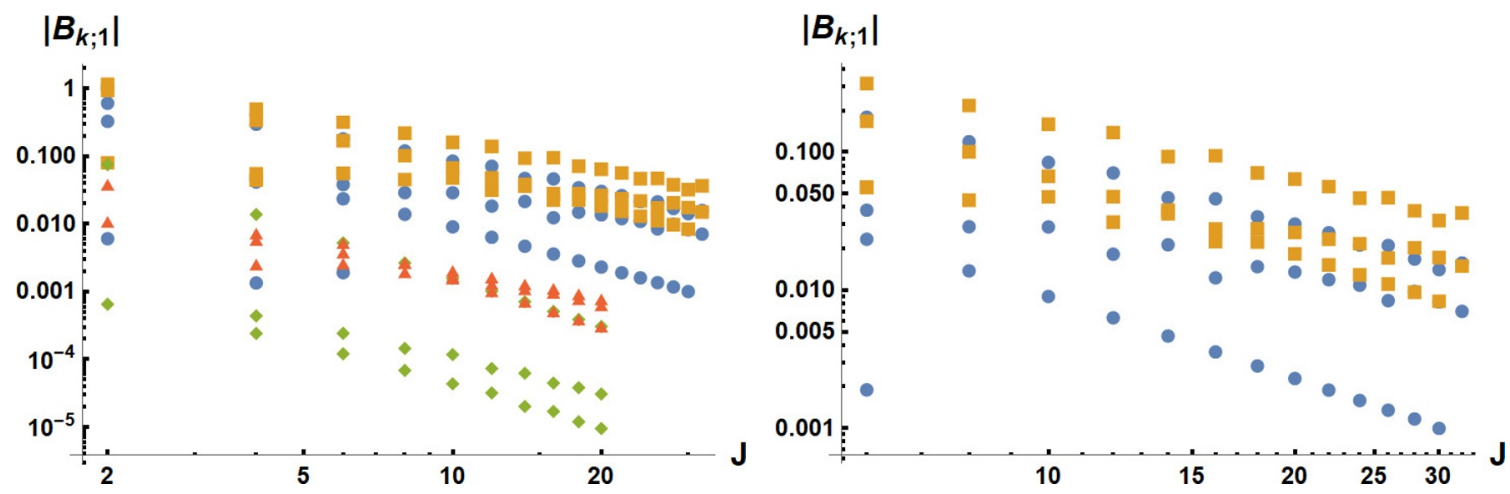

Figure 5. We plot the absolute value of the functional contribution for various operators as a function of spin. The green and red plots correspond to the $B_{(1,1,0,0) ; 1}$ and $B_{(2,0,0,0) ; 1}$ functionals applied to the $\langle\sigma \sigma \sigma \sigma\rangle$ correlator. The blue and orange plots correspond to the $B_{(1,1,0,0) ; 1}$ and $B_{(2,0,0,0) ; 1}$ functionals applied to the $\langle\epsilon \epsilon \epsilon \epsilon\rangle$ correlator respectively. The right panel is a close-up of the $B_{\mathbf{k} ; 1}$ functionals applied to the $\langle\epsilon \epsilon \epsilon \epsilon\rangle$ correlator. In the right panel, one double-twist family converging more quickly for the $(1,1,0,0)$ subtraction scheme than others: this is the $[\sigma \sigma]_{0, J}$ family.

heavier operators in our sum. This is only true of the $\mathbf{k}=(1,1,0,0)$ subtraction scheme where heavier higher twist operators would contribute positively to the sum to support the contributions from the $[\sigma \sigma]_{1, J}$ against lower twist operator families $[\sigma \sigma]_{0, J}$ and $[\epsilon \epsilon]_{0, J}$. On the other hand, the $(2,0,0,0)$ subtracted functional converges well since it mostly balances the $[\sigma \sigma]_{1, J}$ family against all other operators. As for the $\Phi_{2}$ functional, the sum rule appears to be non-convergent ${ }^{10}$ as shown in figure 5 . This non-convergence implies that the swappability property is lost. A rigorous understanding of this observations requires a large spin analysis which is more difficult to perform in Mellin-space (see [32]) than in positionspace. It would be interesting to further test convergence properties of the $\Phi_{2}$ functional at large spin by obtaining a position-space representation of this projected functional.

The slow convergence prevents any reasonable truncation of the OPE as shown by the results in table 6 . The derivative sum rule $B_{\mathbf{k} ; 1 \mid 34}^{\prime}$ on the other hand is dominated by the identity, the stress tensor, $\epsilon$ and $\epsilon^{\prime}$. The presence of the $\epsilon^{\prime}$ operator prevents us from using these sum rules to derive partial sums satisfying crossing in the next section, however they are a good candidate to be used in future bootstrap computations. The improved convergence for the derivative functional can be understood from eq. (5.1): higher powers in $\mathrm{t}$ can be viewed as higher spin Mack polynomials which translates into an accelerated convergence in spin. Lastly, we record the action of $\Phi_{2}$ and $B_{\mathbf{k} ; 1 \mid 34}$ on the $\langle\epsilon \epsilon \epsilon \epsilon\rangle$ correlator for spins $J=0,2,4$ in figure 7 .

\footnotetext{
${ }^{10}$ The original contour for $\Phi_{2}$ runs along the entire imaginary axis at $\Re(\mathrm{t})=\Delta_{\phi}$. For operators with $\tau+2 m<\Delta_{\phi}$ such as operators in the $[\sigma \sigma]_{0, \ell}$ family, a logarithmic branch point appears to the left of the first pole originating from $\Gamma_{\Delta_{\phi}}^{4}(\mathrm{t})$. We therefore deform the contour to the left between $0<\Re(\mathrm{t})<\tau+2 m$ such that the integral wraps the branch cut which extends to the right. The $\Phi_{2}$ functional's integrand vanishes along this new contour as $\mathrm{t} \rightarrow \pm i \infty$.
} 


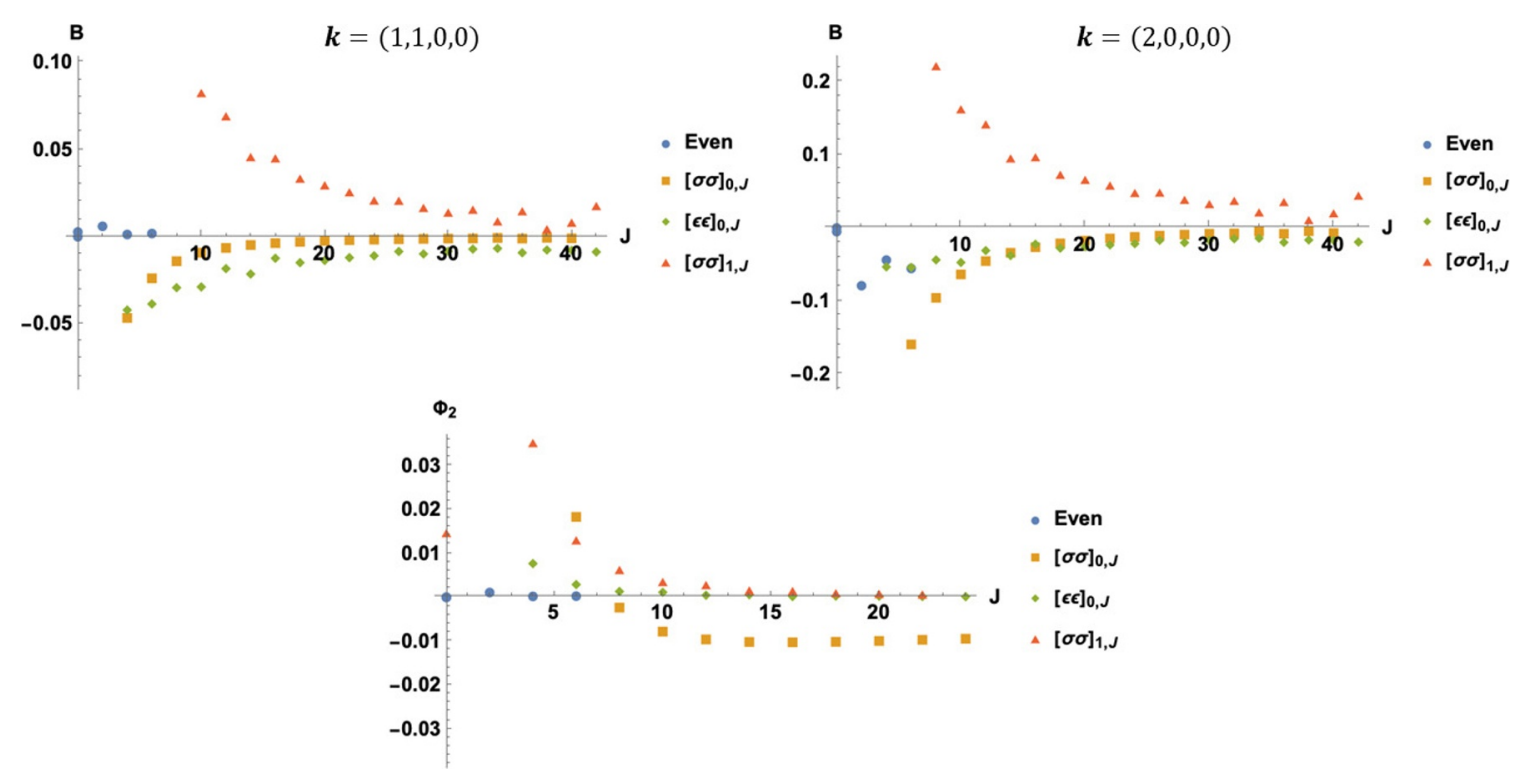

Figure 6. Action of the $B_{\mathbf{k} ; 1}$ and $\Phi_{2}$ functional on exchanged operators in the OPE of the $\langle\epsilon \epsilon \epsilon \epsilon$ correlator. The "even" family in the legend corresponds to other stable $\mathbb{Z}_{2}$ even operators. The functional acting on the $[\sigma \sigma]_{0, J}$ and low twist $[\epsilon \epsilon]_{0, J}$ families are defined by analytic continuation. For the $\Phi_{2}$ functional, the projection lifts the negativity of the $[\epsilon \epsilon]_{0, J}$ family at the cost of losing convergence of the $[\sigma \sigma]_{0, J}$ family for higher spin operators.

\begin{tabular}{|c|c|c|c|c|c|c|c|c|}
\hline $\mathcal{O}$ & $\mathbb{Z}_{2}$ & $J_{\mathcal{O}}$ & $\tau_{\mathcal{O}}$ & $\Phi_{2}$ & $B_{(1,1,0,0) ; 1 \mid 34}$ & $B_{(2,0,0,0) ; 1 \mid 34}$ & $B_{(1,1,0,0) ; 1 \mid 34}^{\prime}$ & $B_{(2,0,0,0) ; 1 \mid 34}^{\prime}$ \\
\hline$-\mathbf{1}_{\mathrm{u}}$ & + & 0 & 0 & 0 & -0.731 & -0.379 & 0 & 0 \\
$\mathbf{1}$ & + & 0 & 0 & 0 & -0.731 & -0.379 & -0.692 & -0.755 \\
$T_{\mu \nu}$ & + & 2 & 1 & -1 & 0.238 & -0.433 & 1 & 0.269 \\
$\epsilon$ & + & 0 & 1.41263 & 0.137 & -0.820 & -0.271 & -0.697 & -0.527 \\
$\epsilon^{\prime}$ & + & 0 & 3.82968 & & 1 & 1 & 0.447 & 1 \\
{$[\sigma \sigma]_{0, J}$} & + & 4 & 1.02267 & 0.206 & & -0.120 & & \\
{$[\sigma \sigma]_{1, J}$} & + & 2 & 3.50915 & 0.150 & 0.449 & 0.337 & & \\
{$[\sigma \sigma]_{1, J}$} & + & 4 & 3.38568 & & 0.212 & 0.185 & & \\
{$[\sigma \sigma]_{1, J}$} & + & 6 & 3.32032 & & 0.127 & 0.120 & & \\
\hline \multicolumn{2}{|l}{ Sum of all operators } & -0.475 & -0.139 & 0.05 & 0.0004 & -0.005 \\
\hline
\end{tabular}

Table 6. Contribution of each operator to the $\langle\epsilon \epsilon \epsilon \epsilon\rangle$ sum rule with $v=1$; these results include the contribution of the OPE coefficient square $f_{\epsilon \epsilon \mathcal{O}}^{2}$. Empty cells and all other operators contribute at order $O\left(10^{-2}\right)$ and smaller. Finally, the sum of all operators must add up to 0 in accordance with eq. (5.2).

\subsection{3 $\langle\epsilon \sigma \epsilon \sigma\rangle$}

Since the $\langle\epsilon \sigma \epsilon \sigma\rangle$ correlator is manifestly s $\leftrightarrow$ t-channel crossing-symmetric, the sum rule reads:

$$
-\mathbf{1}_{\mathrm{u}}+\sum_{\mathcal{O} \in \mathbb{Z}_{2}^{-}}(-1)^{J} f_{\epsilon \sigma \mathcal{O}}^{2} \omega[\mathcal{G}]=0 .
$$

For this first mixed correlator, as indicated in table 2, we require at least one $s$-channel subtraction to obtain non-log functionals while subtractions in the $t$-channel are uncon- 


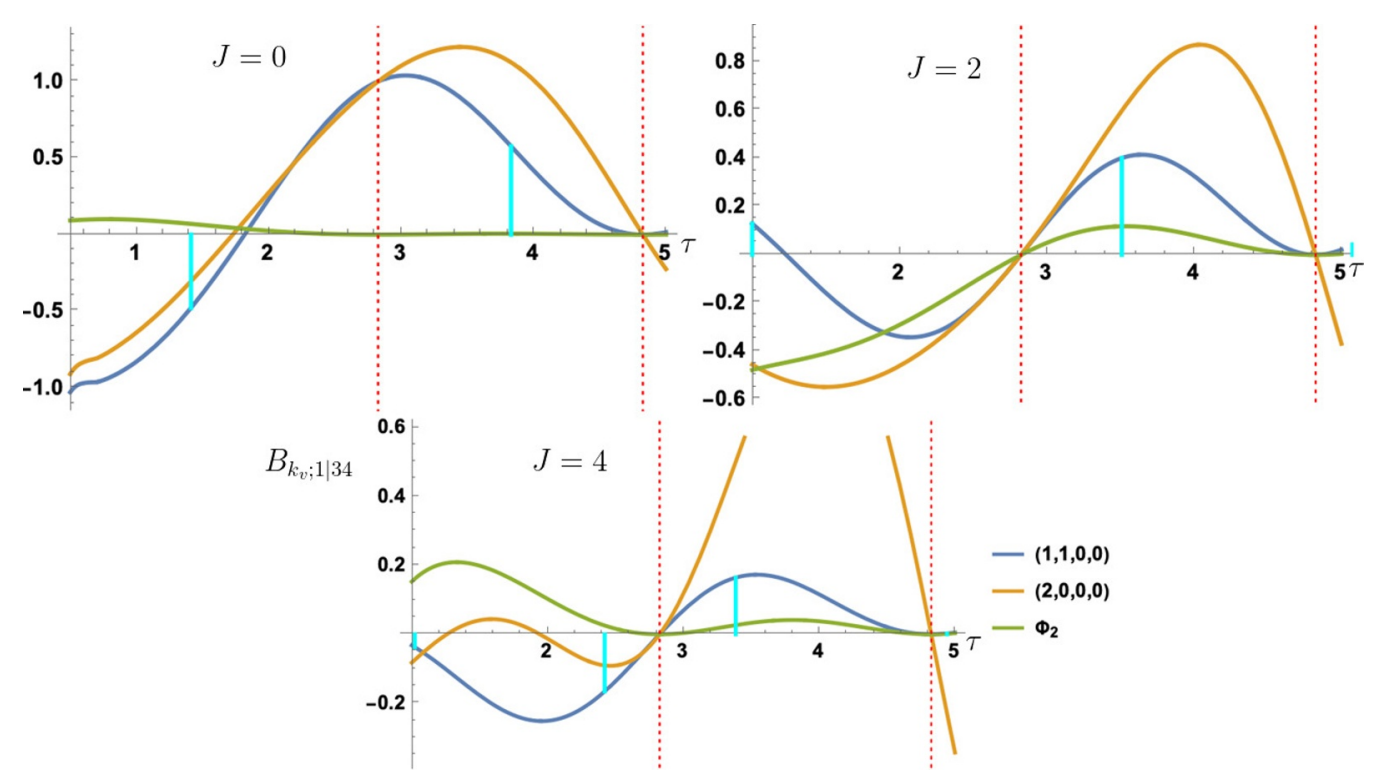

Figure 7. Action of $B_{\mathbf{k} ; 1 \mid 34}$ for the $\langle\epsilon \epsilon \epsilon \epsilon\rangle$ acting on conformal blocks $G_{\Delta, J}$ of different spin $J$, twist $\tau=\Delta-J$ and subtraction schemes $\mathbf{k}=(1,1,0,0)$ and $(2,0,0,0)$ represented as blue and orange curves respectively. Red dotted vertical lines denote double-twist values while vertical cyan lines denote the Ising model operators appearing in the OPE of $\langle\epsilon \epsilon \epsilon \epsilon\rangle$.

\begin{tabular}{|c|c|c|c|c|c|c|c|}
\hline $\mathcal{O}$ & $\mathbb{Z}_{2}$ & $J$ & $\tau_{\mathcal{O}}$ & $B_{(2,0,0,0) ; 1 \mid 34}$ & $B_{(1,1,0,0) ; 1 \mid 34}$ & $B_{(2,0,0,0) ; 1 \mid 34}^{\prime}$ & $B_{(1,1,0,0) ; 1 \mid 34}^{\prime}$ \\
\hline$-\mathbf{1}_{\mathrm{u}}$ & + & 0 & 0 & -1 & -1 & 0 & 0 \\
\hline$\sigma$ & - & 0 & 0.518149 & 0.865 & 0.608 & 1 & -0.892 \\
\hline$[\sigma \epsilon]_{0, J}$ & - & 2 & 2.18031 & 0.162 & 0.295 & -0.676 & 1 \\
\hline$[\sigma \epsilon]_{0, J}$ & - & 3 & 1.63804 & -0.038 & -0.077 & 0.042 & -0.207 \\
\hline$[\sigma \epsilon]_{0, J}$ & - & 4 & 2.11267 & 0.067 & 0.111 & -0.056 & 0.130 \\
\hline \multirow{2}{*}{\multicolumn{4}{|c|}{ Truncated sum }} & 0.057 & -0.062 & 0.314 & 0.031 \\
\hline & & & & 0.07 & -0.03 & -0.2 & -0.005 \\
\hline
\end{tabular}

Table 7. Contribution of each operator to the $\langle\epsilon \sigma \epsilon \sigma\rangle$ sum rule with $v=1$ normalized relative to the largest contributor of the sum rule; these results include the contribution of the OPE coefficient square $(-1)^{J} f_{\epsilon \sigma \mathcal{O}}^{2}$. Sum rules from these subtracted functionals should add up to 0 in accordance with eq. (5.4). Other operators contribute at order $O\left(10^{-2}\right)$ and smaller; we show the $[\sigma \epsilon]_{0,3}$ operator to illustrate the negativity for odd spin operators in these sum rules.

strained. Moreover, the two $t$-channel trajectories are indistinguishable. and therefore, we present results for the following $\left(k_{12}, k_{23}, k_{34}, k_{14}\right)$ subtraction schemes in table $7:(2,0,0,0)$ and $(1,1,0,0)$.

From table 7, we first note that odd spin operators have negative contributions while even spin operators contribute positively in accordance with eq. (5.4). That being said, the pair of operators with spin $J_{o d d}$ and $J_{o d d}+1$ always act positively in order to balance the contribution of the $\mathrm{u}$-channel identity. Moreover, the sum rule associated with the derivative $B_{(2,0,0,0) ; 1 \mid 34}^{\prime}$ functional does not satisfy eq. (5.4). According to figure 8 , the large spin tail is convergent and therefore we conclude that this functional is sensitive 

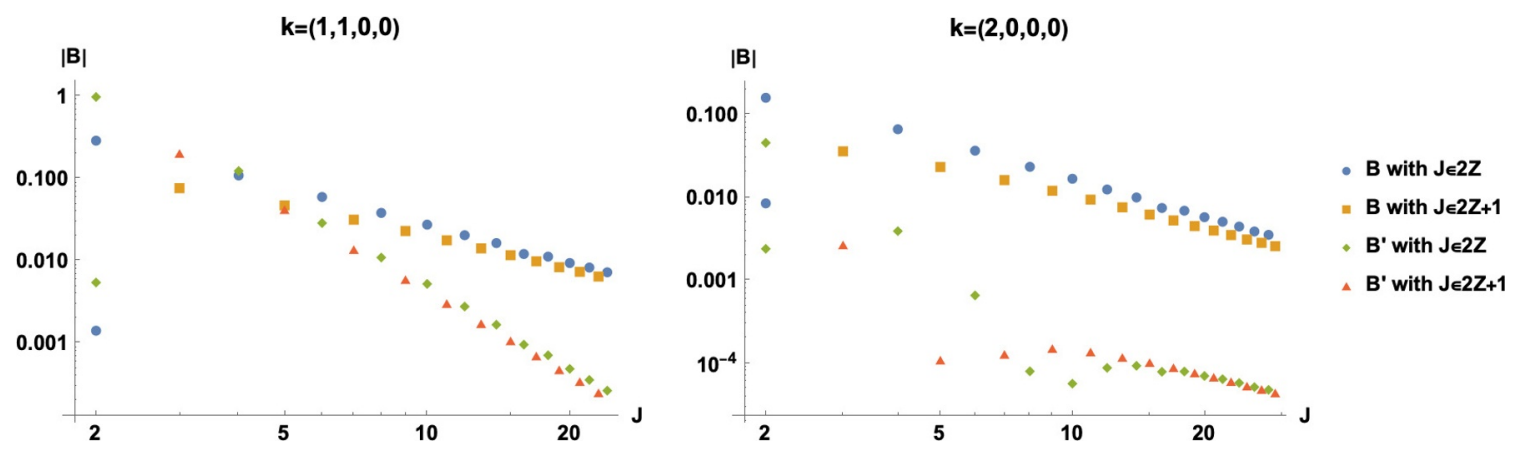

Figure 8. Log-log plot of the absolute value of the collinear $B_{\mathbf{k} ; 1 \mid 34}$ and derivative $B_{\mathbf{k} ; 1 \mid 34}^{\prime}$ functionals acting on exchanged operators of the $\langle\epsilon \sigma \epsilon \sigma\rangle$ correlator. The left and right plots corresponds to the $\mathbf{k}=(1,1,0,0)$ and $\mathbf{k}=(2,0,0,0)$ subtraction schemes respectively.
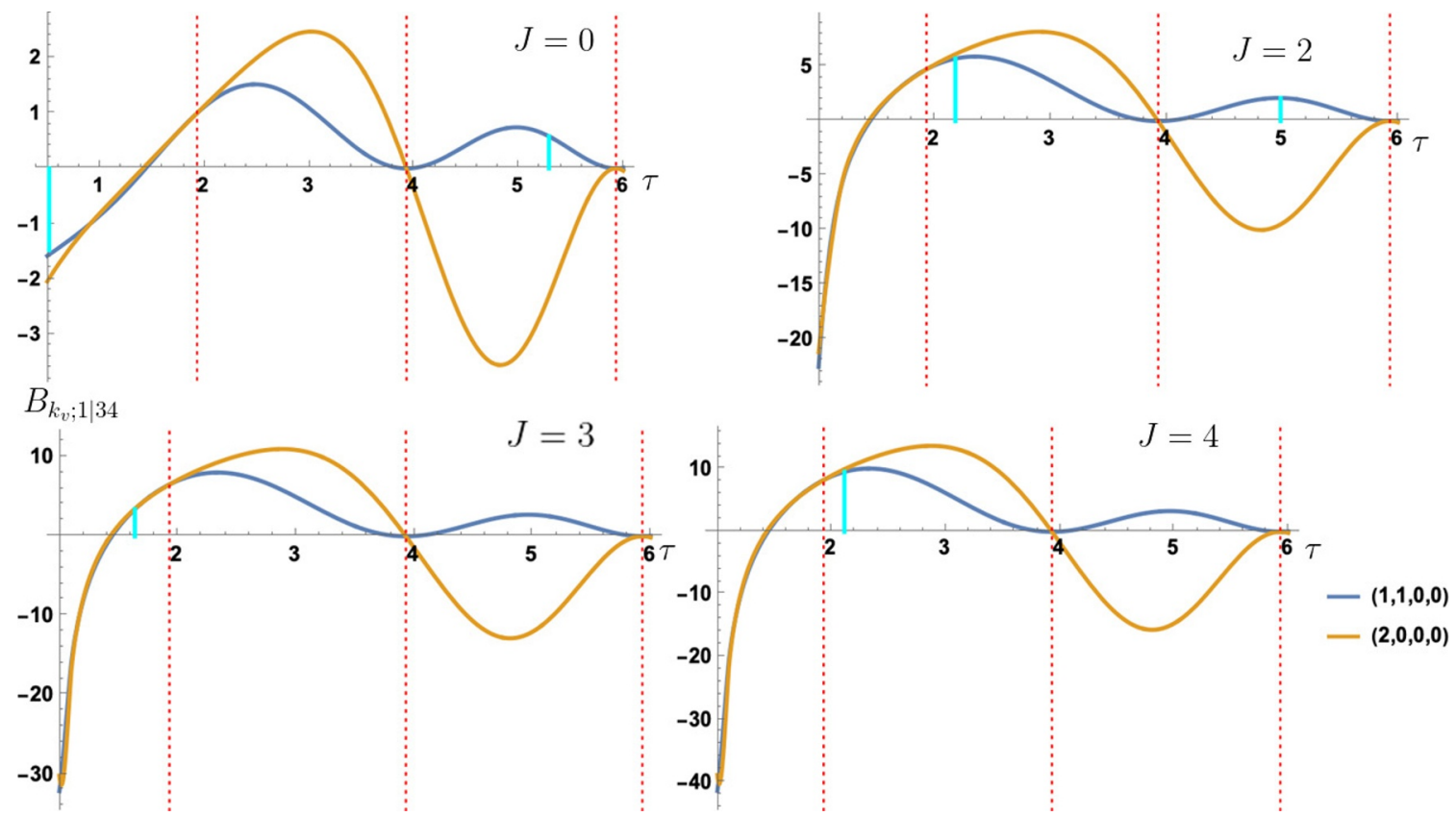

Figure 9. Action of $B_{\mathbf{k} ; 1 \mid 34}$ for the $\langle\epsilon \sigma \epsilon \sigma\rangle$ acting on conformal blocks $G_{\Delta, J}$ of different spin $J$, twist $\tau=\Delta-J$ and subtraction schemes $\mathbf{k}=(1,1,0,0)$ and $(2,0,0,0)$ represented as blue and orange curves respectively. Red dotted vertical lines denote double-twist values while vertical cyan lines denote the Ising model operators appearing in the OPE of $\langle\epsilon \sigma \epsilon \sigma\rangle$.

to heavier operators not included in the OPE sum. Figure 9 shows the action of the $f_{\epsilon \sigma \mathcal{O}}^{2} B_{\mathbf{k} ; 1 \mid 34}$ functional for the $\langle\epsilon \sigma \epsilon \sigma\rangle$ correlator.

\subsection{4 $\langle\epsilon \sigma \sigma \epsilon\rangle$}

We now explore dispersive sum rules associated with the $\langle A B B A\rangle$ correlator:

$$
\sum_{\mathcal{O} \in \mathbb{Z}_{2}^{+}} f_{\sigma \sigma \mathcal{O}} f_{\epsilon \epsilon \mathcal{O}} B_{\mathcal{O}}^{t}+\sum_{\mathcal{P} \in \mathbb{Z}_{2}^{-}} f_{\epsilon \sigma \mathcal{P}}^{2} B_{\mathcal{P}}^{s}=0 .
$$




\begin{tabular}{|c|c|c|c|c|c|}
\hline $\mathcal{O}$ & $\mathbb{Z}_{2}$ & $J$ & $\tau_{\mathcal{O}}$ & $B_{(1,1,0,0) ; 1 \mid 34}$ & $B_{(1,1,0,0) ; 1 \mid 34}^{\prime}$ \\
\hline$\sigma$ & - & 0 & 0.518149 & 1 & 1 \\
\hline$[\sigma \epsilon]_{0, J}$ & - & 2 & 2.18031 & 0.027 & -0.089 \\
\hline 1 & + & 0 & 0 & -0.465 & -0.628 \\
\hline$\epsilon$ & + & 0 & 1.41263 & -0.563 & -0.280 \\
\hline$T_{\mu \nu}$ & + & 2 & 3 & -0.011 & 0.042 \\
\hline \multicolumn{4}{|c|}{$\sigma+\mathbf{1}+\epsilon$} & -0.028 & 0.092 \\
\hline \multicolumn{4}{|c|}{ Sum of all operators $B^{s}+B^{t}$} & -0.002 & 0.005 \\
\hline
\end{tabular}

Table 8. Contribution of each operator to the $\langle\epsilon \sigma \sigma \epsilon\rangle$ sum rule with $v=1$; these results include

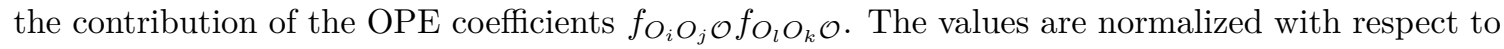
the largest contribution to the sum rule i.e. the $\sigma$ operator. All other operators contribute at order $O\left(10^{-3}\right)$ and less.

Since double-twist operators are exchanged in the $s$-channel, we require at least one $s$ channel subtraction to obtain non-log functionals. In the $t$-channel, depending on the operator ordering, we need at least one subtraction along one of the $t$-channel twist trajectory. Therefore, WLOG, we study the $\langle\epsilon \sigma \sigma \epsilon\rangle$ correlator. Given this operator ordering and the conditions in table 2 , we are left with a single allowable subtraction scheme: $\mathbf{k}=\left(k_{12}, k_{23}, k_{34}, k_{14}\right)=(1,1,0,0)$. Contributions to this sum rule is recorded in table 8.

Due to the two double-twist families $[\sigma \sigma]_{n, J}$ and $[\epsilon \epsilon]_{n, J}$, the $t$-channel collinear functional is not guaranteed to be sign-definite. However, this only occurs for $2 \Delta_{\sigma}+2 \geq \tau \geq 2 \Delta_{\epsilon}$ where no operators are exchanged. Moreover, the subtraction scheme guarantees that the functional possess the same sign for $t$-channel operators outside that rnage. Therefore, the sum rule is satisfied by balancing sign-definite contributions from $s$-channel operators against sign-definite contribution from the $t$-channel separately. This feature will not be preserved for the $\langle A A B B\rangle$ correlator. The action of the functional for varying twist at fixed spin is plotted in figure 10 and 11 along with the next correlators.

According to table 8, this sum rule mostly balances the $\sigma$ operator against the identity and the $\epsilon$ operator. The derivative sum rule enhances the identity contribution at the cost of lowering the contribution of the $\epsilon$ operator.

\subsection{5 $\langle\sigma \sigma \epsilon \epsilon\rangle$ and $\langle\epsilon \epsilon \sigma \sigma\rangle$}

Finally, we examine correlators of the form $\langle A A B B\rangle$. The sum rule for this correlator is the same as that of eq. (5.5), but with $B^{t} \leftrightarrow B^{s}$ exchanged. However, significant differences follow from the discussion about convergence in section 3.3.

Based on table 2 , it is preferable to set $\left(\Delta_{A}, \Delta_{B}\right)=\left(\Delta_{\sigma}, \Delta_{\epsilon}\right)$ i.e. to study the $\langle\sigma \sigma \epsilon \epsilon\rangle$ correlator; this ordering minimizes the number of necessary $t$-channel subtractions by requiring that $k_{23}, k_{14}>-0.447$ whereas the other operator ordering would require that $k_{23}, k_{14}>0.447$. Furthermore, the two $t$-channel subtractions are indistiguishable, while $s$-channel subtractions are unconstrained even for non-log functionals. We therefore detail the action of the $B_{\mathbf{k} ; v \mid 34}$ functional with subtraction schemes $(2,0,0,0),(0,1,0,1),(1,1,0,0)$ 


\begin{tabular}{|c|c|c|c|c|c|c|c|}
\hline $\mathcal{O}$ & $\mathbb{Z}_{2}$ & $J$ & $\tau_{\mathcal{O}}$ & $B_{(2,0,0,0) ; 1 \mid 34}$ & $B_{(0,1,0,1) ; 1 \mid 34}$ & $B_{(1,1,0,0) ; 1 \mid 34}$ & $B_{(0,2,0,0) ; 1 \mid 34}$ \\
\hline$\sigma$ & - & 0 & 0.518149 & 1 & -0.311 & 1 & -0.593 \\
\hline$[\sigma \epsilon]_{0, J}$ & - & 2 & 2.18031 & & -0.207 & & -0.224 \\
\hline 1 & + & 0 & 0 & 0 & 1 & 0 & 0 \\
\hline$\epsilon$ & + & 0 & 1.41263 & -0.621 & -0.496 & -0.586 & 1 \\
\hline$T_{\mu \nu}$ & + & 2 & 3 & -0.226 & & -0.418 & \\
\hline$\epsilon^{\prime}$ & + & 0 & 3.82968 & -0.115 & & & -0.111 \\
\hline \multirow{2}{*}{\multicolumn{4}{|c|}{$\begin{array}{c}\text { Truncated sum } \\
\text { Sum of all operators } B^{s}+B^{t}\end{array}$}} & 0.038 & -0.014 & 0.006 & 0.072 \\
\hline & & & & -0.05 & 0.0003 & 0.0003 & 0.005 \\
\hline
\end{tabular}

Table 9. Contribution of each operator to the $B$ functional acting on the $\langle\sigma \sigma \epsilon \epsilon\rangle$ correlator with $v=1$; these results include the contribution of the OPE coefficients $f_{O_{i} O_{j} \mathcal{O}} f_{O_{l} O_{k} \mathcal{O}}$. The values are presented as ratios of the individual operator relative to the largest contribution to the sum rule. Empty cells contribute at order $O\left(10^{-2}\right)$ and less.

\begin{tabular}{|c|c|c|c|c|c|c|c|}
\hline $\mathcal{O}$ & $\mathbb{Z}_{2}$ & $J$ & $\tau_{\mathcal{O}}$ & $B_{(2,0,0,0) ; 1 \mid 34}^{\prime}$ & $B_{(0,1,0,1) ; 1 \mid 34)}^{\prime}$ & $B_{(1,1,0,0) ; 1 \mid 34}^{\prime}$ & $B_{(0,2,0,0) ; 1 \mid 34}^{\prime}$ \\
\hline$\sigma$ & - & 0 & 0.518149 & 1 & -0.116 & 1 & -0.322 \\
\hline$[\sigma \epsilon]_{0, J}$ & - & 2 & 2.18031 & & -0.412 & & -0.586 \\
\hline$[\sigma \epsilon]_{0, J}$ & - & 3 & 1.63804 & & -0.169 & & 0.191 \\
\hline 1 & + & 0 & 0 & 0 & 1 & 0 & 0 \\
\hline$\epsilon$ & + & 0 & 1.41263 & -0.268 & -0.358 & -0.296 & 1 \\
\hline$T_{\mu \nu}$ & + & 2 & 3 & -0.695 & & -0.719 & -0.231 \\
\hline \multirow{2}{*}{\multicolumn{4}{|c|}{$\begin{array}{c}\text { Truncated sum } \\
\text { Sum of all operators } B^{s}+B^{t}\end{array}$}} & 0.037 & -0.055 & -0.015 & 0.052 \\
\hline & & & & 0.008 & 0.003 & 0.00007 & -0.005 \\
\hline
\end{tabular}

Table 10. Contribution of each operator to the derivative $B^{\prime}$ functional acting on the $\langle\sigma \sigma \epsilon \epsilon\rangle$ correlator with $v=1$; these results include the contribution of the OPE coefficients $f_{O_{i} O_{j} \mathcal{O}} f_{O_{l} O_{k} \mathcal{O}}$. The values are presented as ratios of the individual operator relative to the largest contribution to the sum rule. Empty cells contribute at order $O\left(10^{-2}\right)$ and less.

and $(0,2,0,0)$ for the $\langle\sigma \sigma \epsilon \epsilon\rangle$ correlator in the table 9 . We present the action of the derivative functional $B_{\mathbf{k} ; v \mid 34}^{\prime}$ in table 10 .

One could also consider the $\langle\epsilon \epsilon \sigma \sigma\rangle$ correlator which would require a subtraction in both $t$-channel trajectories thereby restricting the space of twice-subtracted functionals to the $\mathbf{k}=(0,1,0,1)$ subtraction scheme; we present numerical results for this sum rule in table 11 .

To better understand the distinguishing features of these functionals, we plot their action as a function of twist for fixed spins in figure 10 and 11. We highlight four features of these figures: their sign, the largest contributor to the $\langle\sigma \sigma \epsilon \epsilon\rangle$ correlator, the presence or absence of the identity exchange, and the difference between the $\langle\sigma \sigma \epsilon \epsilon\rangle$ and $\langle\epsilon \epsilon \sigma \sigma\rangle$ correlator with $(0,1,0,1)$ subtraction scheme.

Let us first discuss the sign of these functionals. The latter returns simple zeros on the two $s$-channel double-twist families. By evaluating the functional on the $(m n)$ double-twist family, the funcitonal becomes non-zero on the corresponding double-twist 

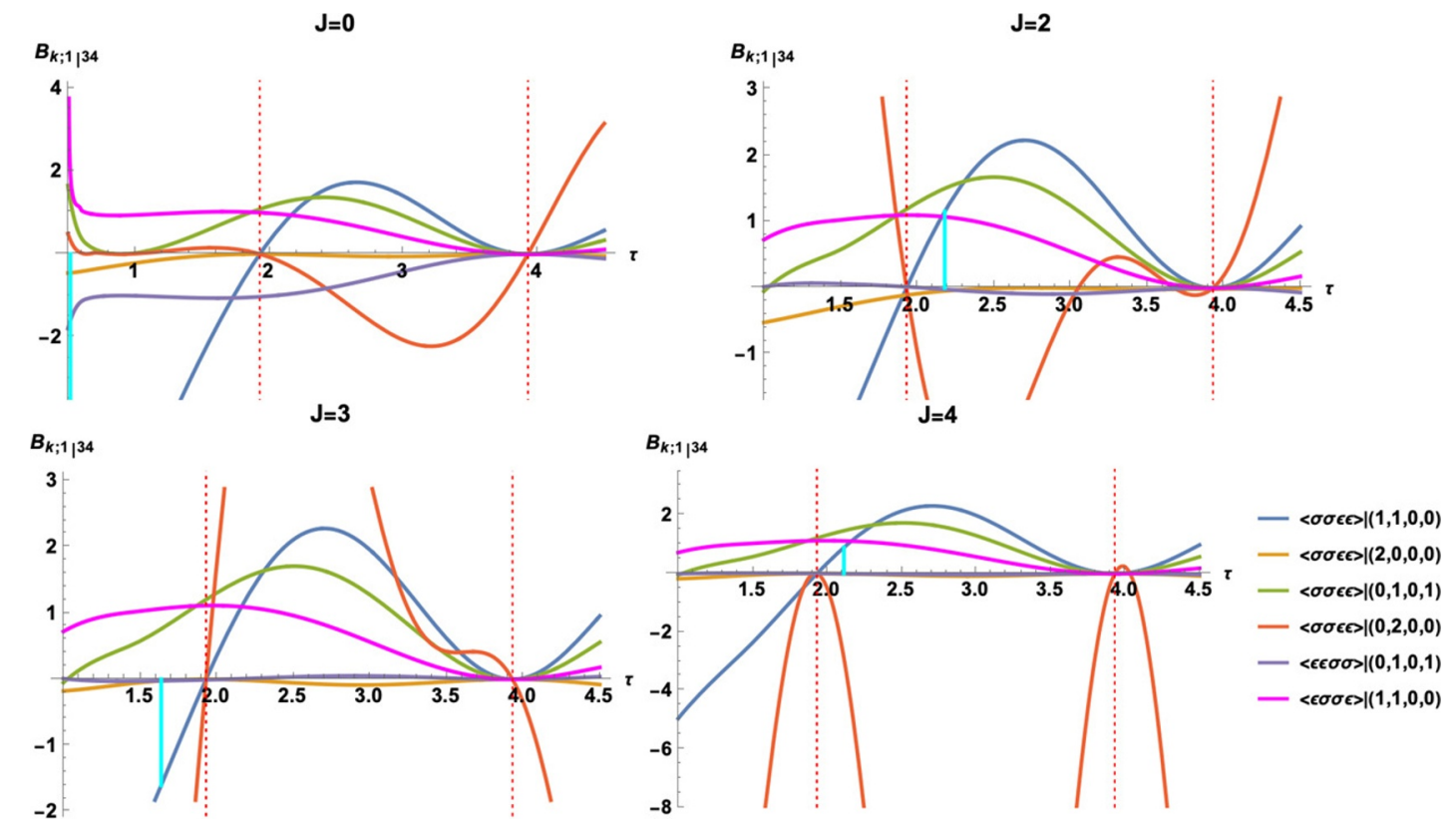

Figure 10. $B_{\mathbf{k} ; 1 \mid 34}$ for fixed spin $J$ as a function of twist $\tau$ for channels where odd $\mathbb{Z}_{2}$ operators are exchanged. We have included all three correlators $\langle\sigma \sigma \epsilon \epsilon\rangle,\langle\epsilon \epsilon \sigma \sigma\rangle$ and $\langle\epsilon \sigma \sigma \epsilon\rangle$ to highlight differences between subtraction schemes and operator ordering.
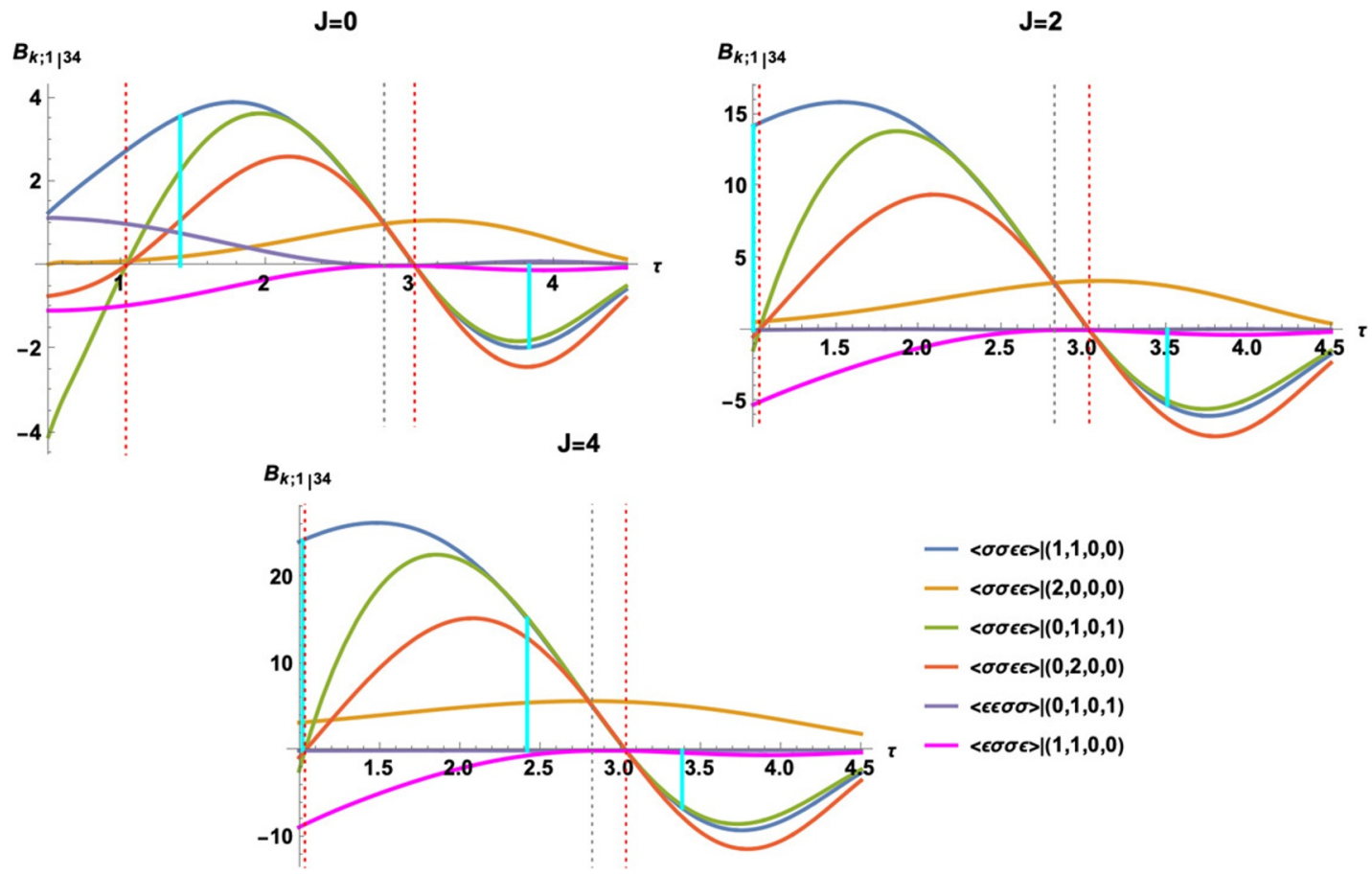

Figure 11. $B_{\mathbf{k} ; 1 \mid 34}$ for fixed spin $J$ as a function of twist $\tau$ for channels where even $\mathbb{Z}_{2}$ operators are exchanged. We have included all three correlators $\langle\sigma \sigma \epsilon \epsilon\rangle,\langle\epsilon \epsilon \sigma \sigma\rangle$ and $\langle\epsilon \sigma \sigma \epsilon\rangle$ to highlight differences between subtraction schemes and operator ordering. 


\begin{tabular}{|c|c|c|c|c|c|}
\hline $\mathcal{O}$ & $\mathbb{Z}_{2}$ & $J$ & $\tau_{\mathcal{O}}$ & $B_{(0,1,0,1) ; v \mid 34}$ & $B_{(0,1,0,1) ; v \mid 34}^{\prime}$ \\
\hline$\sigma$ & - & 0 & 0.518149 & 1 & 1 \\
\hline 1 & + & 0 & 0 & -0.473 & -0.628 \\
\hline$\epsilon$ & + & 0 & 1.41263 & -0.552 & -0.261 \\
\hline \multirow{2}{*}{\multicolumn{4}{|c|}{$\begin{array}{l}\text { Truncated sum } \\
\text { all operators } B^{s}+B^{t}\end{array}$}} & -0.025 & 0.111 \\
\hline & & & & -0.0002 & -0.102 \\
\hline
\end{tabular}

Table 11. Contribution of each operator to the $\langle\epsilon \epsilon \sigma \sigma\rangle$ sum rule with $v=1$; these results include the contribution of the OPE coefficients $f_{O_{i} O_{j} \mathcal{O}} f_{O_{l} O_{k} \mathcal{O}}$. The values are presented as ratios of the individual operator relative to the identity contribution. Empty cells contribute at order $O\left(10^{-2}\right)$ and less.

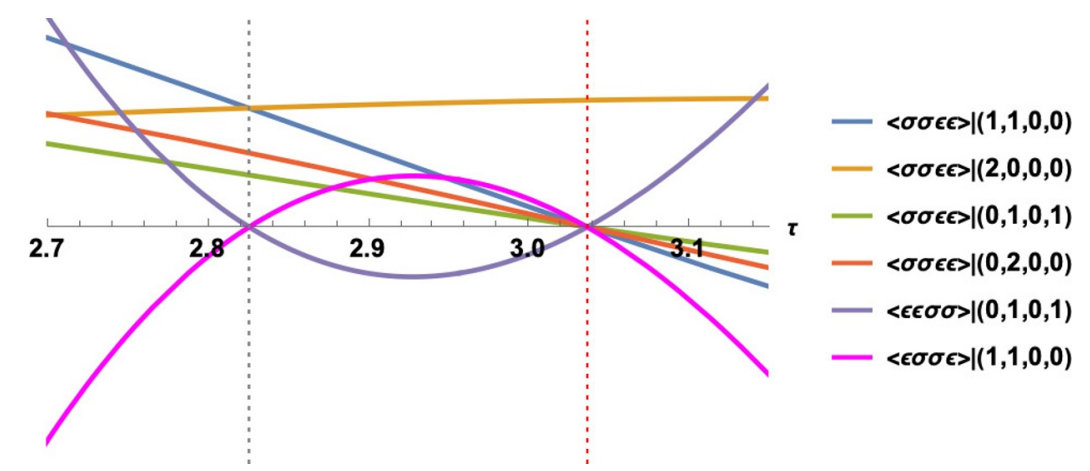

Figure 12. Close-up of the action of $B_{\mathbf{k} ; 1 \mid 34}$ functionals for even $\mathbb{Z}_{2}$ operators at $J=0$ near the leading $2 \Delta_{\epsilon}$ (grey vertical dotted line) and subleading $2 \Delta_{\sigma}+2$ (red vertical dotted line) double-twist values.

trajectory as shown by figure 12. Therefore, for $\tau<2 \Delta_{\epsilon}+2$, the sign of the functional is fixed by the number of $k_{12}$ subtractions. In particular, only the $(2,0,0,0)$ subtraction scheme yields a sign definite functional acting on $\tau<2 \Delta_{\epsilon}+2$. In the $t$-channel, sign definiteness is guaranteed for the $[\sigma \epsilon]_{0, J}$ family by including at least one $t$-channel subtraction. Otherwise, there is a sign change due to a simple zero at $\tau=\Delta_{\epsilon}+\Delta_{\sigma}$ as shown by the orange curve in figure 10. Therefore, we conclude that regardless of the subtraction scheme, at least one of the $s$ - or $t$-channel functional will be sign-definite for most operators: at large enough twist, the functionals become sign-definite except between the two double-twist families $[\epsilon \epsilon]_{n, J}$ and $[\sigma \sigma]_{n+1, J}$.

The zero structure sheds light into the contribution of various operators to the sum rule: by enhancing double-twist families with poles from $P_{\mathbf{k}}$, we further enhance contributions from all operators exchanged in that channel. For instances, as shown in table $9, s$-channel subtractions mostly balances a single odd $\mathbb{Z}_{2}$ operator against a small number of even $\mathbb{Z}_{2}$ operators. While we cannot predict which operator will dominate the sum rule, we observe empirically that the largest contributor to the sum rule is given by a low twist operator appearing in the channel where fewer subtractions are applied.

Let us briefly comment on the presence or absence of the identity in table 9. The identity saturates the lower bound in the Mellin-mandelstam space t as defined by eq. (4.10), and therefore, we expect the pole associated with the identity exchange to be located at 
the boundary $\mathrm{t}=\Delta+\Delta_{A}-\Delta_{B}-2 k_{34}$. Such a singularity in the Mellin-Mandelstam t-plane only appears if we include $t$-channel subtractions which generates poles of the form $\left(\Delta+\Delta_{A}-\Delta_{B}-2 k_{34}-\mathrm{t}\right)$ to cancel double-zeros from the Mack polynomial. Therefore, can cancel the double-zero by adding double poles with two $t$-channel subtractions $k_{23}$ and $k_{14}$ since the two trajectories are degenerate. By doing so, we can enhance the integrand with a double-poles which yields a residue of the form

$$
\frac{\Gamma(\Delta)}{\Gamma(\Delta / 2)^{2}} f\left(\Delta_{A}, \Delta_{B}\right) v^{-\Delta_{B}} \psi^{(0)}(\Delta / 2) .
$$

The above is non-zero as $\Delta \rightarrow 0$ due to the $\psi^{(0)}(\Delta / 2)$ factor. Thus, a non-vanishing $s$-channel identity only appears if we include symmetric subtractions along the $\tau_{23}$ and $\tau_{14}$ trajectories to enhance the Mellin integrand with a double-pole such that the identity contribution is

$$
\widehat{B}_{(0101) ; v \mid 34}^{s}\left[G_{0,0}^{s}\right]=-\Delta_{B} v^{-\Delta_{B}} \frac{\Gamma\left(\Delta_{A}-\Delta_{B}\right) \Gamma\left(\Delta_{B}\right)}{\Gamma\left(\Delta_{A}\right)} .
$$

This calculation also illustrates how the $\langle\sigma \sigma \epsilon \epsilon\rangle$ correlator differs from the $\langle\epsilon \epsilon \sigma \sigma\rangle$ one; while not apparent from figure 10 and 11 due to the scale, the asymmetric dependence on $\Delta_{B}$ suppresses the overall behaviour of $\widehat{B}_{\mathbf{k} ; v \mid 34}$ for the $\langle\epsilon \epsilon \sigma \sigma\rangle$ correlator. This non-linear suppression leads to better truncated sum rules as shown in table 11.

Finally, we note that the values in table 11 are similar to those in table 8 and therefore, we do not consider them linearly independent sum rules. This resemblance extends to apparent miror images between the $\langle\epsilon \epsilon \sigma \sigma\rangle$ and $\langle\epsilon \sigma \sigma \epsilon\rangle$ correlators for the spin $J=0$ curves in figure 10-11. It is unclear why these two correlators and their repsective subtraction schemes yield such similar results despite possessing clearly distinct Mellin-space integrands. Nevertheless, this emphasize that these mixed correlators can encode similar information through different operator ordering and subtraction schemes.

\subsection{Approximate solutions to crossing from truncated sum rules}

The previous section highlights the value of these dispersive functionals by suppressing the double-twist sectors thereby restricting the sum rule to a small number of low twist operators. Using these partial sum rules, this section aims to derive approximate solutions to crossing.

Deriving approximate solutions to crossing is an important step towards bounding CFT data using numerical minimization methods. Consider a linear combination of functionals denoted by $\mathcal{A}$ which approximately satisfy crossing for a finite number of exchanged operators:

$$
\mathcal{A}=\sum_{i=1}^{n} a_{i} B_{\mathbf{k} ; v \mid 34}^{s, t}\left[\mathcal{O}_{i}\right]=0,
$$

for coefficients $a_{i}$. By adding functionals that act positively on heavy operators and negatively for a small finite number of light operators, one could use optimization methods to bound CFT data. We take a first step towards tackling this optimization problem when using dispersive functionals by first evaluating $\mathcal{A}$ using insight from the previous subsection. 
As shown in the first half of table 4, there are 7 linearly independent sum rules that are restricted to three exchanged operators: $\sigma, \epsilon$ and $T_{\mu \nu}$. These sum rules follow from three correlators:

$$
\begin{array}{rlrl}
\langle\sigma \sigma \sigma \sigma\rangle: & \mathbf{1}-\mathbf{1}_{\mathrm{u}}+f_{\sigma \sigma \epsilon}^{2} B[\epsilon]+f_{\sigma \sigma T}^{2} B\left[T_{\mu \nu}\right] & =0 \\
f_{\sigma \sigma \epsilon}^{2} \Phi_{2}[\epsilon]+f_{\sigma \sigma T}^{2} \Phi_{2}\left[T_{\mu \nu}\right] & =0 \\
\mathbf{1}+f_{\sigma \sigma \epsilon} f_{\epsilon \epsilon \epsilon} B[\epsilon]+f_{\sigma \sigma \epsilon}^{2} B[\sigma] & =0 \\
\langle\epsilon \sigma \sigma \epsilon\rangle: & f_{\sigma \sigma \epsilon}^{2} B[\sigma]+f_{\sigma \sigma \epsilon} f_{\epsilon \epsilon \epsilon} B[\epsilon]+f_{\sigma \sigma T} f_{\epsilon \epsilon T} B\left[T_{\mu \nu}\right] & =0
\end{array}
$$

There are therefore 5 unknowns in this system of equations:

$$
\Delta_{\sigma}, \quad \Delta_{\epsilon}, \quad, f_{\sigma \sigma \epsilon}, \quad f_{\epsilon \epsilon \epsilon}, \quad \sqrt{c}=\frac{\sqrt{\Delta_{i} \Delta_{j}}}{f_{i j T}} .
$$

Using our sum rules, we can find approximate solutions to crossing for 3D Ising model operators by minimizing the stress-tensor coupling according to a $c$-minimization procedure [6].

To implement this procedure, we cancel the $f_{\epsilon \epsilon \epsilon}$ coupling by taking linear combination of the $B_{\mathbf{k} ; 1 \mid m n}$ and its derivative. Unlike the equal operator sum rule, the mixed correlator ones are not sign definite, and therefore we allow for the sum rule to be satisfied up to $\pm 10^{-1}$ error given the errors tabulated in the previous section. Using the notation $B_{\left\langle\phi_{1} \phi_{2} \phi_{3} \phi_{4}\right\rangle}[\mathcal{O}]$ to denote the action of the $B_{(1,1,0,0) ; 1 \mid 34}^{s, t}$ collinear functional with fixed operator ordering and exchange operator $\mathcal{O}$, we then construct the following inequalities:

$$
\begin{aligned}
0 \leq & \left.B_{\sigma \sigma \sigma \sigma} \mathbf{1}\right]-\mathbf{1}_{\mathrm{u}}+f_{\sigma \sigma \epsilon}^{2} B_{\sigma \sigma \sigma \sigma}[\epsilon]+f_{\sigma \sigma T}^{2} B_{\sigma \sigma \sigma \sigma}\left[T_{\mu \nu}\right] \\
0 \leq & B_{\sigma \sigma \sigma \sigma}^{\prime}[\mathbf{1}]-\mathbf{1}_{\mathrm{u}}+f_{\sigma \sigma \epsilon}^{2} B_{\sigma \sigma \sigma \sigma}^{\prime}[\epsilon]+f_{\sigma \sigma T}^{2} B_{\sigma \sigma \sigma \sigma}^{\prime}\left[T_{\mu \nu}\right] \\
0 \leq & f_{\sigma \sigma \epsilon}^{2} \Phi_{2}[\epsilon]+f_{\sigma \sigma T}^{2} \Phi_{2}\left[T_{\mu \nu}\right] \\
-0.1 \leq & \left(B_{\epsilon \sigma \sigma \epsilon}[\mathbf{1}]-\frac{B_{\epsilon \sigma \sigma \epsilon}[\epsilon]}{B_{\epsilon \sigma \sigma \epsilon}^{\prime}[\epsilon]} B_{\epsilon \sigma \sigma \epsilon}^{\prime}[\mathbf{1}]\right) \\
& +f_{\sigma \sigma \epsilon}^{2}\left(B_{\epsilon \sigma \sigma \epsilon}[\sigma]-\frac{B_{\epsilon \sigma \sigma \epsilon}[\epsilon]}{B_{\epsilon \sigma \sigma \epsilon}^{\prime}[\epsilon]} B_{\epsilon \sigma \sigma \epsilon}^{\prime}[\sigma]\right) \leq 0.1 \\
-0.1 \leq & f_{\sigma \sigma \epsilon}^{2}\left(B_{\sigma \sigma \epsilon \epsilon}[\sigma]-\frac{B_{\sigma \sigma \epsilon \epsilon}[\epsilon]}{B_{\sigma \sigma \epsilon \epsilon}^{\prime}[\epsilon]} B_{\sigma \sigma \epsilon \epsilon}^{\prime}[\sigma]\right) \\
& +f_{\sigma \sigma T} f_{\epsilon \epsilon T}\left(B_{\sigma \sigma \epsilon \epsilon}\left[T_{\mu \nu}\right]-\frac{B_{\sigma \sigma \epsilon \epsilon}[\epsilon]}{B_{\sigma \sigma \epsilon \epsilon}^{\prime}[\epsilon]} B_{\sigma \sigma \epsilon \epsilon}^{\prime}\left[T_{\mu \nu}\right]\right) \leq 0.1
\end{aligned}
$$

Since the functional $B_{\mathbf{k} ; v \mid 34}^{s, t}$ is a non-linear function of $\Delta_{\sigma}, \Delta_{\epsilon}$, we sample points within a grid $\left(\Delta_{\sigma}, \Delta_{\epsilon}\right) \in([0.5,0.66],[1.34,1.48])$ and interpolate its action within that domain. By minimizing the stress-tensor coupling $c$ using the NMinimize function in Mathematica, the best approximation to the Ising model data is recorded in tab 12 for the four remaining unknowns in our system of equations. We underscore the necessity to use all five inequalities to obtain reasonable crossing solutions despite the fact that the system of equations only includes 4 unknowns.

Despite the small grid size, our results are comparable to those in [5]. We observed empirically that better accuracy can be obtained by increasing the number of data points 


\begin{tabular}{|c|cccc|}
\hline & $\Delta_{\sigma}$ & $\Delta_{\epsilon}$ & $f_{\sigma \sigma \epsilon}$ & $c$ \\
\hline Results from [5] & 0.518149 & 1.41263 & 1.05185 & 2.5241 \\
Results from truncated dispersive sum rules & 0.512390 & 1.42934 & 1.01279 & 2.6370 \\
\hline
\end{tabular}

Table 12. 3D Ising model data derived from 7 truncated dispersive sum rules that combine to provide 5 inequalities. These results were obtained by sampling $N_{\Delta_{\sigma}} \times N_{\Delta_{\epsilon}}=42 \times 40$ points distributed quadratically around their expected values.

within the grid. One could also perform a two-dimensional quadratic (or higher order) fit of the $B_{\mathbf{k} ; v \mid 34}$ functionals within a domain near the expect Ising model values, however the mixed correlator functionals diverge near the unitarity bound thereby forcing one to increase the size of the dataset to obtain high precision fits; we found the use of interpolation functions to be better suited (although less rigorous) for smaller datasets. This exercise simply served to illustrate the effectiveness of these dispersive functionals. We expect higher precision is achievable by increasing the number of sampling data points, by increasing the number of functionals and inequalities, or by using combinations of functionals with only single-sided inequalities.

\section{Discussion}

In this paper, we extend the study of dispersive CFT functionals to mixed correlators. We first conduct our analysis in position-space (section 3) where our main result is the derivation of the mixed correlator dispersive kernel given by eq. (3.18). Convergence, positivity and analyticity properties are examined in section 3.3 where we found that the first two depend on the subtraction scheme $\mathbf{k}=\left(k_{12}, k_{23}, k_{34}, k_{14}\right)$ and the operator ordering; we summarized the convergence conditions in table 2 . We conduct an analogous derivation of the functionals in Mellin-space (section 4) where the Mellin representation of the functional is given by eq. (4.8). The benefits of working in Mellin-space are threefold: the zero and pole structure is manifest, projection functionals similar to $\Phi_{\ell}$ are easier to construct, and they are easier to evaluate numerically. This comes at the cost of obscuring convergence and positivity properties that were clearer in position-space. Equipped with both the position-space and Mellin-space representation of the dispersive collinear functional $B_{\mathbf{k} ; v \mid m n}$, we extended the work of [31] by constructing mixed correlator holographic functionals $C_{\mathbf{k} ; \nu \mid m n}$ in sections 3.4 and 4.2 in position- and Mellin-space respectively.

Our sum rules follow from eq. (2.12) such that for large enough twist, each $s$ - and $t$ channel functional become sign definite. Therefore, cancellations occur between low twist operators which allows one to construct truncated sum rules. We verify this statement in section 5.1 by studying spin-2 convergent non-log sum rules applied to five 3D Ising correlators. As mentioned previously, our dispersive sum rules are not invariant under operator ordering due to expanding in the collinear limit, and therefore we conduct an extensive analysis for sum rules with different subtraction schemes and operator ordering to find those dominated by the fewest lowest twist operators. Our results indicate that crossing-symmetric subtraction schemes are the most effective in that regard since they 
enhance the fewest operators in each channel. One may build other functionals similar to those in $[32,33]$ to isolate other operators in the sum rule rather than the lowest twist ones. Asymmetric subtraction schemes also appear to be useful for correlators of heavy external operators as indicative by results for the $\langle\epsilon \epsilon \epsilon \epsilon\rangle$ correlator in section 5.1.2.

In section 5.2, we aimed to derive approximate solutions to crossing by using a set of truncated dispersive sum rules. Using only 5 inequalities, two of which follow from mixed correlators, we were able to obtain reasonable approximations to the 3D Ising model as shown in table 12 by evaluating the functionals within a grid of $\left(\Delta_{\sigma}, \Delta_{\epsilon}\right)$, and interpolating its action before minimizing the stress-tensor coupling.

While our method appears promising, errors are difficult to constrain due to the lack of sign definiteness for mixed correlators sum rules, and our methodology requires sampling a large number of data points to find high precision minima. A much more effective method is to leverage positivity properties of these functionals by constructing a semidefinite matrix amenable to the use of semidefinite programming. Following [7] (see eq. (3.11) and (3.12) of that paper), a fixed-u convention positive semi-definite matrix can be constructed as follows:

$$
\left(\begin{array}{ll}
1 & 1
\end{array}\right) \vec{\alpha} \cdot \vec{V}_{+, 0,0}\left(\begin{array}{l}
1 \\
1
\end{array}\right)+\sum_{\mathcal{O} \in \mathbb{Z}_{2}^{+}}\left(f_{\sigma \sigma \mathcal{O}} f_{\epsilon \in \mathcal{O}}\right) \vec{\alpha} \cdot \vec{V}_{+, \Delta, J}\left(\begin{array}{c}
f_{\sigma \sigma \mathcal{O}} \\
f_{\epsilon \epsilon \mathcal{O}}
\end{array}\right)+\sum_{\mathcal{O} \in \mathbb{Z}_{2}^{-}} f_{\sigma \epsilon \mathcal{O}}^{2} \vec{\alpha} \cdot \vec{V}_{-, \Delta, J}=0
$$

where

$$
\begin{array}{ll}
\vec{\alpha} \cdot \vec{V}_{+, \Delta, J} \succeq 0 & \forall \mathbb{Z}_{2}^{+} \text {operators with even spin, } \\
\vec{\alpha} \cdot \vec{V}_{-, \Delta, J} \geq 0 & \forall \mathbb{Z}_{2}^{-} \text {operators in the spectrum. }
\end{array}
$$

For some dispersive functional $W^{s, t}$, whether it be the $B_{\mathbf{k}: v \mid 34}^{s, t}$ functional presented in this paper or new projected dispersive functionals, the matrix elements of $V_{ \pm, \Delta, J}$ would include linear combinations of s- and t-channel functionals:

$$
F_{ \pm, \Delta, J}^{i j, k l}=W^{s}\left[G_{\Delta, J}^{\Delta_{i j}, \Delta_{k l}}(u, v)\right] \pm W^{t}\left[G_{\Delta, J}^{\Delta_{k j}, \Delta_{i l}}(u, v)\right] .
$$

It would be interesting to study this mixed correlator functional matrix more extensively. Moreover, it would be interesting to test whether navigator functions [47, 48] built from dispersive functionals may be more effective.

Beyond their numerical implementations, there are other avenues to explore. Consider the $\Phi_{2}$ functional for example. Convergence properties of this functional should be further studied in light of our results for the $\langle\epsilon \epsilon \epsilon \epsilon\rangle$ correlator in section 5.1.2. Furthermore, the numerical evaluation becomes expensive for large spin operators given the oscillatory nature of the Mack polynomials. A position-space version of these functionals could prove more scalable since conformal blocks are better behaved at large spin. One could further explore other types of projection operators for mixed correlators building upon the early analysis conducted in appendix D.

Finally, the derivation of mixed correlator holographic functionals in sections 3 and 4.2 provides a new pathway to probe certain features of AdS quantum gravity. In particular, these functionals could serve helpful to study heavy-heavy-light-light CFT correlators which are interpreted as a probe particle travelling in a black hole geometry [49-51]. Furthermore, 
the simplicity in eq. (3.57) and (4.21) encourages the construction of spinning functionals that would allow one to bootstrap gravitons in AdS which may further shed light on the relations between flat-space and AdS scattering.

\section{Acknowledgments}

I thank Frank Coronado, Clément Virally and Zahra Zahraee for discussions throughout this project. I am especially thankful for the discussions and the support from Simon CaronHuot. The work of AT is supported by the National Science and Engineering Council of Canada and the Simons Collaboration on the Nonperturbative Bootstrap.

\section{A Detailed derivation of the $\mathfrak{B}_{\mathfrak{k} \mid m n}^{\mathfrak{a}, \mathfrak{b}}$ kernel}

To be explicit, we derive the $\mathfrak{B}_{\mathbf{k} \mid 34}^{\mathfrak{a}, \mathfrak{b}}$ kernel by starting with eq. (3.17) which is a triple integral in the Mellin mandelstam variables $\mathrm{s}^{\prime}, \mathrm{t}^{\prime}$ and $\mathrm{s}$. We first deform the s contour to evaluate the residue at $s=\Delta_{3}+\Delta_{4}+2 k_{34}$, followed by explicitly resuming the $\mathrm{t}^{\prime}$ residues at $\Delta_{2}+\Delta_{3}+2 k_{23}+2 m$ and $\Delta_{1}+\Delta_{4}+2 k_{14}+2 m$ by deforming the contour to the right. Finally, the $s^{\prime}$ contour is deformed to the left to pick up the poles at $\Delta_{1}+\Delta_{2}+k_{12}-2 m$. Let us consider explicitly the resumed poles at $\mathrm{t}^{\prime}=\Delta_{2}+\Delta_{3}+2 k_{23}+2 m$ :

$$
\begin{aligned}
& \left.\mathfrak{B}_{\mathbf{k} \mid 34}^{\mathfrak{a}, \mathfrak{b}}\right|_{\mathbf{t}^{\prime}=\Delta_{2}+\Delta_{3}+2 k_{23}+2 m}=\sum_{m_{s}=0}^{\infty} \frac{\left(u^{\prime}\right)^{m_{s}-k_{12}}\left(v^{\prime}\right)^{p+q+k_{12}-k_{23}-k_{34}-m_{s}-2} v^{k_{23}} u^{-p-q+k_{34}}}{2 \pi \Gamma\left(p+q+k_{12}-k_{34}-m_{s}-1\right) \Gamma\left(m_{s}+1\right)} \\
& \times(-1)^{m_{s}-k_{12}} \frac{\Gamma\left(p-q+k_{14}-k_{23}\right) \Gamma\left(p+q+k_{12}-k_{34}\right) \csc \left(\pi\left(p+q+k_{12}-m_{s}-1\right)\right)}{\left(p+q+k_{12}-k_{34}-m_{s}-1\right) \Gamma\left(-p-q-k_{12}+k_{34}+m_{s}+1\right)} \\
& \times \frac{{ }_{2} F_{1}\left(-p-q-k_{12}+k_{34}+m_{s}+2,-2 p-k_{12}-k_{14}+k_{23}+k_{34}+m_{s}+2 ;-p+q-k_{14}+k_{23}+1 ; \frac{v}{v^{\prime}}\right)}{\Gamma\left(2 p+k_{12}+k_{14}-k_{23}-k_{34}-m_{s}-1\right)},
\end{aligned}
$$

where $m_{s}$ labels the poles originating from the $\mathrm{s}^{\prime}$ contour deformation, and

$$
p=\frac{\Delta_{13}}{2}, \quad q=\frac{\Delta_{24}}{2} .
$$

Unfortunately, this sum cannot be performed straightforwardly. To progress, we write an ansatz of a hypergeometric as a function of $\chi$ as defined by eq. (3.19) and we match its arguments term-by-term by expanding around $u^{\prime}, v \rightarrow 0$. By doing so, we find

$$
\begin{aligned}
& \left.\mathfrak{B}_{\mathbf{k} \mid 34}^{\mathfrak{a}, \mathfrak{b}}\right|_{\mathrm{t}^{\prime}=\Delta_{2}+\Delta_{3}+2 k_{23}+2 m}=\frac{(-1)^{1-k_{12}} 2^{2 p+k_{12}+k_{14}-k_{23}-k_{34}-4}}{\pi^{2} \Gamma\left(2 p+k_{12}+k_{14}-k_{23}-k_{34}-1\right)}\left(-u^{\prime}+v^{\prime}+v\right) \\
& \quad \times v^{\frac{1}{2}\left(2 p+k_{12}+k_{14}+k_{23}-k_{34}-3\right)} \chi^{\frac{1}{2}\left(-2 p-k_{12}-k_{14}+k_{23}+k_{34}+3\right)} u^{-p-q+k_{34}}\left(u^{\prime}\right)^{-k_{12}} \\
& \quad \times \csc \left(\pi\left(p+q+k_{12}\right)\right)\left(v^{\prime}\right)^{\frac{1}{2}\left(2 q+k_{12}-k_{14}-k_{23}-k_{34}-3\right)} \sin \left(\pi\left(p+q+k_{12}-k_{34}\right)\right) \\
& \quad \times \Gamma\left(p-q+k_{14}-k_{23}\right) \Gamma\left(p+q+k_{12}-k_{34}\right) \\
& \quad \times{ }_{2} F_{1}\left(\frac{2 q+k_{12}-k_{14}+k_{23}-k_{34}}{2}, \frac{-2 p-k_{12}-k_{14}+k_{23}+k_{34}+3}{2} ;-p+q-k_{14}+k_{23}+1 ;-\chi\right) .
\end{aligned}
$$


We can now combine the two $t^{\prime}$ towers of poles by using the following identity:

$$
\begin{aligned}
{ }_{2} F_{1}(a, b, c, z)= & \frac{(-z)^{-a}(\Gamma(c) \Gamma(b-a))}{\Gamma(b) \Gamma(c-a)}{ }_{2} F_{1}\left(a, a-c+1 ; a-b+1 ; \frac{1}{z}\right) \\
& +\frac{(-z)^{-b}(\Gamma(c) \Gamma(a-b))}{\Gamma(a) \Gamma(c-b)}{ }_{2} F_{1}\left(b-c+1, b ;-a+b+1 ; \frac{1}{z}\right),
\end{aligned}
$$

which allows us to obtain our final quoted result in eq. (3.18).

To derive the full dispersion kernel for mixed correlators, one would have to resum poles originating from the s-contour. A well-posed ansatz to resum these poles would be a hypergeometric function with the ratio defined on the l.h.s. of eq. (3.19) as an argument. One could fix the $p, q$ dependence by matching the series expansion.

\section{B $\quad \mathfrak{B}_{\mathrm{k} \mid m n}^{\mathfrak{a}, \mathfrak{b}}$ kernel simplification}

We explain simplicity of the equal operator kernel found in [30]. For concreteness, let us focus on the $\mathfrak{B}_{\mathbf{k} \mid 34}^{\mathfrak{a}, \mathfrak{b}}\left(u^{\prime}, v^{\prime}\right)$ kernel which contains the following hypergeometric function:

$$
\mathfrak{B}_{\mathbf{k} \mid 34}^{\mathfrak{a}, \mathfrak{b}} \supset{ }_{2} F_{1}\left(\frac{k_{12}+k_{14}-k_{23}+\Delta_{13}}{2}, \frac{k_{12}-k_{14}+k_{23}+\Delta_{24}}{2} ; k_{12}+\frac{\Delta_{13}+\Delta_{24}}{2}-\frac{1}{2} ;-\frac{1}{\chi}\right) .
$$

Without loss of generality, we set $k_{34}=0$ in the above. Since hypergeometric functions ${ }_{2} F_{1}$ are symmetric in its first two arguments, we see that subtractions in $k_{23}$ and $k_{14}$ are antisymmetric relative to a fixed point determined by the u-channel double-twist values $\frac{\Delta_{13}}{2}$ and $\frac{\Delta_{24}}{2}$.

For pairwise external operators, this hypergeometric function simplifies for the three cases where double-twist operators can be exchanged in the $s$-channel; this corresponds to the cases where $\Delta_{13}=-\Delta_{24}$ or $\Delta_{13}=\Delta_{24}=0$. In such cases, the third argument of the hypergeomtric function reduces to $k_{12}-\frac{1}{2}$. When $k_{12}=1$, the hypergeomtric function further reduces to

$$
\left.\mathfrak{B}_{\left(1, k_{23}, 0, k_{14}\right) \mid 34}^{\mathfrak{a}, \mathfrak{b}}\right|_{\Delta_{13}=-\Delta_{24}} \supset{ }_{2} F_{1}\left(\frac{1}{2}+y, \frac{1}{2}-y, \frac{1}{2},-\frac{1}{\chi}\right)=\frac{\left(\frac{\sqrt{\chi+1}-1}{\sqrt{\chi}}\right)^{y}+\left(\frac{\sqrt{\chi+1}-1}{\sqrt{\chi}}\right)^{-y}}{2 \sqrt{\frac{1}{\chi}+1}} .
$$

where $y=k_{14}-k_{23}+\Delta_{13}$. This explains why the equal operator kernel in [30] was simpler: a single subtraction along the $\tau_{12}$ trajectory allows for this reduction.

For the $\langle A A B B\rangle$ where $\Delta_{13}=\Delta_{24}$, there is no possible (generic) simplification since $\Delta_{13} \in \mathbb{R}$ and the $\Delta_{13}$ dependence in the third argument of the hypergeometric function prevents any further simplifications.

\section{Mack polynomials}

We write explicit formulae for Mack polynomials with unequal external operators which play a key role in describing Mellin amplitudes $M(\mathrm{~s}, \mathrm{t})$. Mack Polynomials are well- 
documented [38, 41, 42, 52]. We hope to provide a more compact notation that may hopefully help readers. ${ }^{11}$

Mellin amplitudes have poles when the Mellin mandelstam variable s approaches the twist of exchanged operators $\mathcal{O}$ :

$$
M(\mathrm{~s}, \mathrm{t}) \propto M^{s}(\mathrm{~s}, \mathrm{t}) \sim \frac{\mathcal{Q}_{\Delta_{\mathcal{O}}, J_{\mathcal{O}}}^{m, \Delta_{i}}(\mathrm{t})}{\mathrm{s}-\tau_{\mathcal{O}}-2 m}
$$

where the kinematical polynomial $\mathcal{Q}_{\Delta, J}^{m, \Delta_{i}}$ takes the form

$$
\mathcal{Q}_{\Delta, J}^{m, \Delta_{i}}(\mathrm{t})=k_{\Delta, J}^{m, \Delta_{i}} Q_{\Delta, J}^{m, a, b}(\mathrm{t})
$$

where $a, b$ is given by eq. (3.4), and

$$
\begin{aligned}
k_{\Delta, J}^{m, \Delta_{i}}= & \frac{1}{\Gamma\left(\frac{1}{2}\left(J+\Delta+\Delta_{1}-\Delta_{2}\right)\right) \Gamma\left(\frac{1}{2}\left(J+\Delta-\Delta_{1}+\Delta_{2}\right)\right) \Gamma\left(\frac{1}{2}\left(J-\Delta+\Delta_{1}+\Delta_{2}\right)-m\right)} \\
& \times \frac{1}{\Gamma\left(\frac{1}{2}\left(J+\Delta-\Delta_{3}+\Delta_{4}\right)\right) \Gamma\left(\frac{1}{2}\left(J+\Delta+\Delta_{3}-\Delta_{4}\right)\right) \Gamma\left(\frac{1}{2}\left(J-\Delta+\Delta_{3}+\Delta_{4}\right)-m\right)} \\
& \times \frac{2 \Gamma(J+\Delta-1) \Gamma(J+\Delta)}{\Gamma(\Delta-1) \Gamma(m+1)\left(-\frac{d}{2}+\Delta+1\right)_{m}} .
\end{aligned}
$$

$Q_{\Delta, J}^{m, a, b}(\mathrm{t})$ is the so-called Mack polynomial.

Below, we reorganize results from [41, 52] by first generalizing the Mack polynomials from [41], and then verifying against the appendices in [52]. The sums in [41] were then re-organized in order to isolate the $J$ dependence at low $m$ for $Q_{\Delta, J}^{0, a, b}$. The recursion relation reads

$$
Q_{\Delta, J}^{m, a, b}(t)=\sum_{q=0}^{\min (m, J)} \sum_{p=0}^{q} \mathfrak{q}_{\Delta, J}^{a, b}(p, q)(-m)_{q} Q_{\Delta+p, J-q}^{0, a-\frac{q-p}{2}, b+\frac{q-p}{2}}(\mathrm{t})
$$

where

$$
\begin{aligned}
\mathfrak{q}_{\Delta, J ; d}^{a, b}(p, q)= & \frac{J !(-1)^{p+q}}{p !(J-q) !(q-p) !} \frac{\left(\frac{d-2}{2}+J\right)_{-q}(J+\Delta-1)_{p-q}}{\left(a+\frac{J+\Delta}{2}\right)_{p-q}\left(\frac{J+\Delta}{2}-b\right)_{p-q}} \\
& \times \frac{\left(\frac{1}{2}(-d-J+\Delta+2)+a\right)_{p}\left(\frac{1}{2}(-d-J+\Delta+2)-b\right)_{p}}{(-d-J+\Delta+2)_{p}}
\end{aligned}
$$

and the $m=0$ Mack polynomial is given by the following closed-form expression

$$
Q_{\Delta, J}^{0, a, b}(\mathrm{t})=\frac{\left(a+\frac{\Delta-J}{2}\right)_{J}\left(b+\frac{\Delta-J}{2}\right)_{J}}{(\Delta-1)_{J}}{ }_{3} F_{2}\left(-J,-\frac{\mathrm{t}}{2}, \Delta-1 ; a+\frac{\Delta}{2}-\frac{J}{2}, b+\frac{\Delta}{2}-\frac{J}{2} ; 1\right) .
$$

\footnotetext{
${ }^{11}$ We thank Simon Caron-Huot for experimentations that led to this formulae, and for sharing the Mathematica notebook.
} 


\section{Mixed correlator analytic double-twist functionals}

In [30], a projection functional $\Phi_{\ell}$ was introduced with the following properties:

1. It is positivie definite above a threshold $\tau^{*}$.

2. It returns double-zeros on all double-twists with spin $J \in 2 \mathbb{Z} \neq \ell$.

3. It returns a simple zero on the leading double-twist $n=0$ for $J=\ell \in 2 \mathbb{Z}$.

These properties are well suited for the numerical bootstrap. To study these projection operators, it is convenient to expand the $\widehat{B}_{\mathbf{k} ; t \mid m n}$ Mellin functionals using the basis of analytic double-twist functionals introduced earlier in eq. (4.18) which we rewrite here schematically ${ }^{12}$ in Mellin-space for convenience:

$$
M_{\mathbf{k}}^{s, t}(\mathrm{~s}, \mathrm{t})=\sum_{n, \ell} \alpha_{n, \ell}^{s, t}\left[M_{\mathbf{k}}\right] \widehat{a}_{n, \ell}^{\Delta_{i}, s, t}(\mathrm{~s}, \mathrm{t})+\beta_{n, \ell}^{s, t}\left[M_{\mathbf{k}}\right] \widehat{b}_{n, \ell}^{\Delta_{i}, s, t}(\mathrm{~s}, \mathrm{t}) .
$$

By evaluating the Mellin amplitude (correlator) in the $s$-channel collinear limit, we restrict ourselves to the leading double-twist $n=0$ sector. In general, for the collinear $\widehat{B}_{\mathbf{k} ; t \mid m n}$ functionals, we have for arbitrary subtraction schemes

$$
\widehat{B}_{\mathbf{k} ; \mathrm{t} \mid m n}^{s, t}=\sum_{\ell} \alpha_{0, \ell}^{s, t} \widehat{a}_{\ell \mid m n}^{\Delta_{i}, s, t}(\mathrm{t})+\beta_{0, \ell}^{s, t} \widehat{b}_{\ell \mid m n}^{\Delta_{i}, s, t}(\mathrm{t}),
$$

where the expansion coefficients were defined in eq. (4.18)we will drop the descendant label $n$ since $n=0$ for $\widehat{B}_{\mathbf{k} ; \mathbf{t} \mid m n}$ functionals. Some coefficients $\widehat{a}$ or $\widehat{b}$ may be absent depending on the correlator configuration and the subtraction scheme. For example, the $\langle A A B B\rangle$ correlator with $\mathbf{k}=(2,0,0,0)$ can be expanded in the following basis:

$$
\begin{aligned}
\widehat{B}_{(2,0,0,0) ; v \mid 34}^{t}= & O\left(\left(\Delta-\Delta_{A}-\Delta_{B}-J\right)^{2}\right), \\
\widehat{B}_{(2,0,0,0) ; v \mid 34}^{s}= & \sum_{\ell} \alpha_{\ell, 2 \Delta_{A}}^{s} \widehat{a}_{\ell \mid 34,2 \Delta_{A}}^{\Delta_{i}, s}(\mathrm{t})+\beta_{\ell, 2 \Delta_{A}}^{s} \widehat{b}_{\ell \mid 34,2 \Delta_{A}}^{\Delta_{i}, s}(\mathrm{t}) \\
& +\alpha_{\ell, 2 \Delta_{B}}^{s} \widehat{a}_{\ell \mid 34,2 \Delta_{B}}^{\Delta_{i}, s}(\mathrm{t})+\beta_{\ell, 2 \Delta_{B}}^{s} \widehat{b}_{\ell \mid 34,2 \Delta_{B}}^{\Delta_{i}, s}(\mathrm{t}),
\end{aligned}
$$

where we have added an additional subscript to $\widehat{a}, \widehat{b}$ to label the double-twist family. The presence of $\widehat{a}, \widehat{b}$ can be understood from the zero structure of Mack polynomials and subtraction schemes as discussed at the end of section 4.1. This foreshadows a prominent challenge in constructing a projection functional for mixed correlators: it is difficult to find a basis that diagonalizes the sum rule in both $s$ - and $t$-channels. This subsection is devoted to elaborating on this remark.

Before tackling the mixed correlator case, let us briefly sketch and review the derivation of the projection functional $\Phi_{\ell}$ for equal operators first introduced in [30]. Such a functional is obtained by integrating $\widehat{B}_{2, \mathrm{t}}$ against a kernel $f_{\ell}(\mathrm{t})$

$$
\Phi_{\ell}=\int_{\Delta_{\phi}-i \infty}^{\Delta_{\phi}+i \infty} \frac{d \mathrm{t}}{2 \pi i} f_{\ell}(\mathrm{t}) \widehat{B}_{2, \mathrm{t}}
$$

\footnotetext{
${ }^{12}$ For mixed correlators, there are additional labels to identify the expansion trajectory and the doubletwist family.
} 
where $f_{\ell}(\mathrm{t})$ can be shown to be

$$
f_{\ell}(\mathrm{t})=\frac{\left(2 \Delta_{\phi}+2 \ell-1\right) \Gamma\left(\Delta_{\phi}+\ell\right)^{4} \Gamma\left(2 \Delta_{\phi}+\ell-1\right)}{\Gamma(\ell+1) \Gamma\left(2 \Delta_{\phi}+2 \ell\right)^{2}} \int_{\Delta_{\phi}}^{\mathrm{t}} d x \Gamma_{\Delta_{\phi}}^{4}(x) a_{\ell}(x) .
$$

$\Phi_{\ell}$ is evaluated by using integration by parts. The second property at the top of this subsection can be satisfied by leveraging the orthogonality relations between Mack polynomials. The third property is satisfied if $f_{\ell}(\mathrm{t})$ is an odd function with respect to $\mathrm{t}$ :

$$
f_{\ell}(\mathrm{t})=-f_{\ell}\left(2 \Delta_{\phi}-\mathrm{t}\right) .
$$

Together, properties 2 and 3 suggest that $f_{\ell}(\mathrm{t}) \propto \widehat{b}_{\ell}(\mathrm{t})$. To obtain a concise closed-form expression for $f_{\ell}(\mathrm{t})$, one can relate the linear double-twist coefficient $\widehat{b}_{\ell}(\mathrm{t})$ to the zeroth order coefficient $\widehat{a}_{\ell}(\mathrm{t})$ as follows:

$$
\widehat{b}_{\ell}(\mathrm{t})-\widehat{b}_{\ell}\left(2 \Delta_{\phi}-\mathrm{t}\right)=-\frac{d \widehat{a}_{\ell}(\mathrm{t})}{d \mathrm{t}},
$$

thereby allowing us to use orthogonality properties of the Mack polynomial. To better understand the importance of this symmetry, let us consider the equal operator case such that we lose the double-twist family subscript $(m n)$. One can show that for the spin-2 subtracted $\widehat{B}_{2 ; \mathrm{t}}$ equal operator functional, the individual $s$ - and $t$-channel Mack polynomial allow for the following expansion around double-twist values:

$$
\begin{aligned}
& \left.\widehat{B}_{2 ; \mathrm{t}}^{s}\right|_{\Delta=2 \Delta_{\phi}+\ell}=\widehat{a}_{\ell}^{s}(\mathrm{t})+\widehat{b}_{\ell}^{s}(\mathrm{t})\left(\Delta-2 \Delta_{\phi}-\ell\right)+O\left(\left(\Delta-2 \Delta_{\phi}-\ell\right)^{2}\right), \\
& \left.\widehat{B}_{2 ; \mathrm{t}}^{t}\right|_{\Delta=2 \Delta_{\phi}+\ell}=\widehat{b}_{\ell}^{t}(\mathrm{t})\left(\Delta-2 \Delta_{\phi}-\ell\right)+O\left(\left(\Delta-2 \Delta_{\phi}-\ell\right)^{2}\right) .
\end{aligned}
$$

Crucially, it is the combination $\widehat{b}_{\ell}^{s}+\widehat{b}_{\ell}^{t}=\widehat{b}_{\ell}$ that benefits from the symmetry of eq. (D.8), and not the individual $s$ - or $t$-channel terms. Moreover, the symmetry that kills even spin, eq. (D.7), was already present at the level of the $\widehat{a}_{\ell}^{s}=\widehat{a}_{\ell}$ coefficient which is entirely captured by the $s$-channel Mack polynomials.

For mixed correlators, the prominent barrier to constructing a kernel $f_{\ell \mid m n}(\mathrm{t})$ is the lack of symmetry that could relate odd (even) spins $\ell$. To see this, let us explicitely evaluate the $\widehat{B}_{\mathbf{k} ; v \mid m n}$ functional for the $\langle A B A B\rangle$ correlator with $(m n)=(34)$ and $\mathbf{k}=(1,1,0,0)$ for which the analytic double-twist functional expansion is the same as for $\widehat{B}_{2 ; \mathrm{t}}$ for equal operators, but evaluated at $\Delta_{A}+\Delta_{B}+\ell$ instead. Doing so, we find

$$
\begin{aligned}
\widehat{a}_{\ell \mid 34}^{\Delta_{A}, \Delta_{B}, \Delta_{A}, \Delta_{B}}= & { }_{3} F_{2}\left(\left\{-\ell,-1+\ell+\Delta_{A}+\Delta_{B}, \frac{\Delta_{A}+\Delta_{B}-t}{2}\right\} ;\left\{\Delta_{A}, \Delta_{B}\right\} ; 1\right) \\
& \times(-1)^{\ell / 2} \frac{\Gamma\left(\ell+\Delta_{A}\right) \Gamma\left(\ell+\Delta_{B}\right)}{\Gamma\left(\Delta_{A}\right) \Gamma\left(\ell+\Delta_{B}\right) \Gamma(\ell+1)} .
\end{aligned}
$$

In contrast to the equal operator case, this hypergeometric function lacks any symmetry with respect to the Mandelstam variable $t$ which would allow us to write a variation of eq. (D.7). One could rewrite $\widehat{b}_{\ell}^{s, t}$ in terms of $\widehat{a}_{\ell}^{s, t}$ similar to eq. (4.56) of [30], but the lack of symmetry in Mandelstam $t$ reflects the inability for these Mack polynomials to diagonalize the OPE in both channels for all spin. It may be possible to derive projection operators for even and odd spins separately for the $\langle A B A B\rangle$ correlator, but we leave such investigations for future work. 


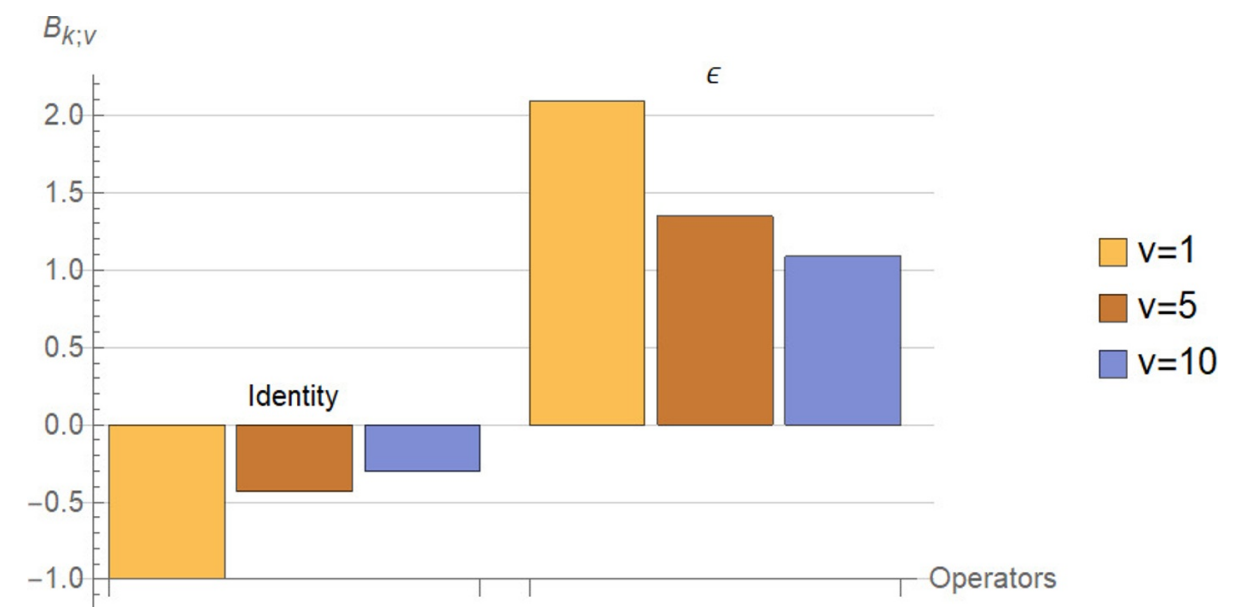

Figure 13. Contribution of certain operators to the sum rule of $\langle\sigma \sigma \sigma \sigma\rangle$ with the subtraction scheme $\left(k_{12}, k_{23}, k_{34}, k_{14}\right)=(1,1,0,0)$. Contribution of other operators are of order $O\left(10^{-2}\right)$ and less. The sum of all operators lead to $0.998,0.999$ and 0.999 for the three values of $v$ in the plot respectively.

\begin{tabular}{|c|cccc|}
\hline & $v=1$ & $v=5$ & $v=10$ & $v=100$ \\
\hline$\sum_{\left\{\mathcal{O}_{i}\right\} \in N_{3}} f_{\sigma \sigma \mathcal{O}_{i}}^{2} B_{\mathbf{k} ; v \mid 34}$ & 1.093 & 0.915 & 0.784 & 0.405 \\
\hline$\sum_{\left\{\mathcal{O}_{i}\right\} \in N_{\infty}} f_{\sigma \sigma \mathcal{O}_{i}}^{2} B_{\mathbf{k} ; v \mid 34}$ & & & & \\
\hline
\end{tabular}

Table 13. $N_{3}=\left\{1, \epsilon, T_{\mu \nu}\right\}$ and $N_{\infty}$ denote the set of all possible exchanged operators.
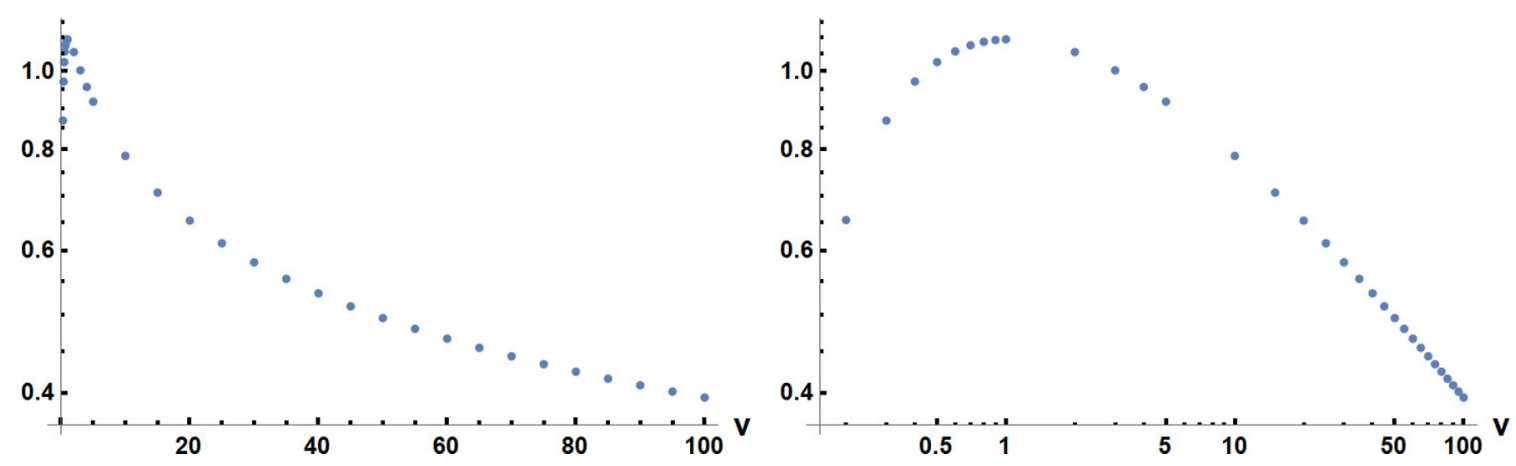

Figure 14. Ratio of the truncated sum over operators belonging to $N_{3}$ over the contribution of all operators $N_{\infty}$. The right panel is a log-log plot of the same data to highlight the small $v$ limit.

\section{E Varying $v$ for the $\langle\sigma \sigma \sigma \sigma\rangle$ sum rule}

We consider the $\langle\sigma \sigma \sigma \sigma\rangle$ correlator discussed in section 5.1. Figure 13 shows that the contribution of the most dominant operators diminish as we increase $v$. This is supported by table 13 which shows the contribution of the sum rule for the subset of operators $N_{3}=\left\{\mathbf{1}, \epsilon, T_{\mu \nu}\right\}$, while figure 14 shows how much these operators saturate the sum rule as we vary $v$. Increasing $v$ translates into integrating a smaller subset of the lightcone. As we shrink the integration domain, the contribution of all operators vanishes as a power-law. 
Open Access. This article is distributed under the terms of the Creative Commons Attribution License (CC-BY 4.0), which permits any use, distribution and reproduction in any medium, provided the original author(s) and source are credited.

\section{References}

[1] R. Rattazzi, V.S. Rychkov, E. Tonni and A. Vichi, Bounding scalar operator dimensions in 4D CFT, JHEP 12 (2008) 031 [arXiv:0807.0004] [InSPIRE].

[2] D. Poland, S. Rychkov and A. Vichi, The conformal bootstrap: theory, numerical techniques, and applications, Rev. Mod. Phys. 91 (2019) 015002 [arXiv: 1805.04405] [inSPIRE].

[3] P. Kravchuk, J. Qiao and S. Rychkov, Distributions in CFT. Part I. Cross-ratio space, JHEP 05 (2020) 137 [arXiv:2001.08778] [inSPIRE].

[4] P. Kravchuk, J. Qiao and S. Rychkov, Distributions in CFT. Part II. Minkowski space, JHEP 08 (2021) 094 [arXiv:2104.02090] [INSPIRE].

[5] S. El-Showk, M.F. Paulos, D. Poland, S. Rychkov, D. Simmons-Duffin and A. Vichi, Solving the 3D Ising model with the conformal bootstrap, Phys. Rev. D 86 (2012) 025022 [arXiv: 1203.6064] [INSPIRE].

[6] S. El-Showk, M.F. Paulos, D. Poland, S. Rychkov, D. Simmons-Duffin and A. Vichi, Solving the $3 d$ Ising model with the conformal bootstrap II. c-minimization and precise critical exponents, J. Stat. Phys. 157 (2014) 869 [arXiv:1403.4545] [INSPIRE].

[7] F. Kos, D. Poland and D. Simmons-Duffin, Bootstrapping mixed correlators in the 3D Ising model, JHEP 11 (2014) 109 [arXiv:1406.4858] [INSPIRE].

[8] S. Caron-Huot, Y. Gobeil and Z. Zahraee, The leading trajectory in the $2+1 D$ Ising CFT, arXiv: 2007.11647 [INSPIRE].

[9] F. Kos, D. Poland and D. Simmons-Duffin, Bootstrapping the $O(N)$ vector models, JHEP 06 (2014) 091 [arXiv:1307.6856] [INSPIRE].

[10] F. Kos, D. Poland, D. Simmons-Duffin and A. Vichi, Precision islands in the Ising and $O(N)$ models, JHEP 08 (2016) 036 [arXiv:1603.04436] [INSPIRE].

[11] S.M. Chester et al., Carving out OPE space and precise $O(2)$ model critical exponents, JHEP 06 (2020) 142 [arXiv: 1912.03324] [INSPIRE].

[12] I. Heemskerk, J. Penedones, J. Polchinski and J. Sully, Holography from conformal field theory, JHEP 10 (2009) 079 [arXiv: 0907.0151] [InSPIRE].

[13] J. Penedones, Writing CFT correlation functions as AdS scattering amplitudes, JHEP 03 (2011) 025 [arXiv:1011.1485] [INSPIRE].

[14] L. Rastelli and X. Zhou, Mellin amplitudes for $A d S_{5} \times S^{5}$, Phys. Rev. Lett. 118 (2017) 091602 [arXiv: 1608.06624] [INSPIRE].

[15] L.F. Alday and S. Caron-Huot, Gravitational S-matrix from CFT dispersion relations, JHEP 12 (2018) 017 [arXiv:1711.02031] [INSPIRE].

[16] S. Caron-Huot and A.-K. Trinh, All tree-level correlators in $A d S_{5} \times S_{5}$ supergravity: hidden ten-dimensional conformal symmetry, JHEP 01 (2019) 196 [arXiv: 1809.09173] [INSPIRE].

[17] V. Gonçalves, R. Pereira and X. Zhou, 20' five-point function from $A d S_{5} \times S^{5}$ supergravity, JHEP 10 (2019) 247 [arXiv:1906.05305] [INSPIRE]. 
[18] S. Kundu, Swampland conditions for higher derivative couplings from CFT, JHEP 01 (2022) 176 [arXiv:2104.11238] [INSPIRE].

[19] A. Kaviraj, K. Sen and A. Sinha, Analytic bootstrap at large spin, JHEP 11 (2015) 083 [arXiv: 1502.01437] [INSPIRE].

[20] D. Simmons-Duffin, The lightcone bootstrap and the spectrum of the 3d Ising CFT, JHEP 03 (2017) 086 [arXiv: 1612.08471] [INSPIRE].

[21] D. Mazac, Analytic bounds and emergence of $A d S_{2}$ physics from the conformal bootstrap, JHEP 04 (2017) 146 [arXiv:1611.10060] [INSPIRE].

[22] D. Mazac and M.F. Paulos, The analytic functional bootstrap. Part I. $1 D$ CFTs and 2D S-matrices, JHEP 02 (2019) 162 [arXiv:1803.10233] [INSPIRE].

[23] D. Mazac and M.F. Paulos, The analytic functional bootstrap. Part II. Natural bases for the crossing equation, JHEP 02 (2019) 163 [arXiv:1811.10646] [INSPIRE].

[24] D. Mazáč, A crossing-symmetric OPE inversion formula, JHEP 06 (2019) 082 [arXiv: 1812.02254] [INSPIRE].

[25] M.F. Paulos, Analytic functional bootstrap for CFTs in d > 1, JHEP 04 (2020) 093 [arXiv: 1910.08563] [INSPIRE].

[26] D. Mazáč, L. Rastelli and X. Zhou, A basis of analytic functionals for CFTs in general dimension, JHEP 08 (2021) 140 [arXiv:1910.12855] [INSPIRE].

[27] M.F. Paulos, Dispersion relations and exact bounds on CFT correlators, JHEP 08 (2021) 166 [arXiv: 2012.10454] [INSPIRE].

[28] A. Kaviraj, Crossing antisymmetric Polyakov blocks + dispersion relation, JHEP 01 (2022) 005 [arXiv: 2109.02658] [INSPIRE].

[29] D. Carmi and S. Caron-Huot, A conformal dispersion relation: correlations from absorption, JHEP 09 (2020) 009 [arXiv: 1910.12123] [INSPIRE].

[30] S. Caron-Huot, D. Mazac, L. Rastelli and D. Simmons-Duffin, Dispersive CFT sum rules, JHEP 05 (2021) 243 [arXiv:2008.04931] [INSPIRE].

[31] S. Caron-Huot, D. Mazac, L. Rastelli and D. Simmons-Duffin, AdS bulk locality from sharp CFT bounds, JHEP 11 (2021) 164 [arXiv:2106.10274] [inSPIRE].

[32] J. Penedones, J.A. Silva and A. Zhiboedov, Nonperturbative Mellin amplitudes: existence, properties, applications, JHEP 08 (2020) 031 [arXiv: 1912.11100] [INSPIRE].

[33] D. Carmi, J. Penedones, J.A. Silva and A. Zhiboedov, Applications of dispersive sum rules:

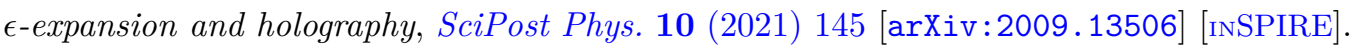

[34] R. Gopakumar, A. Sinha and A. Zahed, Crossing symmetric dispersion relations for Mellin amplitudes, Phys. Rev. Lett. 126 (2021) 211602 [arXiv:2101.09017] [INSPIRE].

[35] D. Meltzer, Dispersion formulas in QFTs, CFTs, and holography, JHEP 05 (2021) 098 [arXiv: 2103.15839] [INSPIRE].

[36] S. Caron-Huot and V. Van Duong, Extremal effective field theories, JHEP 05 (2021) 280 [arXiv : 2011.02957] [INSPIRE].

[37] S. Caron-Huot, D. Mazac, L. Rastelli and D. Simmons-Duffin, Sharp boundaries for the swampland, JHEP 07 (2021) 110 [arXiv:2102.08951] [INSPIRE]. 
[38] G. Mack, D-independent representation of conformal field theories in $D$ dimensions via transformation to auxiliary dual resonance models. Scalar amplitudes, arXiv:0907.2407 [INSPIRE].

[39] J. Penedones, TASI lectures on AdS/CFT, in Theoretical Advanced Study Institute in Elementary Particle Physics: new frontiers in fields and strings, World Scientific, Singapore (2017), pg. 75 [arXiv: 1608.04948] [INSPIRE].

[40] R. Gopakumar, A. Kaviraj, K. Sen and A. Sinha, Conformal bootstrap in Mellin space, Phys. Rev. Lett. 118 (2017) 081601 [arXiv:1609.00572] [INSPIRE].

[41] R. Gopakumar, A. Kaviraj, K. Sen and A. Sinha, A Mellin space approach to the conformal bootstrap, JHEP 05 (2017) 027 [arXiv: 1611.08407] [INSPIRE].

[42] R. Gopakumar and A. Sinha, On the Polyakov-Mellin bootstrap, JHEP 12 (2018) 040 [arXiv: 1809.10975] [INSPIRE].

[43] P. Haldar and A. Sinha, Froissart bound for/from CFT Mellin amplitudes, SciPost Phys. 8 (2020) 095 [arXiv: 1911.05974] [INSPIRE].

[44] S. Caron-Huot, Analyticity in spin in conformal theories, JHEP 09 (2017) 078 [arXiv: 1703.00278] [INSPIRE].

[45] M. Kologlu, P. Kravchuk, D. Simmons-Duffin and A. Zhiboedov, Shocks, superconvergence, and a stringy equivalence principle, JHEP 11 (2020) 096 [arXiv:1904.05905] [INSPIRE].

[46] M. Hogervorst and S. Rychkov, Radial coordinates for conformal blocks, Phys. Rev. D 87 (2013) 106004 [arXiv:1303.1111] [inSPIRE].

[47] M. Reehorst, S. Rychkov, D. Simmons-Duffin, B. Sirois, N. Su and B. van Rees, Navigator function for the conformal bootstrap, SciPost Phys. 11 (2021) 072 [arXiv:2104.09518] [INSPIRE].

[48] M. Reehorst, Rigorous bounds on irrelevant operators in the $3 d$ Ising model CFT, arXiv:2111.12093 [INSPIRE].

[49] R. Karlsson, M. Kulaxizi, A. Parnachev and P. Tadić, Black holes and conformal Regge bootstrap, JHEP 10 (2019) 046 [arXiv: 1904.00060] [INSPIRE].

[50] Y.-Z. Li, Heavy-light bootstrap from Lorentzian inversion formula, JHEP 07 (2020) 046 [arXiv: 1910.06357] [INSPIRE].

[51] Y.-Z. Li and H.-Y. Zhang, More on heavy-light bootstrap up to double-stress-tensor, JHEP 10 (2020) 055 [arXiv : 2004.04758] [INSPIRE].

[52] M.S. Costa, V. Goncalves and J. Penedones, Conformal Regge theory, JHEP 12 (2012) 091 [arXiv:1209.4355] [INSPIRE]. 